\title{
Differential calculus and connections on a quantum plane at a cubic root of unity
}

\author{
R. Coquereaux ${ }^{1 *}$, A. O. García ${ }^{2 \dagger}$, R. Trinchero ${ }^{2 \ddagger}$ \\ 1 Centre de Physique Théorique - CNRS - Luminy, Case 907 \\ F-13288 Marseille Cedex 9 - France \\ 2 Instituto Balseiro and Centro Atómico Bariloche \\ CC 439 - 8400 - Bariloche - Río Negro - Argentina
}

\begin{abstract}
We consider the algebra of $N \times N$ matrices as a reduced quantum plane on which a finitedimensional quantum group $\mathcal{H}$ acts. This quantum group is a quotient of $U_{q}(s l(2, \mathbb{C})), q$ being an $N$-th root of unity. Most of the time we shall take $N=3$; in that case $\operatorname{dim}(\mathcal{H})=27$. We recall the properties of this action and introduce a differential calculus for this algebra: it is a quotient of the Wess-Zumino complex. The quantum group $\mathcal{H}$ also acts on the corresponding differential algebra and we study its decomposition in terms of the representation theory of $\mathcal{H}$. We also investigate the properties of connections, in the sense of non commutative geometry, that are taken as 1-forms belonging to this differential algebra. By tensoring this differential calculus with usual forms over space-time, one can construct generalized connections with covariance properties with respect to the usual Lorentz group and with respect to a finitedimensional quantum group.
\end{abstract}

PACS: $02.90 .+\mathrm{p}, 11.30 .-\mathrm{j}$

MSC: 16W30, 81R50

Keywords: quantum groups, differential calculus, gauge theories, non commutative geometry.

Anonymous ftp or gopher: cpt.univ-mrs.fr

math-ph/9807012

CPT-98/P.3632

IT-CNEA-CAB/2906098

\footnotetext{
* Email: coque@cpt.univ-mrs.fr

$\dagger$ Email: ariel@cab.cnea.edu.ar

$\ddagger$ Email: trincher@cab.cnea.edu.ar
} 


\section{Introduction}

The algebra $\mathcal{M}$ of $N \times N$ complex matrices can be considered as a finite quantum space (indeed, a finite-dimensional vector space, as will be used in what follows). As such, it is a representation and corepresentation space for finite-dimensional (dual) quantum groups. If some physical problem involves such an algebra, it could therefore be tempting to think that this system has a (kind of) quantum symmetry. The role of fundamental object of the symmetry would now be played by a Hopf algebra, instead of a usual Lie group. In the case at hand the relevant quantum groups are particularly interesting, in view of the fact that, unlike the generic- $q$ case, they have a non-trivial radical and their representation theory involves indecomposable non-irreducible representations. Being finite-dimensional, they are also much simpler to analyse explicitly. Moreover, our knowledge about them is still much less complete than that of the generic- $q$ case.

Although our paper discusses several topics that can already be found in the literature (but we incorporate them to improve the reading, to set notations and for completeness' sake) we develop several aspects that, up to our knowledge, cannot be found elsewhere. Mainly, these include the construction of the (finite-dimensional) differential algebra over $\mathcal{M}$, the decomposition of this algebra and of its differential algebra as representations of the quantum group that acts on them, and the definition of a scalar product on $\mathcal{M}$ that is invariant under the quantum group action.

The algebra $\mathcal{M}$ can indeed be generated by two Heisenberg generators $x$ and $y$ satisfying the commutation relation $x y=q y x$, where $q$ is an $N$-root of unity. These basic facts are recalled in Section 2. As a consequence, there is on this algebra a coaction of a quotient $\mathcal{F}$ of the quantum group $F$ un $\left(S L_{q}(2, \mathbb{C})\right.$ ); this is reminded in Section 3. Consequently, the dual $\mathcal{H}$ of $\mathcal{F}$, also a finitedimensional quantum group, acts on the algebra of $N \times N$ matrices. Explicit formulae for the pairing between $\mathcal{H}$ and $\mathcal{F}$ and for the action of $\mathcal{H}$ on $\mathcal{M}$ have been given by [1, 2]. The structure of $\mathcal{H}$ had been studied before by [3] and its representation theory had been given by [4. In Section 4 we recall these duality properties and examine this action from the point of view of the reducible indecomposable representations of the quantum group $\mathcal{H}$, which is not a semisimple algebra. The unitary group of the semi-simple part of $\mathcal{H}$ turns out to be isomorphic with $U(3) \times U(2) \times U(1)$; for this reason, this Hopf algebra was conjectured (see [12]) to encode a quantum group of "hidden symmetries" in the standard model of electroweak interactions. However, the task of implementing this observation at the level of the lagrangian describing the theory has not been completed, and it seems that this idea would require some non trivial modifications of the model itself; nevertheless this remark provided one of the motivations for the present work.

In Section 5 we introduce a (unique) real structure on $\mathcal{M}, \mathcal{F}$ and $\mathcal{H}$ and construct a compatible hermitian scalar product on the space of matrices (the star operation on $\mathcal{M}$ does not coincide with the usual hermitian conjugacy on matrices). Next, in Section 5 we introduce the Manindual of our reduced quantum plane, and show how $\mathcal{F}$ coacts and $\mathcal{H}$ acts on it. In Section 7 we define differential forms on the algebra $\mathcal{M}$ and study their properties. The differential algebra $\Omega_{W Z}(\mathcal{M})$ that we introduce is a quotient of the Wess-Zumino complex [6] constructed originally for the 2-dimensional quantum plane. When $q$ is a third root of unity, $\mathcal{M}$ is the algebra of $3 \times 3$ matrices and $\mathcal{H}\left(\right.$ or $\mathcal{F}$ ) are of dimension 27. The differential algebra $\Omega_{W Z}(\mathcal{M})$ is of dimension 36. Since $\mathcal{H}$ acts on $\mathcal{M}$ and on its Manin dual, it also acts on $\Omega_{W Z}(\mathcal{M})$ and we study this algebra in terms of the representation theory of $\mathcal{H}$. Given an associative algebra - not necessarily commutative - one can define connections and covariant derivatives, for any choice of a differential calculus over the algebra of interest. The definition and properties of such connections are studied in Section 8; we also consider, as a particular case, connections that are hermitian for the star operation introduced before. In Section 9, we show how to couple these differential forms to usual space-time by constructing a differential algebra equal to the tensor product of $\Omega_{W Z}(\mathcal{M})$ with the usual differential forms (antisymmetric tensors on space-time). Generalized connections can then be defined. They incorporate a usual one-form valued in the space of $3 \times 3$ matrices and two matrix-valued scalar fields. These connections transform covariantly under a simultaneous action of the usual Lorentz group and the finite-dimensional quantum group $\mathcal{H}$. It would certainly be interesting to build a classical Lagrangian field theory along these lines, but this involves some deeper problems that are mentioned in the concluding section.

The paper ends with a number of short appendices. In them we first describe a set of $3 \times 3$ 
generalized Gell-Mann matrices with entries in the quantum group $\mathcal{F}$. Then we give the structure of the principal indecomposable modules and the most general covariant metrics on the representation spaces of the algebra $\mathcal{H}$, we study the space of differential operators on $\mathcal{M}$ and finally write down the universal $R$-matrix of $\mathcal{H}$. 


\section{The space $\mathcal{M}$ of $3 \times 3$ complex matrices as a reduced quan- tum plane}

\subsection{From elementary $N \times N$ matrices to the $x, y$ generators}

It has been known for a long time $[7$ that the algebra of $N \times N$ matrices can be generated by two elements $x$ and $y$ with the relations

$$
x y=q y x, \quad x^{N}=y^{N}=\mathbb{1},
$$

where $q$ denotes an $N$-th root of unity $(q \neq 1)$ and $\mathbb{1}$ is the unit matrix.

Let us make explicit, in the particular case $N=3$, this well known (but sometimes forgotten...) property.

Let $q$ be a cubic root of unity $(q \neq 1)$ and take

$$
x=\left(\begin{array}{ccc}
1 & 0 & 0 \\
0 & q^{-1} & 0 \\
0 & 0 & q^{-2}
\end{array}\right) \quad y=\left(\begin{array}{ccc}
0 & 1 & 0 \\
0 & 0 & 1 \\
1 & 0 & 0
\end{array}\right) .
$$

It is then easy to check that the above relations between $x$ and $y$ are indeed satisfied (use $q^{-1}=q^{2}$ ), and that they generate the whole algebra. Many formulae that we shall write in the following can be easily generalized when $N$ is an arbitrary integer, but we shall stick to the case $N=3$.

Any $3 \times 3$ matrix can obviously be expanded on the base made of the nine elementary matrices $E_{i j}$ (such a matrix has a single non-zero entry 1 in position $(i, j)$ and is filled with zeros elsewhere). One can express the elementary matrices themselves in terms of $x$ and $y$. Calculations are straightforward, and give

$$
\begin{array}{ll}
E_{11}=\left(\mathbb{1}+x+x^{2}\right) / 3 & E_{12}=\left(y+x y+x^{2} y\right) / 3 \\
E_{13}=\left(y^{2}+x y^{2}+x^{2} y^{2}\right) / 3 & E_{21}=\left(y^{2}+q x y^{2}+q^{2} x^{2} y^{2}\right) / 3 \\
E_{22}=\left(\mathbb{1}+q x+q^{2} x^{2}\right) / 3 & E_{23}=\left(y+q x y+q^{2} x^{2} y\right) / 3 \\
E_{31}=\left(y+q^{2} x y+q x^{2} y\right) / 3 & E_{32}=\left(y^{2}+q^{2} x y^{2}+q x^{2} y^{2}\right) / 3 \\
E_{33}=\left(\mathbb{1}+q^{2} x+q x^{2}\right) / 3 &
\end{array}
$$

The unit matrix (1) itself can be written in terms of the generators $x$ and $y$ (since $x^{3}=y^{3}=\mathbb{1}$ ). Therefore, one can also express the usual Gell-Mann matrices that generate $\operatorname{Lie}(S U(3))$ in terms of the generators $x$ and $y$. These expressions are shown in Appendix A.

Warning: the set of $3 \times 3$ matrices is endowed with a usual star operation (that we denote $\dagger$ ): hermitian conjugacy. It is clear that $x$ and $y$ are unitary elements (with respect to $\dagger$ ): $x^{\dagger}=x^{-1}=$ $x^{2}$ and $y^{\dagger}=y^{-1}=y^{2}$. As a matter of fact, this star operation does not have good properties with respect to a quantum group action that we shall introduce later. We shall return to this important problem in a forthcoming section.

\section{2 $\mathcal{M}=M_{3}(\mathbb{C})$ as a reduced quantum plane}

The associative algebra generated, over the complex numbers, by $x$ and $y$ with the single (quadratic) relation $y x=q^{-1} x y$ is known as "the algebra of polynomials over the quantum plane" and is often denoted by Fun $\left(\mathbb{C}^{2}\right)$ or by $\mathbb{C}_{q}[x, y]$. We shall just call it $\mathbb{C}_{q}$. When $q=1$, this algebra is commutative and can be considered as the algebra of polynomials $\mathbb{C}[x, y]$ over the usual plane, $x$ and $y$ being the two coordinate functions. The dimension of $\mathbb{C}_{q}$ - as a vector space - is infinite, since powers of the generators do not satisfy any particular new relation. On the contrary, in the algebra $M_{3}(\mathbb{C})$ of $3 \times 3$ matrices over complex numbers, the generators $x$ and $y$, on top of the above quadratic relation, satisfy also the cubic relations $x^{3}=\mathbb{1}$ and $y^{3}=\mathbb{1}$. The dimension is then clearly equal to 9 , as it should, since one can choose the following base of generators: $\left\{\mathbb{1}, x, y, x^{2}, y^{2}, x y, x^{2} y, x y^{2}, x^{2} y^{2}\right\}$. One can therefore consider $M_{3}(\mathbb{C})$ as the quotient of the associative algebra $\hat{\mathbb{C}}_{q}$, when $q^{3}=1$, by the bilateral ideal generated by the relations $x^{3}-\mathbb{1}=0$ and $y^{3}-\mathbb{1}=0$. Here $\hat{\mathbb{C}}_{q}$ denotes the unital extension of $\mathbb{C}_{q}$ (the former is obtained by adding a unit, namely $\mathbb{1}$ to the later). For this reason we can consider the space of $3 \times 3$ matrices over $\mathbb{C}$ as a reduced quantum plane.

Warning: $\mathcal{M} \doteq M_{3}(\mathbb{C})$ is not a quantum group. 


\section{The finite-dimensional quantum group $\mathcal{F}$}

\subsection{Construction of $\mathcal{F}$ as an algebra}

Let us start with a classical analogy and call $x$ and $y$ the coordinate functions on the plane. One can make a linear change of coordinates and call $x^{\prime}$ and $y^{\prime}$ the new coordinate functions:

$$
\left(\begin{array}{l}
x^{\prime} \\
y^{\prime}
\end{array}\right)=\left(\begin{array}{ll}
a & b \\
c & d
\end{array}\right) \otimes\left(\begin{array}{l}
x \\
y
\end{array}\right)
$$

We can assume the transformation to be unimodular $(a d-b c=1)$. Rather than considering $x$ and $y$ as numbers, we think of them as coordinate functions. In the same way, we do not take the matrix elements $a, b, c$ and $d$ as numbers but as functions on the group of coordinate transformations: when $g$ denotes such a transformation then $a(g), b(g), c(g)$ and $d(g)$ are numbers, namely, the matrix elements of $g$. This change in the perspective explains why we write a tensor product sign in the previous formula... evaluation on points of the group and of the space gives the transformed coordinate functions.

One could also introduce line vectors, with coordinate functions $\tilde{x}, \tilde{y}$. The same change of coordinates would read

$$
\left(\begin{array}{ll}
\tilde{x} & \tilde{y}
\end{array}\right)=\left(\begin{array}{ll}
x & y
\end{array}\right) \otimes\left(\begin{array}{ll}
a & b \\
c & d
\end{array}\right) .
$$

From now on, we shall no longer assume that symbols $x$ and $y$ commute but that they should satisfy the relations discussed in the previous section, namely $x y=q y x, x^{3}=y^{3}=\mathbb{1}$. Symbols $x$ and $y$ can therefore be represented by the $3 \times 3$ matrices already given. As before, $\mathcal{M}$ shall denote the algebra generated by $x$ and $y$.

One then introduces non-commuting symbols $a, b, c$ and $d$ and imposes that quantities $x^{\prime}, y^{\prime}$ (and $\tilde{x}, \tilde{y}$ ) obtained by the previous matrix equalities should satisfy the same relations as $x$ and $y$. We call $\mathcal{F}^{\prime}$ the algebra generated by the elements $a, b, c$ and $d$. The product in $\mathcal{F}^{\prime} \otimes \mathcal{A}$ is defined by $(f \otimes z)(g \otimes w) \doteq f g \otimes z w$, in other words, $a, b, c, d$ commute with $x, y$. We have a similar definition for the multiplication in $\mathcal{A} \otimes \mathcal{F}^{\prime}$. The two constraints $x^{\prime} y^{\prime}=q y^{\prime} x^{\prime}$ and $\tilde{x} \tilde{y}=q \tilde{y} \tilde{x}$ lead to the six quadratic relations [8]

$$
\begin{array}{ll}
a c=q c a & b d=q d b \\
a b=q b a & c d=q d c \\
b c=c b & a d-d a=\left(q-q^{-1}\right) b c
\end{array}
$$

The algebra generated by $a, b, c$ and $d$ (take products and sums) together with the six above relations is usually denoted $F u n\left(G L_{q}(2, \mathbb{C})\right)$ and is the algebra of would-be functions over the quantum group $G L_{q}(2, \mathbb{C})$. Calling such elements "functions" is, of course, a misnomer, since they are not valued in any field of numbers and... do not commute.

The element $\mathcal{D} \doteq d a-q^{-1} b c=a d-q b c$ is central (it commutes with all the elements of Fun $\left.\left(G L_{q}(2)\right)\right)$; it is called the $q$-determinant and we set it equal to $\mathbb{1}$. Adding this extra relation defines the algebra $\operatorname{Fun}\left(S L_{q}(2, \mathbb{C})\right)$.

Now, we should remember that $x^{3}=y^{3}=\mathbb{1}$. Imposing $x^{\prime 3}=y^{\prime 3}=\mathbb{1}\left(\right.$ and also $\left.\tilde{x}^{3}=\tilde{y}^{3}=\mathbb{1}\right)$ implies again new relations.

For instance,

$$
\begin{aligned}
x^{\prime 3}= & (a \otimes x+b \otimes y)^{3} \\
= & a^{3} \otimes x^{3}+a^{2} b \otimes x^{2} y+a b a \otimes x y x+b a^{2} \otimes y x^{2}+ \\
& a b^{2} \otimes x y^{2}+b a b \otimes y x y+b^{2} a \otimes y^{2} x+b^{3} \otimes y^{3} \\
= & a^{3} \otimes x^{3}+\left(1+q+q^{2}\right) a^{2} b \otimes x^{2} y+\left(1+q+q^{2}\right) a b^{2} \otimes x y^{2}+b^{3} \otimes y^{3} \\
= & a^{3} \otimes x^{3}+b^{3} \otimes y^{3}
\end{aligned}
$$

where we used $1+q+q^{2}=0$ since $q$ is a third root of unity.

\footnotetext{
${ }^{1}$ We will use 1 to denote the unit element of $\mathcal{M}, \mathcal{F}$ and the - to be defined- quantum group $\mathcal{H}$, indistinctly. Which one this symbol refers to, should be easily understood from context. In case this were not obvious we will use $\mathbf{1}_{\mathcal{M}}, \mathbf{1}_{\mathcal{F}}, \mathbf{1}_{\mathcal{H}}$.
} 
This, together with the analogous constraints on $y^{\prime}, \tilde{x}, \tilde{y}$, imply:

$$
\begin{array}{ll}
a^{3}=\mathbb{1}, & b^{3}=0, \\
c^{3}=0, & d^{3}=\mathbb{1} .
\end{array}
$$

Imposing these cubic relations on $F u n\left(S L_{q}(2, \mathbb{C})\right)$ defines a new algebra that we denote with $\mathcal{F}$. We call it the reduced quantum unimodular group associated with a cubic root of unity. Actually one should better call it the algebra of would-be functions over the reduced quantum unimodular group $S L_{q}(2, \mathbb{C})$ but we shall shorten the terminology.

Since $a^{3}=\mathbb{1}$, multiplying the relation $a d=\mathbb{1}+q b c$ from the left by $a^{2}$ leads to

$$
d=a^{2}(\mathbb{1}+q b c)
$$

so $d$ is not needed and can be eliminated. The algebra $\mathcal{F}$ can therefore be linearly generated - as a vector space - by the elements $a^{\alpha} b^{\beta} c^{\gamma}$ where indices $\alpha, \beta, \gamma$ run in the set $\{0,1,2\}$. We see that $\mathcal{F}$ is a finite-dimensional associative algebra, whose dimension is

$$
\operatorname{dim}(\mathcal{F})=27 .
$$

Let us stress the fact that this very particular algebra $\mathcal{F}$ (which turns out to be a quantum group) emerges naturally in relation with the study of the algebra $\mathcal{M}$ of $3 \times 3$ matrices.

\section{2 $\mathcal{F}$ as a quantum group}

$\mathcal{F}$ is not only an algebra but also a quantum group, in other words, a Hopf algebra. This amounts to say that besides its algebra structure (a product), it has a coalgebra structure (a coproduct) and that the two structures are compatible (hence it is a bialgebra); moreover one can also define maps called antipode and counit obeying the appropriate axioms.

There are now several more or less elementary textbooks on the subject of quantum groups and the interested reader should refer to them for general properties. We shall simply give the definitions for the coproduct, antipode and counit, in the present case. The reader will easily show that all required properties are indeed satisfied. These three maps being algebra morphisms (or antimorphisms) it is actually enough to define them on the generators.

Coproduct: It is an algebra morphism $\Delta$ from the algebra $\mathcal{F}$ to the algebra $\mathcal{F} \otimes \mathcal{F}$, i.e., $\Delta(u v)=$ $\Delta u \Delta v$. It is given by $\Delta a=a \otimes a+b \otimes c, \Delta b=a \otimes b+b \otimes d, \Delta c=c \otimes a+d \otimes c, \Delta d=c \otimes b+d \otimes d$.

Antipode: It is a (linear 2 antimorphism $S$ from $\mathcal{F}$ to $\mathcal{F}$, i.e., $S(u v)=S v S u$. It is given by $S a=d, S b=-q^{-1} b, S c=-q c, S d=a$.

Counit: It is a morphism $\epsilon$ from $\mathcal{F}$ to the complex numbers $\mathbb{C}$ and is given by $\epsilon(a)=1, \epsilon(b)=0$, $\epsilon(c)=0, \epsilon(d)=1$.

Actually, one can give a rather short proof of the fact that $\mathcal{F}$ is a Hopf algebra. It is enough to prove that the two-sided ideal $\mathcal{I}$ defining the quotient $\mathcal{F}=\operatorname{Fun}\left(S L_{q}(2, \mathbb{C})\right) / \mathcal{I}$ is a Hopf ideal. $\mathcal{I}$ is the ideal generated by the relations $a^{3}-\mathbf{1}=0, d^{3}-\mathbf{1}=0, b^{3}=0$ and $c^{3}=0$. Being $\mathcal{I}$ a Hopf ideal means: $\Delta \mathcal{I} \subset \mathcal{I} \otimes \operatorname{Fun}\left(S L_{q}(2, \mathbb{C})\right) \oplus \operatorname{Fun}\left(S L_{q}(2, \mathbb{C})\right) \otimes \mathcal{I}, \epsilon(\mathcal{I})=0$ and $S(\mathcal{I}) \subset I$. This is easy to show. For instance, we see that

$$
\begin{aligned}
\Delta a^{3} & =(\Delta a)^{3}=(a \otimes a+b \otimes c)^{3} \\
& =a^{3} \otimes a^{3}+\left(1+q+q^{2}\right) a^{2} b \otimes a^{2} c+\left(1+q+q^{2}\right) a b^{2} \otimes a c^{2}+b^{3} \otimes c^{3} \\
& =a^{3} \otimes a^{3}+b^{3} \otimes c^{3}
\end{aligned}
$$

but $\Delta \mathbb{1}=\mathbb{1} \otimes \mathbb{1}$, so that

$$
2 \Delta\left(a^{3}-\mathbf{1}\right)=a^{3} \otimes\left(a^{3}-\mathbf{1}\right)+\left(a^{3}-\mathbf{1}\right) \otimes a^{3}+\left(a^{3}-\mathbb{1}\right) \otimes \mathbf{1}+\mathbb{1} \otimes\left(a^{3}-\mathbf{1}\right)+2 b^{3} \otimes c^{3}
$$

which is indeed in $\mathcal{I} \otimes F u n\left(S L_{q}(2, \mathbb{C})\right) \oplus F \operatorname{un}\left(S L_{q}(2, \mathbb{C})\right) \otimes \mathcal{I}$.

$\mathcal{F}$ is, by construction, an associative algebra. However, it is not semisimple. This can be seen easily from the faithful realization given in Appendix C. (this realization, in terms of matrices with entries in an algebra generated by two commuting symbols $\xi_{1}, \xi_{2}$ whose cube power vanishes is due to Ogievetsky, [9]). $\mathcal{F}$ is therefore not a matrix quantum group in the sense of Woronowicz [10]: whatever the involution and the scalar product we choose, it will never be a $C^{*}$-algebra.

\footnotetext{
${ }^{2}$ We stress that the antipode is a linear antimorphism, in contrast with the star operation that will be introduced in Section 1 which is an antilinear antimorphism.
} 


\section{$3.3 \mathcal{F}$ coacting on $\mathcal{M}$}

What is called coaction of $\mathcal{F}$ on $\mathcal{M}$ is precisely what was described, in simple terms, at the beginning of this section. Actually, we have two coactions: a left coaction and a right one. We have

$$
\begin{aligned}
x^{\prime} \doteq \Delta_{L} x & =a \otimes x+b \otimes y \\
y^{\prime} \doteq \Delta_{L} y & =c \otimes x+d \otimes y
\end{aligned}
$$

and

$$
\begin{aligned}
& \tilde{x} \doteq \Delta_{R} x=x \otimes a+y \otimes c \\
& \tilde{y} \doteq \Delta_{R} y=x \otimes b+y \otimes d
\end{aligned}
$$

Both $\Delta_{L}$ and $\Delta_{R}$ can be extended to arbitrary elements of $\mathcal{M}$ by imposing the property

$$
\Delta_{L, R}(z w)=\Delta_{L, R}(z) \Delta_{L, R}(w)
$$

for any $z, w \in \mathcal{M}$.

$\mathcal{M}$ itself, endowed with the two coactions $\Delta_{L}$ and $\Delta_{R}$ is not a quantum group but a left and right comodule, i.e., a corepresentation space of the quantum group $\mathcal{F}$. This means that the right coaction (for instance) maps $\mathcal{M} \mapsto \mathcal{M} \otimes \mathcal{F}$, in such a way that

$$
\left(\Delta_{R} \otimes i d\right) \Delta_{R}(z)=(i d \otimes \Delta) \Delta_{R}(z)
$$

and

$$
(i d \otimes \epsilon) \Delta_{R}(z)=z,
$$

for any $z \in \mathcal{M}$. Here one should not confuse $\Delta$ (the coproduct on the quantum group) with $\Delta_{R, L}$ (the $R, L$-coaction on $\mathcal{M}$ )! These conditions are indeed satisfied... the interested reader may check that.

Moreover, $\mathcal{M}$ is a left and right comodule algebra over $\mathcal{F}$. There are two extra axioms that have to be satisfied in order to have a comodule algebra structure, in particular a compatibility axiom between the coaction and the product in $\mathcal{M}$. These extra conditions read:

$$
\Delta_{L, R}(z w)=\Delta_{L, R}(z) \Delta_{L, R}(w)
$$

for $z, w$ any elements of the algebra. This is just the equation we have used to extend the coaction to the whole $\mathcal{M}$, thus is trivially satisfied. If the algebra also has a unit, the coaction should verify

$$
\Delta_{L, R}(\mathbb{1})=\mathbb{1}_{\otimes} .
$$

Using this coaction, and recalling that $\mathcal{M}$ is the algebra of $3 \times 3$ matrices, one can build a set of generalized Gell-Mann matrices with entries in the quantum group $\mathcal{F}$, as it is done in Appendix B.

Notice that although $\mathcal{F}$ coacts on $\mathcal{M}$, it does not act on it. This is exactly what happens in the usual (commutative) situation: the algebra of functions on the group of unimodular transformations of the plane coacts on the algebra of functions on the plane (there are indeed tensor product signs in the formula $x \rightarrow a \otimes x+b \otimes y$, even if it is sometimes convenient not to write them...). In the present situation, we have no "points" and it makes a priori no sense to speak about the action of a would-be group on would-be points. The coaction of $\mathcal{F}$ on $\mathcal{M}$ is however well defined and allows such a terminological abuse.

As a matter of fact, there is an algebra that acts on $\mathcal{M}$, it is not $\mathcal{F}$ but its dual, that we call $\mathcal{H}$. We shall return to this in the next section.

\footnotetext{
3 Taking into account this condition, we see that the equations

$$
\Delta_{R, L}(x y-q y x)=0
$$
}

are completely equivalent to the ones we used in Section 3.1 to obtain the relations (里) for the quantum group $\mathcal{F}$. Therefore they also imply (4). 


\section{The dual $\mathcal{H}$ of $\mathcal{F}$}

\subsection{Classical analogies}

One should think of $\mathcal{F}$ as an analogue of the space of functions $F u n(G)$ over a Lie group $G$ (in our case, $G$ itself does not exist) and $\mathcal{H}$ - that we shall introduce now- as a finite-dimensional analogue of the universal enveloping algebra of the Lie algebra of $G$. Another fruitful analogy is to take $\mathcal{F}$ as the analogue of the space of functions over a finite group $G$ and $\mathcal{H}$ as an analogue of the group algebra $\mathbb{C} G$.

As in the classical case, arbitrary elements $X$ of $\mathcal{H}$ can be understood in several ways:

- As distributions on $\mathcal{F}$ : we evaluate $X$ on the "function" $u \in \mathcal{F}$. The result is a number called $\langle X, u\rangle$.

- As invariant differential operators acting on the "functions" $u$. The result is another element of $\mathcal{F}$, that we call $X[u]$. Actually, as in the classical case, one can define two kinds of invariant operators: left-invariant ones (coming classically from the right action of a group on itself) and right-invariant ones (coming from the left action).

- As a differential operator acting on the space of "functions" $\mathcal{M}$ (classically functions on a manifold on which the group $G$ acts). Let $z$ be such a function. We call $X[z]$ the result. This is another element of $\mathcal{M}$.

- Abstractly, as an element of the associative algebra $\mathcal{H}$, i.e., classically, an element of the enveloping algebra $\mathcal{U}(\operatorname{Lie}(G))$.

The remaining part of this section is devoted to a short study of these various aspects.

\subsection{The finite-dimensional quantum group $\mathcal{H}$}

Being the dual of $\mathcal{F}$, it is clear that $\mathcal{H}$ is a vector space of dimension 27. It can be generated, as a complex algebra, by elements $X_{ \pm}, K$ and $K^{-1}$ obeying a number of relations. Multiplication and comultiplication in $\mathcal{H}$ can be obtained from the corresponding ones in its dual $\mathcal{F}$, but here we gather most of the relevant formulae abstractly defining $\mathcal{H}$ as a Hopf algebra, with generators $X_{ \pm}$ and $K$, without using its duality with $\mathcal{F}$.

\section{Product:}

$$
\begin{aligned}
K X_{ \pm} & =q^{ \pm 2} X_{ \pm} K \\
{\left[X_{+}, X_{-}\right] } & =\frac{1}{\left(q-q^{-1}\right)}\left(K-K^{-1}\right) \\
K^{3} & =\mathbb{1} \\
X_{+}^{3}=X_{-}^{3} & =0
\end{aligned}
$$

Coproduct: The comultiplication is an algebra morphism, i.e., $\Delta(X Y)=\Delta X \Delta Y$. It is given by

$$
\begin{aligned}
\Delta X_{+} & \doteq X_{+} \otimes \mathbb{1}+K \otimes X_{+} \\
\Delta X_{-} & \doteq X_{-} \otimes K^{-1}+\mathbb{1} \otimes X_{-} \\
\Delta K & \doteq K \otimes K \\
\Delta K^{-1} & \doteq K^{-1} \otimes K^{-1}
\end{aligned}
$$

Antipode: The antipode $S$ is an anti-automorphism, i.e., $S(X Y)=S Y S X$. It acts as follows: $S \mathbb{1}=\mathbb{1}, S K=K^{-1}, S K^{-1}=K, S X_{+}=-K^{-1} X_{+}, S X_{-}=-X_{-} K$. As usual, the square of the antipode is an automorphism (and it is a conjugacy given - up to multiplication by a central element- by $K^{-1}$, hence we can write $\left.S^{2} u=K^{-1} u K\right)$.

Counit: The counit $\epsilon$ is defined by $\epsilon \mathbb{1}=1, \epsilon K=1, \epsilon K^{-1}=1, \epsilon X_{+}=0, \epsilon X_{-}=0$.

The previous multiplication relations allow to order any monomial of the algebra freely generated by $X_{ \pm}$and $K$ as $X_{+}^{\alpha} K^{\beta} X_{-}^{\gamma}$. Moreover, as $X_{ \pm}^{3}=0$ and $K^{3}=\mathbb{1}$, it is evident that the $27=3^{3}$ elements $\left\{\left(X_{+}^{\alpha} K^{\beta} X_{-}^{\gamma}\right)\right\}_{\alpha, \beta, \gamma \in\{0,1,2\}}$ span $\mathcal{H}$ as a vector space over $\mathbb{C}$. 
Note that the element $X_{+} X_{-}-\left(q^{-1} K+q K^{-1}\right) / 3$ commutes with all elements of $\mathcal{H}$. Therefore its plays the role of a usual Casimir operator.

Remark: In [1] the authors study the pairing between a (reduced) universal enveloping algebra and the algebra of functions on quantum $S L(2, \mathbb{C})$, in the case $q^{N}=1$. However, they factorise the universal algebra over the relations $k^{6}=\mathbb{1}, I_{ \pm}^{3}=0$, rather than $K^{3}=\mathbf{1}, X_{ \pm}^{3}=0$. Their choice for generators $I_{ \pm}$and $k$ differs from our $X_{ \pm}$and $K$ (actually, $k=K^{1 / 2}, I_{+}=q X_{+} K$ and $I_{-}=q X_{-} K^{2}$ ). The obtained algebra $\tilde{\mathcal{H}}$ is twice as big as ours $\left(k^{3}\right.$ is a central element but is not equal to $\left.\mathbb{1}\right)$.

\section{3 $\mathcal{H}$ as the dual of $\mathcal{F}$ (distributions)}

Being $\mathcal{F}$ a quantum group (a Hopf algebra), its dual $\mathcal{H} \doteq \mathcal{F}^{*}$ is a quantum group as well. Let $u \in \mathcal{F}$ and $X \in \mathcal{H}$. We call $\langle X, u\rangle$ the evaluation of $X$ on $u$ (a complex number).

- Using the coproduct $\Delta$ in $\mathcal{F}$, one defines a product in $\mathcal{H}$ (this is the so-called convolution product of distributions and it is often denoted by $\star$, but we shall omit this symbol in the sequel):

$$
<X_{1} X_{2}, u>\doteq<X_{1} \otimes X_{2}, \Delta u>
$$

- Using the product in $\mathcal{F}$, one defines a coproduct (that we again denote $\Delta$ ) in $\mathcal{H}$ :

$$
<\Delta X, u_{1} \otimes u_{2}>\doteq<X, u_{1} u_{2}>
$$

- The interplay between unit and counit is described by the two relations: $\left\langle\mathbb{1}_{\mathcal{H}}, u\right\rangle=\epsilon_{\mathcal{F}}(u)$ and $\left\langle X, \mathbb{1}_{\mathcal{F}}\right\rangle=\epsilon_{\mathcal{H}}(X)$.

The two structures of algebra and coalgebra are clearly interchanged by duality.

$\mathcal{H}$ is the linear dual of $\mathcal{F}$. In other words, if $\mathcal{F}$ were a space of smooth (resp. continuous) functions over a compact space, $\mathcal{H}$ would be the corresponding space of distributions (resp. measures).

All possible pairings can be computed from those between the generators $K, X_{ \pm}$and $a, b, c$. They are given by:

$$
\begin{array}{cccc}
<K, a>=q & <K, b>=0 & <K, c>=0 & <K, d>=q^{2} \\
<X_{+}, a>=0 & <X_{+}, b>=1 & <X_{+}, c>=0 & <X_{+}, d>=0 \\
<X_{-}, a>=0 & <X_{-}, b>=0 & <X_{-}, c>=1 & <X_{-}, d>=0
\end{array}
$$

The fourth column of this table can be obtained from the first three (remember that $d=a^{2}(\mathbb{1}+q b c)$ ).

Remark: the previously given multiplication and comultiplication equalities for the generators of $\mathcal{H}$ (in particular the appearance of $q$ rather than its inverse) cannot be given arbitrarily; indeed they have to be compatible with those given for the dual, $\mathcal{F}$. In other words, once the duality formulae defining the generators $X_{ \pm}$have been chosen, one cannot choose independently "the $q$ of $\mathcal{F}$ " and "the $q$ of $\mathcal{H}$ ". Conversely, if we start with the formulae defining multiplication and comultiplication in $\mathcal{F}$ and $\mathcal{H}$, the pairing formulae are essentially unique.

To see this, let us suppose given, for instance, the comultiplication relations for $\mathcal{H}$ and determine the constraints for both the pairings and the multiplication in $\mathcal{H}$. Choose the basis $a^{i} b^{j} c^{k}$ in the vector space $\mathcal{F}$. At the same time we choose a basis $K^{i} X_{+}^{j} X_{-}^{k}$ in the dual vector space $\mathcal{F}$, with pairings

$$
\begin{array}{lc}
<K, a>=\alpha & <K, b>=<K, c>=0 \\
<X_{+}, b>=\beta & <X_{+}, a>=<X_{+}, c>=0 \\
<X_{-}, c>=\gamma & <X_{-}, a>=<X_{-}, b>=0
\end{array}
$$

etc. where $\alpha, \beta, \gamma$ are numbers that we are going to determine.

First of all, let us calculate $\left\langle X_{+}, a b\right\rangle$ and $\left\langle X_{+}, b a\right\rangle$. Known commutation relations in $\mathcal{F}$ (or comultiplication in $\mathcal{H}$ ) will imply that $\alpha=q$. Indeed,

$$
\begin{aligned}
& <X_{+}, a b>=<\Delta X_{+}, a \otimes b>=<X_{+} \otimes \mathbb{1}+K \otimes X_{+}, a \otimes b>=0+\alpha \beta \\
& <X_{+}, b a>=<\Delta X_{+}, b \otimes a>=<X_{+} \otimes \mathbb{1}+K \otimes X_{+}, b \otimes a>=\beta+0 .
\end{aligned}
$$

\footnotetext{
4 Some authors define $\left\langle\Delta X, u_{1} \otimes u_{2}\right\rangle \doteq<X, u_{2} u_{1}>$, in order to increase the correspondence with the classical case. This alternative definition corresponds to use our $\Delta^{o p}=\tau \circ \Delta$ as a coproduct, where $\tau$ produces a permutation of factors in the tensor product.
} 
But $a b=q b a$, therefore $\alpha=q$.

Then, we shall calculate $\left\langle K X_{+}, b\right\rangle$ and $\left\langle X_{+} K, b\right\rangle$. This will imply $K X_{+}=q^{2} X_{+} K$. To do that, we need to find $\langle K, d\rangle$. We know that $d=a^{2}(\mathbb{1}+q b c)$, therefore

$$
\begin{aligned}
<K, d>= & <\Delta K, a^{2} \otimes(\mathbf{1}+q b c)>=<K, a^{2}><K,(\mathbf{1}+q b c)> \\
= & <\Delta K, a \otimes a>(<K, \mathbf{1}>+q<K, b c>)=q^{2}(1+0)=q^{2}
\end{aligned}
$$

Now,

$$
\begin{aligned}
<K X_{+}, b>= & <K \otimes X_{+}, \Delta b>=<K \otimes X_{+}, a \otimes b+b \otimes d> \\
= & <K, a><X_{+}, b>+<K, b><X_{+}, d>=q \beta+0 .
\end{aligned}
$$

But

$$
\begin{aligned}
<X_{+} K, b>= & <X_{+} \otimes K, \Delta b>=<X_{+} \otimes K, a \otimes b+b \otimes d> \\
= & <X_{+}, a><K, b>+<X_{+}, b><K, d>=0+\beta q^{2}
\end{aligned}
$$

Therefore

$$
<X_{+} K, b>=q<K X_{+}, b>\quad \longrightarrow \quad K X_{+}=q^{2} X_{+} K
$$

Finally, there are no conditions on $\beta, \gamma$ and we can take $\beta=1$ and $\gamma=1$.

We conclude that the following conditions are compatible: $a b=q b a,<K, a>=q, X_{+} K=q^{2} X_{+} K$. Such considerations justify the defining relations given a priori, in Section 4.2 .

\subsection{Actions of $\mathcal{H}$}

One can define several actions of $\mathcal{H}$ on $\mathcal{H}$, of $\mathcal{H}$ on $\mathcal{F}$ and of $\mathcal{H}$ on $\mathcal{M}$. We briefly mention them in the present subsection.

\section{$\diamond \quad \mathcal{H}$ acting on $\mathcal{H}$}

There is a natural action of $\mathcal{H}$ on itself given by the multiplication, thus we can define:

- the right action $R[X] Y=Y X$,

- the right action $R^{\prime}[X] Y=S(X) Y$,

- the left action $L[X] Y=X Y$,

- the left action $L^{\prime}[X] Y=Y S(X)$.

However, none of these actions is compatible with the algebra structure in $\mathcal{H}$, i.e., none of these make $\mathcal{H}$ an $\mathcal{H}$-module algebra.

\section{$\diamond \quad \mathcal{H}$ acting on $\mathcal{F}$}

Let $X, Y$ be elements of $\mathcal{H}$, i.e., linear forms on $\mathcal{F}$, or even distributions on the would-be group $G$. Take $u \in \mathcal{F}$. Using the pairing $<,>$ between these dual Hopf-algebras, one can define actions of $\mathcal{H}$ on $\mathcal{F}$, that are dual to the ones on $\mathcal{H}$ we have just defined:

- the left action defined by

$$
<Y, X[u]>=<Y X, u>
$$

It comes from the right action of $\mathcal{H}$ on itself given by multiplication, $R[X] Y=Y X$. This is a left action of $\mathcal{H}$ on $\mathcal{F}$, since $(X Y)[u]=X[Y[u]$. It is also compatible with the algebra structure of $\mathcal{F}$ because $X[u v]=X_{1}[u] X_{2}[v]$, where $\Delta X=X_{1} \otimes X_{2}$ (implicit summation). Thus we also say that $\mathcal{F}$ is a left $\mathcal{H}$-module algebra. Notice that $\langle Y, X[u]\rangle=\langle Y X, u\rangle=$ $<Y \otimes X, \Delta u>=<Y \otimes X, u_{1} \otimes u_{2}>=<Y, u_{1}><X, u_{2}>$. Therefore, we have explicitly

$$
X[u]=u_{1}<X, u_{2}>
$$

- the left action defined by $\langle Y, X[u]\rangle=\langle S(X) Y, u>$, which is not compatible with the multiplication of $\mathcal{F}$ : more precisely, it involves a twist because $X[u v]=X_{2}[u] X_{1}[v]$. 
- the right action defined by

$$
<Y, X[u]>=<X Y, u>\text {. }
$$

It is obviously a right action on $\mathcal{F}$, since $(X Y)[u]=Y[X[u]]$.

- the right action defined by $<Y, X[u]\rangle=\langle Y S(X), u\rangle$.

Again, the first right action is compatible with the algebra structure of $\mathcal{F}$ whereas the second one involves a twist.

From now on, we shall choose consistently the left action of $\mathcal{H}$ on $\mathcal{F}$ defined by $\langle Y, X[u]\rangle=$ $\langle Y X, u\rangle$. Explicitly, we find for the left action of the generators $X_{ \pm}$and $K$ on $\mathcal{F}$

$$
\begin{array}{llllll}
X_{+}[a]=0 & X_{+}[b]=a & X_{-}[a]=b & X_{-}[b]=0 & K[a]=q a & K[b]=q^{2} b \\
X_{+}[c]=0 & X_{+}[d]=c & X_{-}[c]=d & X_{-}[d]=0 & K[c]=q c & K[d]=q^{2} d
\end{array}
$$

For every $X$ in $\mathcal{H}$, one can associate a linear operator $X[]$ from $\mathcal{F}$ to $\mathcal{F}$. This operator is sometimes called a "left invariant operator for the coproduct $\Delta$ " because $\Delta \circ X[]=(i d \otimes X[]) \circ \Delta$, where $\circ$ denotes the composition of maps and $i d$ is the identity map.

Indeed, $\left.\left.\Delta(X[u])=\Delta\left(u_{1}\right)<X, u_{2}\right\rangle=\left(u_{11} \otimes u_{12}\right)<X, u_{2}\right\rangle=(i d \otimes i d \otimes<X, \cdot>)\left(u_{11} \otimes u_{12} \otimes u_{2}\right)$. But $\left(u_{11} \otimes u_{12} \otimes u_{2}\right)=\left(u_{1} \otimes u_{21} \otimes u_{22}\right)$, because of the coassociativity of $\Delta$ (recall that $\left.(\Delta \otimes i d) \circ \Delta=(i d \otimes \Delta) \circ \Delta\right)$. So $\left.\Delta(X[u])=(i d \otimes i d \otimes<X, \cdot>)\left(u_{1} \otimes u_{21} \otimes u_{22}\right)=u_{1} \otimes u_{21}<X, u_{22}\right\rangle=(i d \otimes X[])\left(u_{1} \otimes u_{2}\right)=$ $(i d \otimes X[]) \Delta(u)$.

The space of left invariant operators on $\mathcal{F}$ - the dual of $\mathcal{H}$ - is isomorphic with $\mathcal{H}$ itself since $(X Y)[u]=X[Y[u]]$. Explicitly, the isomorphism $X \leadsto X[]$ and $X[] \leadsto X$ is given by $X[u]=$ $(i d \otimes\langle X, \cdot\rangle) \circ \Delta$ and $\langle X, u\rangle=\epsilon \circ X[u]$ where $\epsilon$ is the counit of $\mathcal{F}$. In a classical (i.e., group-like) situation, it would have been equivalent to evaluate $X[u]$ at the identity of the group to get $\langle X, u\rangle$.

On the contrary, the space of right invariant operators (for the coproduct $\Delta$ ) is anti-isomorphic with $\mathcal{H}$, since with a right action $(X Y)[u]=Y[X[u]]$.

\section{Remark about the classical case}

An easy way to remember the previous results is the following. First we introduce classical generators of $\operatorname{Lie}(S L(2))$ in the fundamental representation, i.e., the usual matrices $\underline{X}_{ \pm}, \underline{X}_{3}$ and define $\underline{K}=q \underline{X}_{3}:$

$$
\underline{X}_{+}=\left(\begin{array}{ll}
0 & 1 \\
0 & 0
\end{array}\right), \quad \underline{X}_{-}=\left(\begin{array}{cc}
0 & 0 \\
1 & 0
\end{array}\right), \quad \underline{X}_{3}=\left(\begin{array}{cc}
1 & 0 \\
0 & -1
\end{array}\right), \quad \underline{K}=q^{\underline{X}_{3}}=\left(\begin{array}{cc}
q & 0 \\
0 & q^{-1}
\end{array}\right) .
$$

Now we have a left action on $\mathcal{F}^{\text {class }}$, which for a generic $\underline{Y} \in \operatorname{Lie}(S L(2))$ reads]

$$
\underline{Y}^{L}\left[\left(\begin{array}{ll}
a & b \\
c & d
\end{array}\right)\right] \doteq\left(\begin{array}{ll}
a & b \\
c & d
\end{array}\right) \underline{Y}
$$

and a right one which is defined analogously. For the generators, this action reads explicitly

$$
\left(\begin{array}{ll}
a & b \\
c & d
\end{array}\right) \underline{X}_{+}=\left(\begin{array}{ll}
0 & a \\
0 & c
\end{array}\right), \quad\left(\begin{array}{ll}
a & b \\
c & d
\end{array}\right) \underline{X}_{-}=\left(\begin{array}{ll}
b & 0 \\
d & 0
\end{array}\right), \quad\left(\begin{array}{ll}
a & b \\
c & d
\end{array}\right) \underline{X}_{3}=\left(\begin{array}{ll}
a & -b \\
c & -d
\end{array}\right) .
$$

${ }^{5}$ Note that the following equations should be interpreted as equalities amongst entries of the matrices, i.e.,

$$
\underline{X}_{+}^{L}\left[\left(\begin{array}{ll}
a & b \\
c & d
\end{array}\right)\right] \equiv\left(\begin{array}{ll}
\underline{X}_{+}^{L}[a] & \underline{X}_{+}^{L}[b] \\
\underline{X}_{+}^{L}[c] & \underline{X}_{+}^{L}[d]
\end{array}\right) \doteq\left(\begin{array}{cc}
a & b \\
c & d
\end{array}\right) \underline{X}_{+},
$$

etc. This explains why this action is a left one, in spite of being given by a right multiplication; for example:

$$
\underline{X}_{+}^{L}\left[\underline{X}_{-}^{L}\left[\left(\begin{array}{cc}
a & b \\
c & d
\end{array}\right)\right]\right]=\underline{X}_{+}^{L}\left[\left(\begin{array}{cc}
b & 0 \\
d & 0
\end{array}\right)\right]=\left(\begin{array}{cc}
\underline{X}_{+}^{L}[b] & 0 \\
\underline{X}_{+}^{L}[d] & 0
\end{array}\right)=\left(\begin{array}{cc}
a & 0 \\
c & 0
\end{array}\right)=\left(\begin{array}{cc}
a & b \\
c & d
\end{array}\right) \underline{X}_{+} \underline{X}_{-}=\left(\underline{X}_{+} \underline{X}_{-}\right)^{L}\left[\left(\begin{array}{ll}
a & b \\
c & d
\end{array}\right)\right]
$$

This action is nothing more than the left action of the classical enveloping algebra of a Lie algebra on functions $U \in C(G)$ of the corresponding classical group $G$. Being dual to the right-product action $R[X] Y=Y X$, it is given infinitesimally for the Lie generators $x$ by $\left.\left.x[U]\right|_{g} \doteq U\right|_{g(\mathbf{1}+x)}-\left.U\right|_{g}$. 
Observe that $\underline{X}_{ \pm}$and $\underline{X}_{3}$ act by derivation on $\mathcal{F}^{\text {class }}$, and $\underline{K}$ as an automorphism. Indeed, the above left classical action (and the corresponding right one) of $\operatorname{Lie}(S L(2))$ on $\mathcal{F}^{\text {class }}$ may also be written in terms of operators (classical derivations)

$$
\begin{array}{llrl}
\underline{X}_{+}^{L} & =a \frac{\partial}{\partial b}+c \frac{\partial}{\partial d} & \underline{X}^{R} & =c \frac{\partial}{\partial a}+d \frac{\partial}{\partial b} \\
\underline{X}_{-}^{L} & =b \frac{\partial}{\partial a}+d \frac{\partial}{\partial c} & \underline{X}_{-}^{R} & =a \frac{\partial}{\partial c}+b \frac{\partial}{\partial d} \\
\underline{X}_{3}^{L} & =a \frac{\partial}{\partial a}-b \frac{\partial}{\partial b}+c \frac{\partial}{\partial c}-d \frac{\partial}{\partial d} & \underline{X}_{3}^{R} & =a \frac{\partial}{\partial a}+b \frac{\partial}{\partial b}-c \frac{\partial}{\partial c}-d \frac{\partial}{\partial d}
\end{array}
$$

It is easy to check that, for instance (and as it should!)

$$
\left[\underline{X}_{3}, \underline{X}_{+}\right]=+2 \underline{X}_{+}, \quad\left[\underline{X}_{3}^{L}, \underline{X}_{+}^{L}\right]=+2 \underline{X}_{+}^{L}, \quad\left[\underline{X}_{3}^{R}, \underline{X}_{+}^{R}\right]=-2 \underline{X}_{+}^{R} .
$$

We recover the fact that left fundamental vector fields build up, in the classical case, the Lie algebra itself.

As we see, it turns out that the formulae (16) expressing the action of $\mathcal{H}$ on the quantum generators $a, b, c, d$ are the same as the classical ones. However, the quantum action cannot be extended by derivations, when the same quantities act on arbitrary elements of $\mathcal{F}$ : the action of invariant linear operators is then carried out by twisted derivations (derivations twisted by automorphisms). This can be easily seen remembering that one should use the coproduct of $\mathcal{H}$ to act on products of $\mathcal{F}$.

\section{$\diamond \quad \mathcal{H}$ acting on $\mathcal{M}$}

Using the fact that $\mathcal{F}$ coacts on $\mathcal{M}$ (an algebra of matrices) in two possible ways, and that elements of $\mathcal{H}$ can be interpreted as distributions on $\mathcal{F}$, we obtain two actions of $\mathcal{H}$ on the quantum space $\mathcal{M}$. We shall describe them for arbitrary elements and give explicit results for the generators.

Let $z \in \mathcal{M}$. We know that $\mathcal{F}$ coacts on $\mathcal{M}$ from the left and from the right. For the left coaction, we have $\Delta_{L} z=u_{\alpha} \otimes z_{\alpha}$ with $u_{\alpha} \in \mathcal{F}$ and $z_{\alpha} \in \mathcal{M}$ (implied summation). For instance $\Delta_{L} x=a \otimes x+b \otimes y$. Let $X$ be an element of $\mathcal{H}$. Using the pairing between $\mathcal{H}$ and $\mathcal{F}$, we set

$$
X^{R}[z] \doteq(<X, \cdot>\otimes i d) \Delta_{L} z=<X, u_{\alpha}>z_{\alpha},
$$

which makes it into a right action of $\mathcal{H}$ on $\mathcal{M}$. Moreover, it makes $\mathcal{M}$ a right- $\mathcal{H}$-module algebra. For instance, $X_{+}^{R}[x]=<X_{+}, a>x+<X_{+}, b>y=0 x+1 y=y$.

$\mathcal{F}$ also coacts on $\mathcal{M}$ from the right, so that $\Delta_{R} z=z_{\alpha} \otimes v_{\alpha}$ with $z_{\alpha} \in \mathcal{M}$ and $v_{\alpha} \in \mathcal{F}$ (implied summation). For instance $\Delta_{R} x=x \otimes a+y \otimes c$. Taking $X \in \mathcal{H}$, and using the $\mathcal{H}-\mathcal{F}$ pairing we can define the left action

$$
X^{L}[z] \doteq(i d \otimes<X, \cdot>) \Delta_{R} z=<X, v_{\alpha}>z_{\alpha} .
$$

With this $L$-action we can check that $\mathcal{M}$ a left- $\mathcal{H}$-module algebra. In particular, $X_{+}^{L}[x]=x<$ $X_{+}, a>+y<X_{+}, c>=0 x+0 y=0$.

We stress again that we have denoted with $X^{L}$ the operators on $\mathcal{M}$ corresponding to the leftaction of an element $X \in \mathcal{H}$ on $\mathcal{M}$, but this action is indeed dual to the right-coaction of $\mathcal{F}$ on $\mathcal{M}$. The same happens for the right-action. One should therefore use the notation involving upper indices $L$ or $R$ with some care.

To obtain the action of an arbitrary element $X \in \mathcal{H}$ on a product $z w$ of elements of $\mathcal{M}$, one may use the definition (above) or the following property (notation should be clear):

$$
X^{L}[z w]=m\left[(\Delta X)^{L}[z \otimes w]\right] .
$$

It is therefore enough to know the action of the generators $X_{ \pm}, K$ on $\mathcal{M}$ (complete $27 \times 27$ tables, with other conventions, can be found in [2]). One finds: 


\begin{tabular}{l||ccc} 
Right & $K^{R}$ & $X_{+}^{R}$ & $X_{-}^{R}$ \\
\hline \hline $\mathbb{1}$ & $\mathbb{1}$ & 0 & 0 \\
$x^{2} y$ & $q x^{2} y$ & $-x y^{2}$ & $\mathbb{1}$ \\
$x y^{2}$ & $q^{2} x y^{2}$ & $\mathbb{1}$ & $-x^{2} y$ \\
\hline$x$ & $q x$ & $y$ & 0 \\
$y$ & $q^{2} y$ & 0 & $x$ \\
$x^{2} y^{2}$ & $x^{2} y^{2}$ & $-x$ & $-y$ \\
\hline$x^{2}$ & $q^{2} x^{2}$ & $-x y$ & 0 \\
$x y$ & $x y$ & $y^{2}$ & $x^{2}$ \\
$y^{2}$ & $q y^{2}$ & 0 & $-x y$
\end{tabular}

\begin{tabular}{l||ccc} 
Left & $K^{L}$ & $X_{+}^{L}$ & $X_{-}^{L}$ \\
\hline \hline $\mathbb{1}$ & $\mathbb{1}$ & 0 & 0 \\
$x^{2} y$ & $q x^{2} y$ & $q^{2} \mathbb{1}$ & $-x y^{2}$ \\
$x y^{2}$ & $q^{2} x y^{2}$ & $-x^{2} y$ & $q^{2} \mathbb{1}$ \\
\hline$x$ & $q x$ & 0 & $y$ \\
$y$ & $q^{2} y$ & $x$ & 0 \\
$x^{2} y^{2}$ & $x^{2} y^{2}$ & $-q y$ & $-q x$ \\
\hline$x^{2}$ & $q^{2} x^{2}$ & 0 & $-q^{2} x y$ \\
$x y$ & $x y$ & $q x^{2}$ & $q y^{2}$ \\
$y^{2}$ & $q y^{2}$ & $-q^{2} x y$ & 0
\end{tabular}

Table 1: Right and left actions of $\mathcal{H}$ on $\mathcal{M}$.

Notice that, up to multiplicative factors,
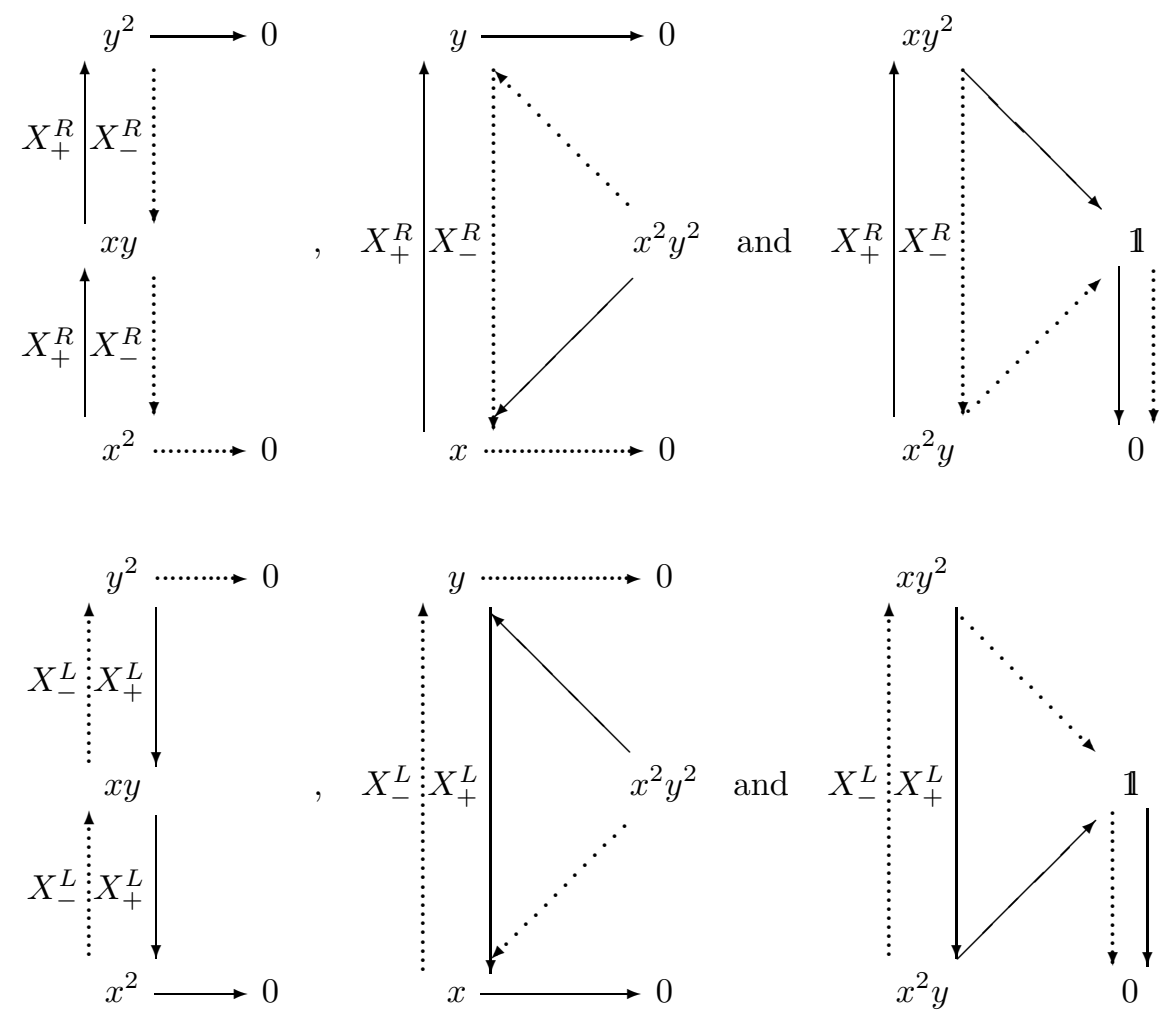

We see clearly on these diagrams that (up to numerical factors) the right action is obtained from the left one by interchanging arrows for $X_{+}$and $X_{-}$.

\section{Differential operators on $\mathcal{M}$ associated with the $\mathcal{H}$ action}

Here, we are only concerned with those differential operators on $\mathcal{M}$ associated with the quantum group action of $\mathcal{H}$. A more complete analysis can be found in Appendix $\mathrm{F}$. They are obtained by considering products and powers of $X_{ \pm}^{L, R}, K^{L, R}$ acting from the left or from the right as before. From now on we will stick with the left action.

We also consider elements of $\mathcal{M}$ acting by multiplication as differential operators of order zero: let $z \in \mathcal{M}$, then, for example, $x[z] \doteq x z$. Let $X \in \mathcal{H}$; the reader should distinguish between $X x$-which is a differential operator on $\mathcal{M}(X x[z] \doteq X[x[z]]=X[x z])$ - from $X[x]$ which is an element of $\mathcal{M}$. It makes therefore perfect sense to study the commutation relations, say, between 
$X$ and $x$. We shall use such relations later. Here, we establish the commutation relations between $x, y$ - taken as differential operators of degree 0 - and $X_{ \pm}, K$.

Let $z \in \mathcal{M}$. Consider, for instance,

$$
X_{+}^{L}[x z]=X_{+}^{L}[x] \mathbb{1}[z]+K^{L}[x] X_{+}^{L}[z]=0+q x X_{+}^{L}[z] .
$$

Therefore $X_{+}^{L} x=q x X_{+}^{L}$. Similarly, we obtain:

$$
\begin{aligned}
X_{+}^{L} x & =q x X_{+}^{L} \\
X_{-}^{L} x & =y K^{L^{-1}}+x X_{-}^{L} \\
K^{L} x & =q x K^{L} \\
X_{+}^{L} y & =x+q^{2} y X_{+}^{L} \\
X_{-}^{L} y & =y X_{-}^{L} \\
K^{L} y & =q^{2} y K^{L}
\end{aligned}
$$

Any element of $\mathcal{H}$ can also be written explicitly in terms of differential operators on $\mathcal{M}$; see Appendix F.

It may be useful to notice that it is possible to introduce "conformal weights" so that previous formulae are homogeneous with the following weight assignments: $\left(X_{+}^{L}, K^{L}, X_{-}^{L}\right) \mapsto(1,0,-1)$, and $(x, y) \mapsto(1 / 2,-1 / 2)$.

\subsection{The structure of the non-semisimple algebra $\mathcal{H}$}

The algebra $\mathcal{H}$ can be explicitly defined - and many of its properties understood - without using anything more sophisticated than basic multiplication or tensor products of matrices as well as elementary calculus involving anti-commuting numbers (Grassmann numbers). This construction, inspired from [3], was explicitly performed in [4] for $N=3$ and used to analyse the representation theory of $\mathcal{H}$. The analysis for other values of $N$ can be found in [9]. We just recall here the results and establish the relations with the previous notations.

The finite-dimensional quantum group $\mathcal{H}$ can be defined as the algebra $\mathcal{H} \doteq M_{3} \oplus\left(M_{2 \mid 1}\left(\Lambda^{2}\right)\right)_{0}$, where $M_{3}$ is the set of $3 \times 3$ complex matrices and $\left(M_{2 \mid 1}\left(\Lambda^{2}\right)\right)_{0}$ is the Grassmann envelope of the associative $\mathbb{Z}_{2}$-graded algebra $M_{2 \mid 1}\left(\mathbb{C}^{2}\right)$, i.e., the even part of its graded tensor product with a Grassmann algebra $\Lambda^{2}$ of two generators.

To see this explicitly, let $\Lambda^{2}$ be the Grassmann algebra over $\mathbb{C}$ with two generators, i.e., the linear span of $\left\{1, \theta_{1}, \theta_{2}, \theta_{1} \theta_{2}\right\}$ with arbitrary complex coefficients, where the relations $\theta_{1}^{2}=\theta_{2}^{2}=0$ and $\theta_{1} \theta_{2}=-\theta_{2} \theta_{1}$ hold. This algebra has an even part, generated by 1 and $\theta_{1} \theta_{2}$, and an odd part, generated by $\theta_{1}$ and $\theta_{2}$. We call $M_{3}$ the algebra of $3 \times 3$ matrices over the complex numbers and $M_{2 \mid 1}$ another copy of this algebra that we grade as follows: a matrix $V \in M_{2 \mid 1}$ is called even if it is of the type

$$
V=\left(\begin{array}{ccc}
V_{11} & V_{12} & 0 \\
V_{21} & V_{22} & 0 \\
0 & 0 & V_{33}
\end{array}\right),
$$

and odd if it is of the type

$$
V=\left(\begin{array}{ccc}
0 & 0 & V_{13} \\
0 & 0 & V_{23} \\
V_{31} & V_{32} & 0
\end{array}\right) .
$$

We call $\left(M_{2 \mid 1}\left(\Lambda^{2}\right)\right)_{0}$ the Grassmann envelope of $M_{2 \mid 1}$ which is defined as the even part of the tensor product of $M_{2 \mid 1}$ and $\Lambda^{2}$, i.e., the space of $3 \times 3$ matrices $V$ with entries $V_{11}, V_{12}, V_{21}, V_{22}, V_{33}$ that

are even Grassmann elements (of the kind $\alpha+\beta \theta_{1} \theta_{2}$ ) and entries $V_{13}, V_{23}, V_{31}, V_{32}$ that are odd Grassmann elements (of the kind $\gamma \theta_{1}+\delta \theta_{2}$ ). We define $\mathcal{H}$ as

$$
\mathcal{H} \doteq M_{3} \oplus\left(M_{2 \mid 1}\left(\Lambda^{2}\right)\right)_{0} .
$$


Explicitly,

$$
\mathcal{H}=\left(\begin{array}{ccc}
* & * & * \\
* & * & * \\
* & * & *
\end{array}\right) \oplus\left(\begin{array}{ccc}
\alpha_{11}+\beta_{11} \theta_{1} \theta_{2} & \alpha_{12}+\beta_{12} \theta_{1} \theta_{2} & \gamma_{13} \theta_{1}+\delta_{13} \theta_{2} \\
\alpha_{21}+\beta_{21} \theta_{1} \theta_{2} & \alpha_{22}+\beta_{22} \theta_{1} \theta_{2} & \gamma_{23} \theta_{1}+\delta_{23} \theta_{2} \\
\gamma_{31} \theta_{1}+\delta_{31} \theta_{2} & \gamma_{32} \theta_{1}+\delta_{32} \theta_{2} & \alpha_{33}+\beta_{33} \theta_{1} \theta_{2}
\end{array}\right)
$$

All entries besides the $\theta$ 's are complex numbers (the above $\oplus$ sign is a direct sum sign: these matrices are $6 \times 6$ matrices written as a direct sum of two blocks of size $3 \times 3$ ).

It is obvious that this is an associative algebra, with usual matrix multiplication, of dimension 27 (just count the number of arbitrary parameters). $\mathcal{H}$ is not semisimple, because of the appearance of Grassmann numbers in the entries of the matrices. Its semisimple part $\overline{\mathcal{H}}$, given by the direct sum of its block-diagonal $\theta$-independent parts is equal to the $9+4+1=14$-dimensional algebra $\overline{\mathcal{H}}=M_{3}(\mathbb{C}) \oplus M_{2}(\mathbb{C}) \oplus \mathbb{C}$. The radical (more precisely the Jacobson radical) $J$ of $\mathcal{H}$ is the left-over piece that contains all the Grassmann entries, and only the Grassmann entries, so $\overline{\mathcal{H}}=\mathcal{H} / J$. The radical has therefore dimension 13.

In order to write generators for $\mathcal{H}$, we need to consider $6 \times 6$ matrices that have a $(3 \times 3) \oplus$ $((2 \mid 1) \times(2 \mid 1))$ block diagonal structure. We introduce elementary matrices $E_{i j}$ for the $M_{3}(\mathbb{C})$ part and elementary matrices $F_{i j}$ for the $M_{2 \mid 1}(\mathbb{C})$ part. The associative algebra $\mathcal{H}$ defined previously can be generated by the following three matrices:

$$
\begin{aligned}
X_{+} & =E_{12}+E_{23}+\left(1-\theta_{1} \theta_{2} / 2\right) F_{12}+\theta_{1}\left(F_{23}+F_{31}\right) \\
X_{-} & =-E_{21}-E_{32}+\left(1-\theta_{1} \theta_{2} / 2\right) F_{21}+\theta_{2}\left(F_{13}-F_{32}\right) \\
K & =q^{2} E_{11}+E_{22}+q^{-2} E_{33}+q F_{11}+q^{-1} F_{22}+F_{33}
\end{aligned}
$$

Explicitly, one gets

$$
\begin{aligned}
& X_{+} \doteq\left(\begin{array}{ccc}
\left(\begin{array}{lll}
0 & 1 & 0 \\
0 & 0 & 1 \\
0 & 0 & 0
\end{array}\right) & () & \\
& (~) & \left(\begin{array}{ccc}
0 & 1-\frac{\theta_{1} \theta_{2}}{2} & 0 \\
0 & 0 & \theta_{1} \\
\theta_{1} & 0 & 0
\end{array}\right)
\end{array}\right)
\end{aligned}
$$

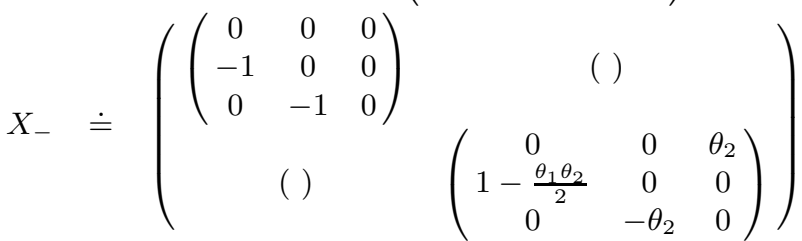

$$
\begin{aligned}
& K \doteq\left(\begin{array}{ccc}
\left(\begin{array}{ccc}
q^{2} & 0 & 0 \\
0 & 1 & 0 \\
0 & 0 & q^{-2}
\end{array}\right) & (\text { ) } \\
& (\text { ) } & \left.\left(\begin{array}{ccc}
q & 0 & 0 \\
0 & q^{-1} & 0 \\
0 & 0 & 1
\end{array}\right)\right)
\end{array}\right.
\end{aligned}
$$

Performing explicit matrix multiplications or using the relations $E_{i j} E_{j k}=E_{i k}, F_{i j} F_{j k}=F_{i k}$ and $E_{i j} F_{j k}=F_{i j} E_{j k}=0$, it is easy to see that the defining relations of $\mathcal{H}$ are indeed satisfied.

As mentioned in Section 4.2, the center of $\mathcal{H}$ is generated by a Casimir operator $C$. Its explicit expression reads

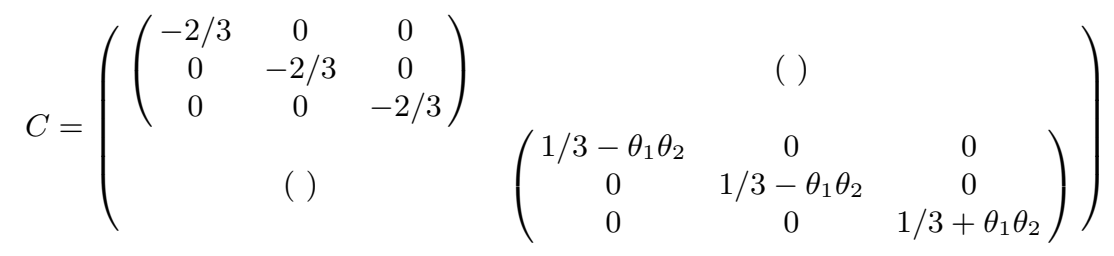


The problem of studying representation theory for $\mathcal{H}$ is solved by considering separately all the columns defining $\mathcal{H}=M_{3} \oplus\left(M_{2 \mid 1}\left(\Lambda^{2}\right)\right)_{0}$ as a matrix algebra over a ring. We just "read" the following three indecomposable representations from the explicit definition of $\mathcal{H}$. First of all we have $\sqrt{6}$ a three-dimensional irreducible representation $3_{i r r} \doteq\left(c_{1}, c_{2}, c_{3}\right)$ (where $c_{i}$ are complex numbers) coming from the $M_{3}$ block. Notice that the three columns of the $M_{3}$ factor give equivalent irreducible representations. Next we have two reducible indecomposable representations (also called "PIM's" for "Projective Indecomposable Modules") coming from the columns of $\left(M_{2 \mid 1}\left(\Lambda^{2}\right)\right)_{0}$. Notice that the first two columns give equivalent representations - that we call $P_{e} \equiv 6_{\text {eve }}$ - and the last one gives the representation $P_{o} \equiv 6_{\text {odd }}$. Each of these two PIM's is of dimension 6 . The PIM's are also called "principal modules".

$P_{o}$ and $P_{e}$, although indecomposable, are not irreducible: submodules (sub-representations) are immediately found by requiring stability of the representation spaces under the left multiplication by elements of $\mathcal{H}$. The lattice of submodules of $\mathcal{H}$ is given and discussed in 顿. We recall these results in Appendix D. Here we just want to note (because it will be useful shortly) that $6_{o}$ and $6_{e}$ both contain indecomposable (but not irreducible) sub-representations of dimension 3 . Indeed, take $\lambda_{1}, \lambda_{2} \in \mathbb{C}$, set $\lambda \doteq \frac{\lambda_{1}}{\lambda_{2}} \in C P^{1}$, and define $\theta_{\lambda}=\lambda_{1} \theta_{1}+\lambda_{2} \theta_{2}$. We obtain, for each $\lambda$, a representation $3_{\text {odd }}$ spanned by $\left(\gamma \theta_{\lambda}, \gamma^{\prime} \theta_{\lambda}, \alpha \theta_{1} \theta_{2}\right)$ and a representation $3_{\text {eve }}$ spanned by $\left(\beta \theta_{1} \theta_{2}, \beta^{\prime} \theta_{1} \theta_{2}, \delta \theta_{\lambda}\right)$. Here the coefficients $\beta, \beta^{\prime}, \gamma, \gamma^{\prime}, \alpha, \delta$ are arbitrary complex numbers.

\subsection{Decomposition of $\mathcal{M}=M_{3}(\mathbb{C})$ into representations of $\mathcal{H}$}

Since there is an action of $\mathcal{H}$ on $\mathcal{M}$, it is a priori clear that $\mathcal{M}$, as a vector space, can be analysed in terms of representations of $\mathcal{H}$. It turns out, as it is easy to see from (19) or (20), that

$$
\mathcal{M} \sim 3_{\text {irr }} \oplus 3_{\text {eve }} \oplus 3_{\text {odd }} .
$$

More precisely,

- $3_{\text {irr }}$ is spanned by $x^{2}, x y, y^{2}$,

- $3_{\text {eve }}$ is spanned by $x, y, x^{2} y^{2}$,

- $3_{\text {odd }}$ is spanned by $\mathbb{1}, x^{2} y, x y^{2}$.

If we attribute a $\mathbb{Z}_{3}$-grading 1 to $x$ and $y$, these three spaces carry gradings respectively equal to $2,1,0$. This same analysis can be done in a more general $N$ case, the interested reader can find it in [11.

The algebra $\mathcal{M}$, as a vector space, carries therefore a 9-dimensional reducible representation of $\mathcal{H}$ and splits into the direct sum of an irreducible representation of dimension 3 and two inequivalent representations of dimension 3 that are reducible but not fully reducible. As described previously, these last two representations $3_{\text {eve }}$ and $3_{\text {odd }}$ appear as subrepresentations of two projective indecomposable modules of dimension 6 . It is easy to see (cf. diagrams below) that $3_{\text {eve }}=2 \oplus 1$ where 2 denotes a two-dimensional invariant subspace (but 1 is not) whereas $3_{\text {odd }}=1 \oplus 2$ where 1 denotes a one-dimensional invariant subspace (but 2 is not).

One possible identification between $\mathcal{H}$ acting from the left on $\mathcal{M}$ and $3_{\text {irr }} \oplus 3_{\text {eve }} \oplus 3_{\text {odd }}$ as elements of the left regular representation of $\mathcal{H}$, is as follows:

$$
\begin{array}{rlrlrl}
x^{2} & \sim E_{12} & x & \sim \theta_{1} \theta_{2} F_{11} & x^{2} y & \sim\left(\theta_{1}-\theta_{2}\right) F_{13} \\
x y & \sim E_{22} & y & \sim \theta_{1} \theta_{2} F_{21} & x y^{2} \sim\left(\theta_{1}-\theta_{2}\right) F_{23} \\
y^{2} & \sim E_{32} & x^{2} y^{2} & \sim\left(\theta_{1}-\theta_{2}\right) F_{31} & & \mathbb{1} \sim \theta_{1} \theta_{2} F_{33}
\end{array}
$$

In order to establish this identification, it is enough to compare the values of $X^{L}[z]$, for the generators $X$ of $\mathcal{H}$ and $z \in \mathcal{M}$, with those obtained by left matrix multiplication using the above realisation of $\mathcal{H}$ as $M_{3} \oplus\left(M_{2 \mid 1}\left(\Lambda^{2}\right)\right)_{0}$ (of course, we could use right matrix multiplication and compare with the values of $X^{R}[z]$ given before). For instance, in the case of the generator $X_{+}$we obtain

$$
\begin{array}{ccc}
X_{+} E_{12}=0 & X_{+} \theta_{1} \theta_{2} F_{11}=0 & X_{+}\left(\theta_{1}-\theta_{2}\right) F_{13}=-\theta_{1} \theta_{2} F_{33} \\
X_{+} E_{22}=E_{12} & X_{+} \theta_{1} \theta_{2} F_{21}=\theta_{1} \theta_{2} F_{11} & X_{+}\left(\theta_{1}-\theta_{2}\right) F_{23}=\left(\theta_{1}-\theta_{2}\right) F_{13} \\
X_{+} E_{32}=E_{22} & X_{+}\left(\theta_{1}-\theta_{2}\right) F_{31}=-\theta_{1} \theta_{2} F_{21} & X_{+} \theta_{1} \theta_{2} F_{33}=0
\end{array}
$$

${ }^{6}$ The following should be read as "column vectors". 
to be compared with

$$
\begin{array}{cccrl}
X_{+}^{L}\left[x^{2}\right]=0 & X_{+}^{L}[x] & =0 & X_{+}^{L}[\mathbb{1}]=0 \\
X_{+}^{L}[x y]=q x^{2} & X_{+}^{L}[y] & =x & X_{+}^{L}\left[x^{2} y\right]=q^{2} \mathbb{1} \\
X_{+}^{L}\left[y^{2}\right]=-q^{2} x y & X_{+}^{L}\left[x^{2} y^{2}\right] & =-q y & X_{+}^{L}\left[x y^{2}\right]=-x^{2} y
\end{array}
$$

One obtains in this way two identical $3 \times 9$ tables.

This can also be seen by comparing the following diagrams with those obtained previously:
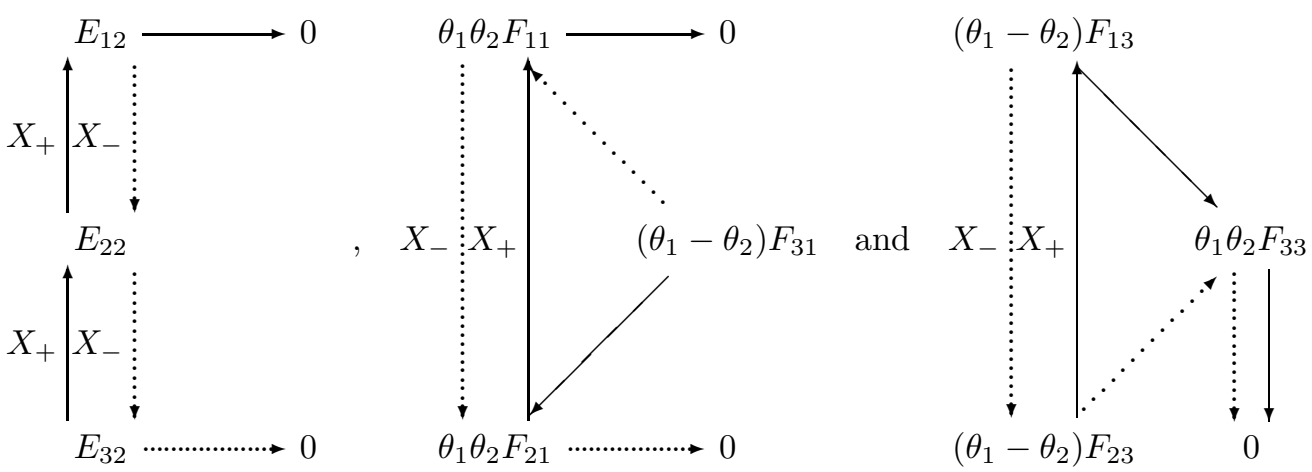


\section{$5 \quad$ Reality structures}

We now want to obtain a reality structure (a star) and a scalar product on the space of matrices $\mathcal{M}$, with covariance properties under the quantum group transformations.

\subsection{Real forms and stars on quantum groups}

A $*$-Hopf algebra $\mathcal{F}^{\prime}$ is an associative algebra that satisfies the following properties (for all elements $a, b$ in $\left.\mathcal{F}^{\prime}\right)$ :

- $\mathcal{F}^{\prime}$ is a Hopf algebra (a quantum group), with coproduct $\Delta$, antipode $S$ and counit $\epsilon$.

- $\mathcal{F}^{\prime}$ is an involutive algebra, i.e., it has an involution $*$ (a 'star' operation). This operation is involutive $(* * a=a)$, antilinear $(*(\lambda a)=\bar{\lambda} * a$, where $\lambda$ is a complex number), and anti-multiplicative $(*(a b)=(* b)(* a))$.

- The involution is compatible with the coproduct, in the following sense: if $\Delta a=a_{1} \otimes a_{2}$, then $\Delta * a=* a_{1} \otimes * a_{2}$.

- The involution is also compatible with the counit: $\epsilon(* a)=\overline{\epsilon(a)}$.

- The following relation with the antipode holds: $S * S * a=a$.

Actually, the last relation is a consequence of the others. It can also be written

$$
S *=* S^{-1} .
$$

It may happen that $S^{2}=i d$, in which case $S *=* S$, but it is not generally so (usually the square of the antipode is only a conjugacy).

If one wishes, using the $*$ on $\mathcal{F}^{\prime}$, one can define a star operation on the tensor product $\mathcal{F}^{\prime} \otimes \mathcal{F}^{\prime}$, by $*(a \otimes b)=* a \otimes * b$. The third condition reads then:

$$
\Delta *=* \Delta
$$

so one can say $\Delta$ is a $*$-homomorphism between $\mathcal{F}^{\prime}$ and $\mathcal{F}^{\prime} \otimes \mathcal{F}^{\prime}$ (each with its respective star). It can also be said that $\epsilon$ is a $*$-homomorphism between $\mathcal{F}^{\prime}$ and $\mathbb{C}$ with the star given by complex conjugation.

A star operation as above, making the Hopf-algebra a $*$-Hopf algebra, is also called a real form for the given algebra. Very often, it is convenient to write $a^{*} \doteq * a$. A real element is, by definition, an element such that $a^{*}=a$. Notice that a product of real elements $a$ and $b$ is not real in general $\left((a b)^{*}=b^{*} a^{*}=b a\right)$, but the anticommutator of two real elements, i.e., the Jordan product, is real $\left((a b+b a)^{*}=b^{*} a^{*}+a^{*} b^{*}=a b+b a\right)$.

\subsubsection{Twisted stars}

It may happen that one finds an involution $*$ on a Hopf algebra for which the third axiom fails in a special way, namely, the case where $\Delta a=a_{1} \otimes a_{2}$ but $\Delta * a=* a_{2} \otimes * a_{1}$. In this case $S *=* S$. Such an involution is called a twisted star operation. Remember that, whenever one has a coproduct $\Delta$ on an algebra, it is possible to construct another coproduct $\Delta^{o p}$ by composing the first one with the tensorial flip: if $\Delta a=a_{1} \otimes a_{2}$, then $\Delta^{o p} a \doteq a_{2} \otimes a_{1}$. If one defines a star operation on the tensor product (as before) by $*(a \otimes b) \doteq * a \otimes * b$, the property defining a twisted star reads

$$
\Delta *=* \Delta^{o p} \text {. }
$$

One should be cautious: some authors introduce a different star operation on the tensor product, namely $*^{\prime}(a \otimes b) \doteq * b \otimes * a$. In that case, a twisted star operation obeys $\Delta *=*^{\prime} \Delta$ !

*-Hopf algebras seem to be "better" than Hopf algebras endowed with twisted stars (their representation theory is more interesting). In any case we shall only be interested in genuine "true" *-Hopf algebras and the only reason why we mention these twisted stars here is that we want to rule out some of the possibilities that we shall meet later. 


\subsection{General results concerning real forms for the classical group $S L(2, \mathbb{C})$ and the quantum group $\operatorname{Fun}\left(S L_{q}(2, \mathbb{C})\right)$}

The following results are known and can be found easily in the literature. Classically, one can define several real forms for $S L(2, \mathbb{C})$, by selecting the elements which are unitary with respect to a given scalar product $G$, which defines a conjugacy on the group. This last one, in turn, may be thought as coming from a real form on the universal enveloping algebra of the Lie algebra of the group. If $u=\left(\begin{array}{ll}\alpha & \beta \\ \gamma & \delta\end{array}\right) \in S L(2, \mathbb{C})$, one may impose $\overline{u^{t}} G u=G$. There are three cases:

1. Here $G=\left(\begin{array}{ll}1 & 0 \\ 0 & 1\end{array}\right)$. The corresponding real form is called $S U(2)$. The matrix elements obey $\alpha^{*}=\delta, \beta^{*}=-\gamma, \gamma^{*}=-\beta$ and $\delta^{*}=\alpha$.

2. Here $G=\left(\begin{array}{cc}1 & 0 \\ 0 & -1\end{array}\right)$. The corresponding real form is called $S U(1,1)$. The matrix elements obey $\alpha^{*}=\delta, \beta^{*}=\gamma, \gamma^{*}=\beta$ and $\delta^{*}=\alpha$.

3. Here $G=\left(\begin{array}{ll}0 & 1 \\ 1 & 0\end{array}\right)$. The corresponding real form is called $S L(2, \mathbb{R})$. The matrix elements obey $\alpha^{*}=\alpha, \beta^{*}=\beta, \gamma^{*}=\gamma$ and $\delta^{*}=\delta$.

In the classical case, $S U(2)$ and $S L(2, \mathbb{R})$ have the same finite-dimensional representations (they do not have the same infinite-dimensional ones). For this reason there is a one to one correspondence between the Hopf algebras of these two groups (indeed, the Hopf algebra of a group can be considered as the space of polynomials on matrix elements of finite-dimensional representations). The star operation allows one to distinguish between the two groups. Moreover, in the classical case, the two groups $S U(1,1)$ and $S L(2, \mathbb{R})$ are isomorphic. One could also consider the complex group $S L(2, \mathbb{C})$ itself as a real group (the spin covering of the Lorentz group) but the dimensionality is twice bigger and we are not going to follow this route in the quantum case - at least not in the present paper.

In the quantum case, one has also three possibilities for the star operations on $\operatorname{Fun}\left(S L_{q}(2, \mathbb{C})\right)$ (up to Hopf automorphisms):

1. The real form $\operatorname{Fun}\left(S U_{q}(2)\right)$. The matrix elements obey $a^{*}=d, b^{*}=-q c, c^{*}=-q^{-1} b$ and $d^{*}=a$. Moreover, $q$ should be real.

2. The real form $\operatorname{Fun}\left(S U_{q}(1,1)\right)$. The matrix elements obey $a^{*}=d, b^{*}=q c, c^{*}=q^{-1} b$ and $d^{*}=a$. Moreover, $q$ should be real.

3. The real form $\operatorname{Fun}\left(S L_{q}(2, \mathbb{R})\right)$. The matrix elements obey $a^{*}=a, b^{*}=b, c^{*}=b$ and $d^{*}=d$. Here $q$ can be complex but it should be a phase.

In the quantum case, it is already clear from these results that taking $q$ a root of unity is incompatible with the $S U_{q}$ real forms, and that the only possibility if we assume $q^{N}=1$ is to choose the star corresponding to $\operatorname{Fun}\left(S L_{q}(2, \mathbb{R})\right)$. Notice also that, in the quantum case, the two real forms $\operatorname{Fun}\left(S U_{q}(1,1)\right)$ and $\operatorname{Fun}\left(S L_{q}(2, \mathbb{R})\right)$ are different (already the range of values where $q$ can be chosen do not coincide).

\subsection{Real forms on $\mathcal{F}$}

Having only one real form compatible with a complex $q$ on $F u n\left(S L_{q}(2, \mathbb{C})\right)$ already tells us that there is at most one real form on its quotient $\mathcal{F}$. We only have to check that the star operation preserves the ideal and coideal defined by $a^{3}=d^{3}=\mathbb{1}, b^{3}=c^{3}=0$. This is trivial because $a^{*}=a$, $b^{*}=b, c^{*}=c$ and $d^{*}=d$. Hence, this star operation can be restricted to $\mathcal{F}$.

This real form can be considered as a reduced quantum group associated with the real form $\operatorname{Fun}\left(S L_{q}(2, \mathbb{R})\right)$ of $\operatorname{Fun}\left(S L_{q}(2, \mathbb{C})\right)$.

\subsection{Real structures and star operations on $\mathcal{M}, \mathcal{H}$}

Now we would like to introduce an involution (a star operation) on the comodule algebra $\mathcal{M}$. This involution should be compatible with the coaction of $\mathcal{F}$. That is, we are asking for covariance of 
the star under the (right,left) $\mathcal{F}$-coaction,

$$
\left(\Delta_{R, L} z\right)^{*}=\Delta_{R, L}\left(z^{*}\right), \quad \text { for any } z \in \mathcal{M} .
$$

Here we have also used the notation $*$ for the star on the tensor products, which is defined as $(A \otimes B)^{*}=A^{*} \otimes B^{*}$. Using, for instance, the left coaction $\left(\begin{array}{l}x^{\prime} \\ y^{\prime}\end{array}\right)=\left(\begin{array}{ll}a & b \\ c & d\end{array}\right) \otimes\left(\begin{array}{l}x \\ y\end{array}\right)$ in (22), we see immediately that $\operatorname{Fun}\left(S U_{q}(2)\right)$ corresponds to the choice $x^{*}=y, y^{*}=-q^{-1} x$. Writing $x y=q y x$, one also recovers the fact that, in this case, $q$ should be real. In the same way, we find that the real form $\operatorname{Fun}\left(S U_{q}(1,1)\right)$ corresponds to the choice $x^{*}=y, y^{*}=q^{-1} x$. Finally, the real form $\operatorname{Fun}\left(S L_{q}(2, \mathbb{R})\right)$ corresponds to $x^{*}=x$ and $y^{*}=y$.

As $q^{3}=1$, we stick to the $\operatorname{Fun}\left(S L_{q}(2, \mathbb{R})\right)$ case since it is the only one compatible with a complex $q$. We therefore define the star on $\mathcal{M}=M_{3}(\mathbb{C})$ by

$$
x^{*}=x, \quad y^{*}=y .
$$

We now want to find a compatible $*$ on the algebra $\mathcal{H}$. Of course, one could proceed by using duality with $\mathcal{F}$, but it is both handy and instructive to proceed otherwise.

We let $\mathcal{M}$ act on itself by multiplication and we want to promote this star operation to an involution defined for all operators acting on $\mathcal{M}$. In particular, on the operators determined by the action of $\mathcal{H}$, given in Section 4.4. To this end, we apply the $*$ operation to the commutation relations between $x, y$ and the differential operators $X^{L}$ (or $X^{R}$ ), and read off the results. For instance, $X_{+}^{L} y=x+q^{2} y X_{+}^{L}$ implies $y\left(X_{+}^{L}\right)^{*}=x+\overline{q^{2}}\left(X_{+}^{L}\right)^{*} y$ and this suggests $\left(X_{+}^{L}\right)^{*}=-q^{2}\left(X_{+}^{L}\right)$. In this way we obtain:

$$
\begin{aligned}
& \left(X_{+}^{L}\right)^{*}=-q^{2} X_{+}^{L} \\
& \left(X_{-}^{L}\right)^{*}=-q X_{-}^{L} \\
& \left(K^{L}\right)^{*}=K^{L}
\end{aligned}
$$

and

$$
\begin{aligned}
& \left(X_{+}^{R}\right)^{*}=-q X_{+}^{R} \\
& \left(X_{-}^{R}\right)^{*}=-q^{2} X_{-}^{R} \\
& \left(K^{R}\right)^{*}=K^{R}
\end{aligned}
$$

Notice the change of $q$ into $q^{-1}$ when going from left to right. As the space of operators coming from a left action is isomorphic with $\mathcal{H}$ itself, the star operation in $\mathcal{H}$ is the same as the one obtained for the operators $X_{ \pm}^{L}, K^{L}$, namely

$$
X_{+}^{*}=-q^{2} X_{+}, \quad X_{-}^{*}=-q X_{-}, \quad K^{*}=K .
$$

Now, of course, one has to check that this involution satisfies all the axioms given at the beginning of this section. This is rather straightforward. For illustration, let us compute $\Delta\left(X_{-}^{*}\right)$ and $S * S * X_{-}$:

$$
\begin{aligned}
\Delta\left(X_{-}^{*}\right) & =-q \Delta X_{-}=-q\left(X_{-} \otimes K^{-1}+\mathbf{1} \otimes X_{-}\right) \\
& =X_{-}^{*} \otimes\left(K^{-1}\right)^{*}+\mathbf{1}^{*} \otimes X_{-}^{*} \\
& =\left(\Delta X_{-}\right)^{*}
\end{aligned}
$$

and

$$
\begin{aligned}
S * S * X_{-} & =S * S\left(-q X_{-}\right)=S *\left(-q S X_{-}\right)=S\left(*\left(q X_{-} K\right)\right)=S\left(q^{2} K^{*} X_{-}^{*}\right) \\
& =q^{2} S\left(K\left(-q X_{-}\right)=-S\left(X_{-}\right) S(K)=-\left(-X_{-} K K^{-1}\right)\right. \\
& =X_{-}
\end{aligned}
$$

Adding the cubic relations $x^{3}=\mathbb{1}, y^{3}=\mathbb{1}$ in $\mathcal{M}$, and the corresponding ones in the algebra $\mathcal{H}$, does not change anything to the determination of the star structures. This is because the (co)ideals are preserved by the involutions, and thus the quotients can still be done. 
Anyway, as $\mathcal{H}$ is dual to $\mathcal{F}$ (or $U_{q}(\operatorname{sl}(2))$ dual to $\operatorname{Fun}\left(S L_{q}(2, \mathbb{C})\right)$ ), we should have dual *structures. This means the relation

$$
<h^{*}, u>=\overline{<h,(S u)^{*}>}, \quad h \in \mathcal{H}, u \in \mathcal{F}
$$

holds. Moreover, the covariance condition for the star, equation (22), may also be written dually as a condition for $*$ under the action of $\mathcal{H}$. This can be done pairing the $\mathcal{F}$ component of equation (22) with some $h \in \mathcal{H}$. One should next recall the definition of the left action (18) (the right action if we are using the left coaction in (22)) and the duality (25) that relates both $*$-Hopf stars. One gets finally the constraint on $*_{\mathcal{M}}$ to be $\mathcal{H}$ covariant,

$$
h\left(z^{*}\right)=\left[(S h)^{*} z\right]^{*}, \quad h \in \mathcal{H}, z \in \mathcal{M} .
$$

Note that the $*$-operation that we obtain on the algebra $\mathcal{M}=M_{3}(\mathbb{C})$ does not coincide with the usual hermitian conjugacy ( $\dagger$-operation) on matrices, at least when $x$ and $y$ are represented with the $3 \times 3$ matrices given in Section 2 (they were such that $x^{\dagger}=x^{-1}=x^{2}$ and $y^{\dagger}=y^{-1}=y^{2}$ ). Moreover, it can be seen that it is not possible to find a star operation on the quantum group $\mathcal{F}$ that would respect the $\dagger$ involution on $\mathcal{M}$.

The situation here is reminiscent of the case where one introduces a "bar"-operation (Dirac conjugacy) on Dirac matrices (Clifford algebra for usual space-time) that is distinct from usual hermitian conjugacy. One remembers that, using this involution, one can build a scalar product on the space of spinors which is spin-invariant (the scalar product built using the usual hermitian conjugacy being positive but not invariant). The situation here is somewhat similar.

Remark: Note finally that one could be tempted to chose the involution defined by $K^{\star}=K^{-1}$, $X_{+}^{\star}= \pm X_{-}$and $X_{-}^{\star}= \pm X_{+}$. However, as it is easy to check (look for instance at the compatibility with the coproduct), this is a twisted star operation. This last star operation is the one one would need to interpret the unitary group of $\mathcal{H}$ as $U(3) \times U(2) \times U(1)$, which could be related to the gauge group of the Standard Model 12. Instead of using the standard Hopf-structure on $U_{q}(s l(2, \mathbb{C}))$ with this twisted star, a better sound mathematical alternative would be to look for a non-standard Hopf-structure for this quantum group (i.e., a different coproduct, but preserving the multiplication), such that $\star$ becomes a normal star operation.

\subsubsection{Matrix representation of the $*$-operation on $\mathcal{M}$}

The star operation for $\mathcal{M}$ introduced above $(* x=x, * y=y)$ can be written explicitly in terms of $3 \times 3$ matrices. Let us define the charge conjugation matrix $C$ :

$$
C \doteq\left(\begin{array}{lll}
1 & 0 & 0 \\
0 & 0 & 1 \\
0 & 1 & 0
\end{array}\right)
$$

For every element $m$ of the algebra $\mathcal{M}$ we set

$$
* m \doteq\left(C m C^{-1}\right)^{\dagger},
$$

where $\dagger$ denotes the usual hermitian conjugation (the transpose of the complex conjugate matrix). This is clearly a star operation: it is conjugate-linear, involutive $(*(* m)=m)$, anti-multiplicative $(*(m n)=(* n)(* m))$, and unital $(* \mathbb{1}=\mathbb{1})$. Remark also that $C=C^{-1}=C^{\dagger}$.

Let us represent the generators $x$ and $y$, as before, by $x=\left(\begin{array}{ccc}1 & 0 & 0 \\ 0 & q^{-1} & 0 \\ 0 & 0 & q^{-2}\end{array}\right)$ and $y=\left(\begin{array}{lll}0 & 1 & 0 \\ 0 & 0 & 1 \\ 1 & 0 & 0\end{array}\right)$. With (28) it is then easy to check that $* x=x, * y=y$. Therefore the star operation defined explicitly as before in terms of the charge conjugation matrix $C$ implements the abstract star operation introduced above. We already know that this star structure on $\mathcal{M}$ gives rise to star structures on the quantum group $\mathcal{F}$ and its dual $\mathcal{H}$ that are compatible with their Hopf algebra structure.

The most general self-adjoint element should satisfy $* m=m$, hence the vector space generators

$$
\mathbb{1}, x, y, x^{2}, q x y, y^{2}, q^{2} x^{2} y, q^{2} x y^{2}, q x^{2} y^{2}
$$


are "real" in the sense that they are self-adjoint for $*$. The same is obviously true for any linear combination of these generators with real coefficients. Using this, it is easy to write the most general $3 \times 3$ matrix that is self-adjoint for the star $*$. It can be written as:

$$
\alpha=\alpha_{i r r}+\alpha_{\text {eve }}+\alpha_{o d d},
$$

with

$$
\begin{aligned}
\alpha_{\text {irr }}= & \left(\begin{array}{ccc}
a_{20} & q a_{11} & a_{02} \\
a_{02} & q a_{20} & a_{11} \\
q^{2} a_{11} & a_{02} & q^{2} a_{20}
\end{array}\right)=a_{20} x^{2}+a_{11} q x y+a_{02} y^{2}, \\
\alpha_{\text {eve }}= & \left(\begin{array}{ccc}
a_{10} & a_{01} & q a_{22} \\
q^{2} a_{22} & q^{2} a_{10} & a_{01} \\
a_{01} & a_{22} & q a_{10}
\end{array}\right)=a_{10} x+a_{01} y+a_{22} q x^{2} y^{2}, \\
\alpha_{\text {odd }}= & \left(\begin{array}{ccc}
a_{00} & q^{2} a_{21} & q^{2} a_{12} \\
q a_{12} & a_{00} & a_{21} \\
q a_{21} & a_{12} & a_{00}
\end{array}\right)=a_{00} \mathbb{1}+a_{21} q^{2} x^{2} y+a_{12} q^{2} x y^{2},
\end{aligned}
$$

where all the $a_{r s}$ are arbitrary real numbers (warning: indices $r, s$ of $a_{r s}$ refer to the coefficients of $x^{r} y^{s}$ and not to the line or column indices of the corresponding matrix elements).

Notice that starting from the defining relations for the algebra $\mathcal{M}$ in terms of $x$ and $y$, and imposing $x^{*}=x, y^{*}=y$, it is not possible to find a matrix representation of this algebra for which these matrices would be $(\dagger$-)hermitian. Indeed such matrices would be in such a case diagonalisable with real eigenvalues, but the condition $x^{3}=y^{3}=\mathbb{1}$ would then imply $x=y=\mathbb{1}$.

\subsubsection{Inverses and unitary elements in $\mathcal{M}$}

To study the inverses of elements of $\mathcal{M}$, it is useful to define

$$
\begin{aligned}
C_{0}(t) & =\frac{1}{3}\left(\exp (t)+\exp (q t)+\exp \left(q^{2} t\right)\right) \\
C_{1}(t) & =\frac{d}{d t} C_{0}(t) \\
C_{2}(t) & =\frac{d}{d t} C_{1}(t)
\end{aligned}
$$

These functions appear as generalizations of sine and cosine functions when one replaces $\mathbb{Z}_{2}$ by $\mathbb{Z}_{3}$. They seem to have been invented several times in the literature (an old reference is [13). Call

$$
\begin{aligned}
& D(a, b, c)=a^{3}+b^{3}+c^{3}-3 a b c \\
& T(a, b, c)=a^{2}-b c
\end{aligned}
$$

In particular, it is easy to see that $D\left(C_{0}, C_{1}, C_{2}\right)=1$ (a generalization of $\left.\sin ^{2}+\cos ^{2}=1\right)$.

Writing $\mathcal{M}=3_{\text {irr }} \oplus 3_{\text {eve }} \oplus 3_{\text {odd }}$, it can be shown that (with obvious notations)

$$
\text { Inverse }\left(3_{\text {eve }}\right) \subset 3_{\text {irr }}, \quad \text { Inverse }\left(3_{\text {irr }}\right) \subset 3_{\text {eve }}, \quad \text { but Inverse }\left(3_{\text {odd }}\right) \subset 3_{\text {odd }} .
$$

More precisely, given an arbitrary element $a_{\text {odd }} \in 3_{\text {odd }}$, we have

$$
\begin{aligned}
& a_{\text {odd }}=a_{00} \mathbb{1}+a_{12} q^{2} x y^{2}+a_{21} q^{2} x^{2} y \\
& a_{\text {odd }}^{-1}=\frac{1}{D\left(a_{00}, a_{12}, a_{21}\right)}\left[T\left(a_{00}, a_{12}, a_{21}\right) \mathbb{1}+T\left(a_{21}, a_{00}, a_{12}\right) q^{2} x y^{2}+T\left(a_{12}, a_{21}, a_{00}\right) q^{2} x^{2} y\right] .
\end{aligned}
$$

Taking $a_{\text {eve }} \in 3_{\text {eve }}$,

$$
\begin{aligned}
& a_{\text {eve }}=a_{10} x+a_{01} y+a_{22} q x^{2} y^{2} \\
& a_{\text {eve }}^{-1}=\frac{1}{D\left(a_{10}, a_{01}, a_{22}\right)}\left[T\left(a_{10}, a_{01}, a_{22}\right) x^{2}+T\left(a_{22}, a_{10}, a_{01}\right) q x y+T\left(a_{01}, a_{22}, a_{10}\right) y^{2}\right] .
\end{aligned}
$$


Finally, for a general $a_{i r r} \in 3_{i r r}$, we see that

$$
\begin{aligned}
a_{i r r} & =a_{20} x^{2}+a_{11} q x y+a_{02} y^{2} \\
a_{i r r}^{-1} & =\frac{1}{D\left(a_{20}, a_{11}, a_{02}\right)}\left[T\left(a_{20}, a_{11}, a_{02}\right) x+T\left(a_{02}, a_{20}, a_{11}\right) y+T\left(a_{11}, a_{02}, a_{20}\right) q x^{2} y^{2}\right] .
\end{aligned}
$$

The result Inverse $\left(3_{\text {odd }}\right) \subset 3_{\text {odd }}$ should not be surprising, since $3_{\text {odd }}$ is an abelian subalgebra of $\mathcal{M}$ (the two other subspaces are not subalgebras); this algebra is actually isomorphic with the algebra $\mathbb{C}\left[\mathbb{Z}_{3}\right]$ of the abelian group $\mathbb{Z}_{3}$. Moreover, using this last isomorphism we can write

$$
\mathcal{M}=\mathbb{C}\left[\mathbb{Z}_{3}\right] \oplus x \mathbb{C}\left[\mathbb{Z}_{3}\right] \oplus x^{2} \mathbb{C}\left[\mathbb{Z}_{3}\right]=3_{\text {odd }} \oplus 3_{\text {eve }} \oplus 3_{\text {irr }}
$$

Remark that when the coefficients $a_{i j}$ are real, the generic elements $a_{o d d}, a_{\text {eve }}$ and $a_{i r r}$ written above are also real.

Since $3_{\text {odd }}$ is a subalgebra, it is interesting to consider the unitary elements within $3_{\text {odd }}$ (with respect to our star operation). A unitary element $\left(u^{*}=u^{-1}\right)$ can be written $u=\exp (i a)$, with $a^{*}=a$ ( $a$ is "real"). Therefore, working on $3_{\text {odd }}, u$ can be expanded as $u=\exp \left[i\left(a_{00} \mathbb{1}+a_{12} q^{2} x y^{2}+\right.\right.$ $\left.a_{21} q^{2} x^{2} y\right)$ ], where $a_{i j} \in \mathbb{R}$. Since $x^{2} y$ commutes with $x y^{2}$, the previous exponential can be written as a product of three exponentials, each with its own real parameter: $u=u_{0} u_{1} u_{2}$, with

$$
\begin{aligned}
& u_{0}=\exp \left(i a_{00} \mathbb{1}\right)=\exp \left(i a_{00}\right) \mathbb{1} \\
& u_{1}=\exp \left(i a_{12} q^{2} x y^{2}\right)=C_{0}\left(i a_{12}\right) \mathbb{1}+C_{1}\left(i a_{12}\right) q^{2} x^{2} y+C_{2}\left(i a_{12}\right) q^{2} x y^{2} \\
& u_{2}=\exp \left(i a_{21} q^{2} x^{2} y\right)=C_{0}\left(i a_{21}\right) \mathbb{1}+C_{1}\left(i a_{21}\right) q^{2} x^{2} y+C_{2}\left(i a_{21}\right) q^{2} x y^{2}
\end{aligned}
$$

\subsection{A quantum group invariant scalar product on the space of $3 \times 3$ matrices}

The usual scalar product on the space $\mathcal{M}=M_{3}(\mathbb{C})$ of $3 \times 3$ matrices is $m_{1}, m_{2} \rightarrow\left(m_{1}, m_{2}\right)=$ $\operatorname{Tr}\left(m_{1}^{\dagger} m_{2}\right)$. For every linear operator $\ell$ acting on $\mathcal{M}$, we can define the usual adjoint $\ell^{\dagger}$; however, this adjoint does not coincide with the star operation introduced previously. Our aim in this section is to find another scalar product better suited for our purpose.

We call $z, z^{\prime}$ two generic elements of $\mathcal{M}$ (linear combinations of $x^{r} y^{s}$ ), and $h$ a generic element of $\mathcal{H}$ (a linear combination of $X_{+}^{\alpha} K^{\beta} X_{-}^{\gamma}$ ). We know that the first ones act on $\mathcal{M}$ like multiplication operators and that the others act on $\mathcal{M}$ by twisted derivations or automorphisms. We also know the action of our star operation $*$ on these linear operators. We shall now obtain a unique scalar product by imposing that $*$ coincides with the adjoint associated with this scalar product. That is, we are asking for an inner product on $\mathcal{M}$ such that the (left) actions of $\mathcal{M}$ and $\mathcal{H}$ (each with its respective star) on that vector space may be thought as $*$-representations. Hence, for every linear operator $\ell$ acting on the nine-dimensional vector space $\mathcal{M}$, we impose:

$$
\left(z, \ell . z^{\prime}\right)=\left(\ell^{*} . z, z^{\prime}\right) .
$$

Moreover, $(\cdot, \cdot)$ should be hermitian, so that

$$
\left(z^{\prime}, z\right)=\overline{\left(z, z^{\prime}\right)} \text {. }
$$

As usual, $(\cdot, \cdot)$ is taken as antilinear with respect to the first argument and linear with respect to the second: $\left(\alpha z, \beta z^{\prime}\right)=\bar{\alpha} \beta\left(z, z^{\prime}\right)$, with $\alpha, \beta \in \mathbb{C}$.

Due to the fact that $\left(z, z^{\prime}\right)=\left(\mathbb{1}, z^{*} z^{\prime}\right)$, it is enough to compute $(\mathbb{1}, z)$ for all the $z$ belonging to $\mathcal{M}$. We now use the action of $\mathcal{H}$ in (29) and obtain

$$
\left(h^{*} . \mathbb{1}, z\right)=(\mathbb{1}, h . z) .
$$

Note that we are now calling $h . z$ the left action that was previously denoted with $h^{L}[z]$. Taking $h=K$ and $z=x^{r} y^{s}$ we see that (use the results of Section 4.4. Table 1):

$$
\left(\mathbb{1}, K \cdot x^{r} y^{s}\right)=q^{(r-s)}\left(\mathbb{1}, x^{r} y^{s}\right)
$$


but

$$
\left(\mathbb{1}, K . x^{r} y^{s}\right)=\left(K^{*} . \mathbb{1}, x^{r} y^{s}\right)=\left(K . \mathbb{1}, x^{r} y^{s}\right)=\left(\mathbb{1}, x^{r} y^{s}\right),
$$

and therefore we conclude that $\left(\mathbb{1}, x^{r} y^{s}\right)=0$ unless $r=s$. We have then to find $(\mathbb{1}, \mathbb{1}),(\mathbb{1}, x y)$ and $\left(\mathbb{1}, x^{2} y^{2}\right)$.

Taking $h=X_{+}$we find

$$
\begin{aligned}
\left(\mathbb{1}, X_{+} \cdot z\right) & =\left(X_{+}^{*} \cdot \mathbb{1}, z\right)=\left(-q^{2} X_{+} \cdot \mathbb{1}, z\right) \\
& =-q(0, z)=0
\end{aligned}
$$

With $z=x^{2} y$ we have $X_{+} \cdot x^{2} y=q^{2} \mathbb{1}$, so $(\mathbb{1}, \mathbb{1})=0$. Selecting $z=y^{2}$, we have $X_{+} \cdot y^{2}=-q^{2} x y$ and thus $(\mathbb{1}, x y)=0$.

Hence, the only non vanishing scalar product of the type $(\mathbb{1}, z)$ is $\left(\mathbb{1}, x^{2} y^{2}\right)$. From this quantity we deduce eight other non-zero scalar products $\left(z, z^{\prime}\right)$, where $z$ and $z^{\prime}$ are basis elements $x^{r} y^{s}$. For instance, $\left(x, x y^{2}\right)=\left(\mathbb{1}, x^{*} \cdot x y^{2}\right)=\left(\mathbb{1}, x^{2} y^{2}\right)$.

Hermiticity with respect to $*$ implies that $(x y, x y)$ should be real, indeed $(x y, x y)=\overline{(x y, x y)}$. We set

$$
(x y, x y)=1 .
$$

We should however remember that this normalization condition is arbitrary and could be set to any other positive real number (this comment may become physically relevant when one introduces a "coupling constant" playing a role in some physical model).

One can summarise the results in the formula:

$$
\left(x^{p} y^{t}, x^{r} y^{s}\right)=q^{2} \delta_{p+r, 2} \delta_{t+s, 2}\left[q^{2} \delta_{s, 0}+q \delta_{s, 1}+\delta_{s, 2}\right],
$$

where $\delta_{i, j}$ is the Kroneker delta. In the basis

$$
\left\{\left\{x^{2} y, x y^{2}, x^{2} y^{2}, y^{2}\right\},\left\{y, x, \mathbb{1}, x^{2}\right\},\{x y\}\right\}
$$

the $9 \times 9$ matrix of scalar products $\left(z, z^{\prime}\right)$ reads

$$
\left(\begin{array}{ccc}
0 & U & 0 \\
U^{\dagger} & 0 & 0 \\
0 & 0 & 1
\end{array}\right),
$$

where $U$ is the $4 \times 4$ diagonal matrix $\operatorname{Diag}(1, q, q, q)$. Notice that the trace and determinant of this matrix are equal to 1 . The total space splits (Witt's decomposition) into the sum of two complementary isotropic spaces of dimension 4 and a supplementary space of dimension 1.

The real part of this non degenerate hermitian scalar product is a bilinear form of signature $(5,4)$. More precisely, if one uses the basis:

$$
\begin{array}{ll}
u_{1}=-y+x^{2} y & u_{2}=y+x^{2} y \\
v_{1}=-1+x^{2} y^{2} & v_{2}=1+x^{2} y^{2} \\
w_{1}=-x+x y^{2} & w_{2}=x+x y^{2} \\
t_{1}=-x^{2}+y^{2} & t_{2}=x^{2}+y^{2} \\
s=x y &
\end{array}
$$

our hermitian form can be written

$$
\left(\begin{array}{ccccccccc}
-2 & 0 & 0 & 0 & 0 & 0 & 0 & 0 & 0 \\
0 & 2 & 0 & 0 & 0 & 0 & 0 & 0 & 0 \\
0 & 0 & 1 & q-q^{2} & 0 & 0 & 0 & 0 & 0 \\
0 & 0 & -q+q^{2} & -1 & 0 & 0 & 0 & 0 & 0 \\
0 & 0 & 0 & 0 & 1 & q-q^{2} & 0 & 0 & 0 \\
0 & 0 & 0 & 0 & -q+q^{2} & -1 & 0 & 0 & 0 \\
0 & 0 & 0 & 0 & 0 & 0 & 1 & q-q^{2} & 0 \\
0 & 0 & 0 & 0 & 0 & 0 & -q+q^{2} & -1 & 0 \\
0 & 0 & 0 & 0 & 0 & 0 & 0 & 0 & 1
\end{array}\right)
$$


Its real part is diagonal in this base (since $\bar{q}=q^{2}$ ), and reads

$$
\operatorname{Diag}(-2,2,1,-1,1,-1,1,-1,1)
$$

The signature $(5,4)$ can be read immediately from the above.

As we saw in Section 4.6, the algebra $\mathcal{M}$ can be written as the sum of three vector spaces of dimensionality 3 corresponding to the decomposition of the underlying vector space as the sum of three indecomposable representations of the algebra $\mathcal{H}: \mathcal{M}=3_{\text {irr }} \oplus 3_{\text {eve }} \oplus 3_{\text {odd }}$, where

$$
\begin{aligned}
& 3_{\text {irr }}=\operatorname{Span}\left(x^{2}, x y, y^{2}\right) \\
& 3_{\text {odd }}=\operatorname{Span}\left(\mathbb{1}, x^{2} y, x y^{2}\right) \\
& 3_{\text {eve }}=\operatorname{Span}\left(x, y, x^{2} y^{2}\right) .
\end{aligned}
$$

It is therefore interesting to write the scalar product in a basis adapted to this decomposition.

In the basis $\left\{\left\{x^{2}, x y, y^{2}\right\},\left\{x, y, x^{2} y^{2}\right\},\left\{\mathbb{1}, x^{2} y, x y^{2}\right\}\right\}$, the scalar product can be written as

$$
G=\left(\begin{array}{ccc}
B & 0 & 0 \\
0 & 0 & B \\
0 & B & 0
\end{array}\right)
$$

where $B$ is the $3 \times 3$ block

$$
B=\left(\begin{array}{ccc}
0 & 0 & q^{2} \\
0 & 1 & 0 \\
q & 0 & 0
\end{array}\right) .
$$

Using the charge conjugation matrix (27) to write the star as in (28), the scalar product on $\mathcal{M}$ can be written as

$$
\left(m_{1}, m_{2}\right)=\frac{1}{3} \operatorname{Tr}\left(B^{t} C m_{1}^{*} m_{2}\right)=\frac{1}{3} \operatorname{Tr}\left(B^{t} m_{1}^{\dagger} C m_{2}\right),
$$

where the trace is a usual trace on the matrices.

\subsubsection{Quantum group invariance of the scalar product}

We should now justify why the above scalar product was called a quantum group invariant one. Remember we only said the scalar product was such that the stars coincide with the adjointoperators, or such that the actions are given by $*$-representations.

We refer the reader to [14, where it is shown that the $*$-representation condition on the scalar product,

$$
(h . z, w)=\left(z, h^{*} . w\right), \quad h \in \mathcal{H},
$$

automatically fulfils one of the two alternative invariance conditions that can be imposed on the scalar product, namely

$$
\left(\left(S h_{1}\right)^{*} \cdot z, h_{2} \cdot w\right)=\epsilon(h)(z, w), \quad \text { with } \Delta h=h_{1} \otimes h_{2}
$$

As a side note, we can check the classical limit: let $H$ be the universal enveloping algebra of some Lie algebra, and $x$ any antihermitian generator of the Lie algebra (such that $\exp (x)$ is unitary). As the natural Hopf structure on this particular cocommutative $H$ is given by $\epsilon(x)=0, S(x)=-x$, and $\Delta x=x \otimes 1+1 \otimes x$, (33) reduces in this case to

$$
\begin{aligned}
((* S x) . z, 1 \cdot w)+((* S 1) . z, x \cdot w) & =(x \cdot z, w)+(z, x \cdot w) \\
& =0 .
\end{aligned}
$$

Note that this last equation is just what is needed to get the scalar product invariance in the usual sense:

$$
\begin{aligned}
\left(z^{\prime}, w^{\prime}\right) & \doteq(\exp (x) \cdot z, \exp (x) \cdot w) \\
& =((1+x) \cdot z,(1+x) \cdot w)+\mathcal{O}\left(x^{2}\right)=(z, w)+(x \cdot z, w)+(z, x \cdot w)+\mathcal{O}\left(x^{2}\right) \\
& =(z, w)
\end{aligned}
$$


The relations dual to (33) and (32) are those that apply to the coaction of $\mathcal{F}$ instead of the action of $\mathcal{H}$. These are

$$
\left(\Delta_{R} z, \Delta_{R} w\right)=(z, w) \mathbb{1}_{\mathcal{F}},
$$

where $\left(\Delta_{R} z, \Delta_{R} w\right)$ should be understood as $\left(z_{i}, w_{j}\right) T_{i}^{*} T_{j}$ if $\Delta_{R} z=z_{i} \otimes T_{i}$, etc., and

$$
\left(z, \Delta_{R} w\right)=\left((1 \otimes S) \Delta_{R} z, w\right),
$$

respectively. We have used here the right-coaction, but the formulas for the left coaction can be trivially deduced from the above ones.

It is worth noting that these equations for the coaction of $\mathcal{F}$ imply the previous ones for the action of $\mathcal{H}$, and are completely equivalent assuming non-degeneracy of the pairing $\langle\cdot, \cdot\rangle$. Moreover, (34) is a requirement analogous to the condition of classical invariance by a group element action.

If we select an orthonormal basis $\left\{z_{i}\right\}$ of $\mathcal{M}$ such that $\Delta_{R} z_{i}=z_{j} \otimes T_{i}^{j}$ with $\Delta T_{i}^{j}=T_{k}^{j} \otimes T_{i}^{k}$, (34) and (35) reduce to

$$
\left(T_{i}^{k}\right)^{*} T_{j}^{k}=\delta_{i j} \mathbb{1}_{\mathcal{F}}
$$

and

$$
S T_{i}^{j}=\left(T_{j}^{i}\right)^{*},
$$

respectively. This last equation is exactly what we use in the classical (Lie algebra) case: the unitarity condition for matrices (the antipode is the correct quantum generalization of the group inverse), that warrants the unitarity of the representation (in the sense of invariance of the scalar product).

Applying the above discussion to the invariant subspaces of the Hopf-algebra $\mathcal{H}$ we obtain on each one the most general invariant scalar products that we list in Appendix E. 


\section{The Manin dual $\mathcal{M}^{!}$of $\mathcal{M}$}

In order to find the Manin dual [8] of a given quadratic algebra $\mathcal{A}$ given by the relations $E_{i j} x^{i} x^{j}=0$ amongst its generators $\left\{x^{i}\right\}$, one has first to determine all the matrices $\mathcal{E}$ such that $\mathcal{E}_{i j} E_{i j}=$ $\operatorname{Tr}\left(\mathcal{E}^{t} E\right)=0$. The Manin dual $\mathcal{A}^{!}$of $\mathcal{A}$ is defined as a quadratic algebra with relations $\mathcal{E}_{i j} \xi^{j} \xi^{j}=0$. This is equivalent to say that the quadratic relations in $\mathcal{A}^{!}$are orthogonal to the relations in $\mathcal{A}$, with the pairing (or "scalar product") $<\xi^{i}, x^{j}>=\delta^{i j}$.

The quadratic relations defining $\mathcal{M}(x y=q y x)$ can be written $E_{i j} x^{i} x^{j}=0$, with $x^{1} \doteq x, x^{2} \doteq y$ and $E_{i j}=\left(\begin{array}{cc}0 & 1 \\ -q & 0\end{array}\right)$. Hence we have, in the present case, $\mathcal{E}^{t} E=\left(\begin{array}{ll}-q \mathcal{E}_{21} & \mathcal{E}_{11} \\ -q \mathcal{E}_{22} & \mathcal{E}_{12}\end{array}\right)$. Thus

$$
\operatorname{Tr}\left(\mathcal{E}^{t} E\right)=-q \mathcal{E}_{21}+\mathcal{E}_{12} \equiv 0 \leadsto \mathcal{E}_{12}=q \mathcal{E}_{21} .
$$

The matrices solution of our problem span therefore a vector space of dimension 3 :

$$
\mathcal{E}=\left(\begin{array}{cc}
\mathcal{E}_{11} & q \mathcal{E}_{21} \\
\mathcal{E}_{21} & \mathcal{E}_{22}
\end{array}\right)=\mathcal{E}_{11} \mathcal{E}^{(1)}+\mathcal{E}_{21} \mathcal{E}^{(2)}+\mathcal{E}_{22} \mathcal{E}^{(3)}
$$

with

$$
\mathcal{E}^{(1)}=\left(\begin{array}{ll}
1 & 0 \\
0 & 0
\end{array}\right), \quad \mathcal{E}^{(2)}=\left(\begin{array}{ll}
0 & q \\
1 & 0
\end{array}\right), \quad \mathcal{E}^{(3)}=\left(\begin{array}{ll}
0 & 0 \\
0 & 1
\end{array}\right) .
$$

Our algebra $\mathcal{M}$ is not quadratic since we impose the relations $x^{3}=y^{3}=\mathbb{1}$. Nevertheless, forgetting momentarily those constraints, we define its Manin dual $\mathcal{M}^{!}$as the algebra generated over the complex numbers by $\xi^{1}, \xi^{2}$, satisfying the relations

$$
\mathcal{E}_{i j}^{(\sigma)} \xi^{i} \xi^{j}=0, \quad \sigma \in\{1,2,3\}
$$

i.e.,

$$
\left(\xi^{1}\right)^{2}=0, \quad q \xi^{1} \xi^{2}+\xi^{2} \xi^{1}=0, \quad\left(\xi^{2}\right)^{2}=0 .
$$

This means, in particular, that any cubic polynomial in the $\xi$ 's is identically zero. There is no need to introduce additional constraints for $\xi^{1}, \xi^{2}$ orthogonal to the cubic relations in $\mathcal{M}$.

We shall write $d x \doteq \xi^{1}$ and $d y \doteq \xi^{2}$, so $\mathcal{M}^{\text {! }}$ is defined by these two generators and the relations

$$
d x^{2}=0, \quad d y^{2}=0, \quad q d x d y+d y d x=0 .
$$

\section{1 $\mathcal{F}$ coacting on $\mathcal{M}^{!}$}

Once the coaction of $\mathcal{F}$ on $\mathcal{M}$ has been defined as in Section 3, and once the quantum group product relations have been obtained, it is easy to check that the coaction of $\mathcal{F}$ on $\mathcal{M}$ ! is given by the same formulae as for $\mathcal{M}$ itself. Namely, writing

$$
\left(\begin{array}{l}
d x^{\prime} \\
d y^{\prime}
\end{array}\right)=\left(\begin{array}{ll}
a & b \\
c & d
\end{array}\right) \otimes\left(\begin{array}{l}
d x \\
d y
\end{array}\right)
$$

and

$$
\left(\begin{array}{ll}
\tilde{d} x & \tilde{d} y
\end{array}\right)=\left(\begin{array}{ll}
d x & d y
\end{array}\right) \otimes\left(\begin{array}{ll}
a & b \\
c & d
\end{array}\right)
$$

ensures that $q d x^{\prime} d y^{\prime}+d y^{\prime} d x^{\prime}=0$ and $q \tilde{d} x \tilde{d} y+\tilde{d} y \tilde{d} x=0$, given that the relation $q d x d y+d y d x=0$ is satisfied. Actually, one can also recover $F u n\left(S L_{q}(2, \mathbb{C})\right)$ imposing just the invariance of relations (11) and (36) under the quantum group left-coactions.

The coaction can be read from those formulae, for instance $\Delta_{R}(d x)=d x \otimes a+d y \otimes c$.

\section{2 $\mathcal{H}$ acting on $\mathcal{M}^{!}$}

Since the formulae for the (left or right) coactions are the same, the formulae for the actions of $\mathcal{H}$ on $\mathcal{M}$ and $\mathcal{M}^{!}$must also coincide. For instance, using $X_{-}^{L}[x]=y$ we find immediately $X_{-}^{L}[d x]=d y$. This corresponds to an irreducible two-dimensional representation of $\mathcal{H}$. Anyway, we shall return to this problem in the next section, since we are going to introduce a differential algebra $\Omega_{W Z}(\mathcal{M})$ built over $\mathcal{M}$ and investigate its contents in terms of representations of $\mathcal{H}$. 


\section{Covariant differential calculus on $\mathcal{M}$}

\subsection{Differential algebras associated with a given algebra}

Given an algebra $\mathcal{A}$, there is a universal construction that allows one to build the so-called algebra of universal differential forms $\Omega(\mathcal{A})=\sum_{p=0}^{\infty} \Omega^{p}(\mathcal{A})$ over $\mathcal{A}$. This differential algebra is universal, in the sense that any other differential algebra with $\Omega^{0}(\mathcal{A})=\mathcal{A}$ will be a quotient of the universal one. Schematically, the construction of $\Omega(\mathcal{A})$ goes as follows. Let $m$ be the multiplication map: $m(a \otimes b) \doteq a b$, for $a$ and $b$ in $\mathcal{A}$ and let $\operatorname{ker}(m)$ be the kernel of this map $(a \otimes b \in \operatorname{ker}(m)$ implies that $a b=0)$. One builds the algebra of universal forms as the graded vector space:

$$
\begin{aligned}
\Omega^{0}(\mathcal{A}) & \doteq \mathcal{A} \\
\Omega^{1}(\mathcal{A}) & \doteq \operatorname{ker}(m) \subset \mathcal{A} \otimes \mathcal{A} \\
\Omega^{p}(\mathcal{A}) & \doteq \Omega^{1}(\mathcal{A}) \otimes_{\mathcal{A}} \Omega^{1}(\mathcal{A}) \otimes_{\mathcal{A}} \ldots \otimes_{\mathcal{A}} \Omega^{1}(\mathcal{A})
\end{aligned}
$$

The above tensor products are taken over the algebra $\mathcal{A}$. Multiplication is defined as follows:

$$
\left(a_{0} \otimes a_{1} \otimes \ldots \otimes a_{n}\right)\left(b_{0} \otimes b_{1} \otimes \ldots \otimes b_{n}\right) \doteq\left(a_{0} \otimes a_{1} \otimes \ldots \otimes a_{n} b_{0} \otimes b_{1} \otimes \ldots b_{n}\right) .
$$

One also writes $a_{0} d a_{1} \doteq a_{0} \otimes a_{1}-a_{0} a_{1} \otimes \mathbb{1}$, etc. Notice that $d \mathbb{1}=0$. More details about this construction can be found, for instance, in [15].

In the present situation, $\mathcal{A}=\mathcal{M}$. Since $\Omega^{1}(\mathcal{M})=\operatorname{ker}(m)$, we find that $\operatorname{dim}\left(\Omega^{1}(\mathcal{M})\right)=$ $\operatorname{dim}(\mathcal{M} \otimes \mathcal{M})-\operatorname{dim}(\mathcal{M})=9^{2}-9=72$. The dimension of $\Omega(\mathcal{M})$ itself is infinite (notice that there are no particular relations between $d x$ and $d y$ so $\Omega^{p}(\mathcal{M})$ never vanishes).

For practical purposes, it is often not very convenient to work with the algebra of universal forms. First of all, it is very "big". Next, it does not remember anything of the coaction of $\mathcal{F}$ on the algebra $\mathcal{M}$ (the 0 -forms).

Starting from a given algebra, there are several constructions that allow one to build "smaller" differential calculi. As already mentioned, they will all be quotients of the algebra of universal forms by some (differentiable) ideal. One possibility for such a construction was described by [16], another one by [17], and yet another one by [18]. In the present case, however, we shall use something else, namely the differential calculus $\Omega_{W Z}$ introduced by Wess and Zumino [6] (see also (5) in the case of the quantum 2-plane. Its main interest, as we shall see below, is that it is covariant with respect to the action (or coaction) of a quantum group. This differential algebra is, as usual, a graded vector space, and our first step will be to define the differentials $d x$ and $d y$ together with their commutation relations.

\subsection{The Wess-Zumino complex}

The differential algebra $\Omega_{W Z}$ that we are going to use was introduced historically by [6], in the case of the quantum plane. It is only one of the many possible differential calculi that one can associate with this algebra, but it is the only one (up to trivial modifications) that is both quadratic and compatible with the coaction of the group $\operatorname{Fun}\left(S L_{q}(2, \mathbb{C})\right)$. Since it will play an important role in what follows, we shall briefly recall how it can be obtained.

First of all $\Omega_{W Z}=\Omega_{W Z}^{0} \oplus \Omega_{W Z}^{1} \oplus \Omega_{W Z}^{2}$ is a graded vector space.

- Forms of grade 0 just coincide with the functions on the quantum plane, i.e., they are polynomials in $x$ and $y$.

- Forms of grade 1 are of the type $a_{r s} x^{r} y^{s} d x+b_{r s} x^{r} y^{s} d y$, where $d x$ and $d y$ are those introduced in Section 6, devoted to the construction of the Manin dual $\mathcal{M}^{\text {! }}$. The Manin dual is the subalgebra $\left\{\lambda_{00}+\lambda_{10} d x+\lambda_{01} d y+\lambda_{11} d x d y\right\}$ of $\Omega_{W Z}$, where the coefficients $\lambda_{i j}$ belong to the field of scalars.

- Forms of grade 2 are of the type $c_{r s} x^{r} y^{s} d x d y$.

Next, $\Omega_{W Z}$ is an algebra. We need therefore to find the relations between $x, y, d x$ and $d y$. Moreover, we want $\Omega_{W Z}$ to be a differential algebra, so we set $d(x)=d x, d(y)=d y$ and the Leibniz rule (for $d$ ) should hold. Also, we set $d \mathbb{1}=0$ and $d^{2}=0$. 
Assuming quadraticity of the algebra, we expand a priori $x d x, x d y, y d x$ and $y d y$ in terms of $d x x, d y x, d x y$ and $d y y$. This involves sixteen unknown coefficients. Applying $d$ to the above, we get four relations between these coefficients. We are left with twelve independent parameters.

Differentiating the relation $x y-q y x=0$ and replacing $x d x, x d y, y d x$ and $y d y$ by what was postulated before gives one identity that fixes three of the unknown coefficients (actually one finds four constraints but one of them is not independent of the others). We are left with $12-3=9$ independent parameters.

Next, one uses compatibility with the (left or right) coaction of $\operatorname{Fun}\left(S L_{q}(2, \mathbb{C})\right.$ ), i.e., one writes $x^{\prime}=a \otimes x+b \otimes y, y^{\prime}=c \otimes x+d \otimes y, d x^{\prime}=a \otimes d x+b \otimes d y, d y^{\prime}=c \otimes d x+d \otimes d y$ and imposes that the relations 7 between $x^{\prime}, y^{\prime}$ and $d x^{\prime}, d y^{\prime}$ are the same as the relations between the corresponding untransformed elements (the unprimed ones). In this way one obtains four identities that fix 8 additional parameters. One is left with just one $(=9-8)$ free parameter.

The last parameter is fixed by checking associativity of the cubics, for instance $(x d y) d x$ should be equal to $x(d y d x)$. One finds that this last parameter should be either equal to $q$ or to $1 / q$.

The final result is as follows:

$$
\begin{aligned}
& x y=q y x \\
& x d x=q^{2} d x x \quad x d y=q d y x+\left(q^{2}-1\right) d x y \\
& y d x=q d x y \quad y d y=q^{2} d y y \\
& d x^{2}=0 \quad d y^{2}=0 \\
& d x d y+q^{2} d y d x=0
\end{aligned}
$$

It may be useful to notice that $d y x=(q-1) y d x+q^{2} x d y$.

\subsection{A reduced Wess-Zumino complex}

In the case $q^{3}=1$, we add the following to the defining relation of the quantum plane: $x^{3}=\mathbb{1}, y^{3}=$ 1. As we know, this defines the algebra $\mathcal{M}$ (the space of $3 \times 3$ complex matrices) as a quotient of the quantum plane. Adding the same two cubic relations to the differential algebra $\Omega_{W Z}$ defines a differential algebra that we shall denote $\Omega_{W Z}(\mathcal{M})$. The fact that it is well defined as a differential algebra is not totally obvious and requires some checking. Technically, one has to verify that we are taking the quotient by a differential ideal. In plain terms, one has to check that $d\left(x^{3}\right)=d \mathbb{1}=0$ and that $d\left(y^{3}\right)=d \mathbb{1}=0$. This is indeed so:

$$
\begin{aligned}
& d\left(x^{3}\right)=d\left(x^{2}\right) x+x^{2} d x=(d x) x^{2}+x(d x) x+x^{2} d x=\left(1+q+q^{2}\right) x^{2} d x=0 \\
& d\left(y^{3}\right)=d\left(y^{2}\right) y+y^{2} d y=(d y) y^{2}+y(d y) y+y^{2} d y=\left(1+q+q^{2}\right) y^{2} d y=0
\end{aligned}
$$

Note that $\operatorname{dim}\left(\Omega_{W Z}^{0}\right)=9, \operatorname{dim}\left(\Omega_{W Z}^{1}\right)=9+9=18$ and $\operatorname{dim}\left(\Omega_{W Z}^{2}\right)=9$.

\subsection{The differential of an element of $\mathcal{M}$}

Let $m$ be an arbitrary $3 \times 3$ matrix. Let us call $m_{i j}, i, j \in\{1,2,3\}$ the matrix elements of $m$. By using elementary matrices, we know how to decompose $m$ on the basis $x^{r} y^{s}$ (see Section 2). This way we can write $m=m_{r s} x^{r} y^{s}$ (where, of course, the coefficients $m_{r s}$ do not coincide at all with the $m_{i j}$ !). It is then straightforward to compute the one-form $d m$ belonging to the reduced WessZumino differential algebra $\Omega_{W Z}(\mathcal{M})$ by using the Leibniz rule together with the commutation relations given previously. If we write

$$
d m=(d m)_{x} d x+(d m)_{y} d y
$$

\footnotetext{
${ }^{7}$ Observe that asking for $x y=q y x$ and $d x d y=-q^{-1} d y d x$ to be preserved under the coaction provides an alternative way of defining the quantum group. Here we are just refering to relations that mix one of $\{x, y\}$ with one of $\{d x, d y\}$.
} 
we obtain (here the indices are of type $i, j$, i.e., refer to the matrix elements of $m$ ):

$$
\begin{aligned}
d m= & \frac{1}{3}\left(\begin{array}{ccc}
\left(m_{11}-m_{33}\right)\left(1-q^{2}\right) & \left(m_{12}-m_{31}\right)\left(q^{2}-q\right) & \left(m_{32}-m_{13}\right)(1-q) \\
\left(m_{21}-m_{13}\right)\left(q^{2}-q\right) & \left(m_{11}-m_{22}\right)(1-q) & \left(m_{23}-m_{12}\right)\left(1-q^{2}\right) \\
\left(m_{23}-m_{31}\right)(1-q) & \left(m_{32}-m_{21}\right)\left(1-q^{2}\right) & \left(m_{33}-m_{22}\right)\left(q^{2}-q\right)
\end{array}\right) d x \\
& +\left(\begin{array}{ccc}
m_{12} & -m_{13} q^{2} & 0 \\
0 & m_{23} & -m_{21} q^{2} \\
-m_{32} q^{2} & 0 & m_{31}
\end{array}\right) d y
\end{aligned}
$$

Notice that differences of cubic roots of 1 appear in the matrix elements of $(d m)_{x}$.

\subsection{The action of $\mathcal{H}$ on $\Omega_{W Z}(\mathcal{M})$}

\subsubsection{The action of $\mathcal{H}$ on $\Omega_{W Z}^{0}(\mathcal{M})=\mathcal{M}$}

We already saw that the nine-dimensional space $\mathcal{M}$ can be decomposed into three indecomposable representations of $\mathcal{H}$ called $3_{\text {irr }}, 3_{\text {eve }}$ and $3_{\text {odd }}$. The $3_{\text {irr }}$ is irreducible and spanned by $x^{2}, x y, y^{2}$. The $3_{\text {eve }}$ is reducible indecomposable and spanned by $x, y, x^{2} y^{2}$; it contains an invariant 2 -dimensional subspace spanned by $x, y$. The $3_{o d d}$ is also reducible indecomposable and spanned by $\mathbb{1}, x^{2} y, x y^{2}$; it contains an invariant 1 -dimensional subspace spanned by $\mathbb{1}$.

\subsubsection{The action of $\mathcal{H}$ on $\Omega_{W Z}^{1}(\mathcal{M})$}

Since $\Omega_{W Z}^{1}(\mathcal{M})=\mathcal{M} d x \oplus \mathcal{M} d y$, and as $\{d x, d y\}$ span the two-dimensional irreducible representation of $\mathcal{H}$, it is a priori clear that we should decompose $3_{\text {irr }} \otimes 2,3_{\text {eve }} \otimes 2$ and $3_{\text {odd }} \otimes 2$ in indecomposable representations of $\mathcal{H}$. The action of elements $X \in \mathcal{H}$ on one-forms is obtained, as usual, from the coproduct rule; for instance,

$$
X_{+}^{L}[y d y]=X_{+}^{L}[y] \mathbb{1}[d y]+K^{L}[y] X_{+}^{L}[d y]=x d y+q^{2} y d x .
$$

In this way we obtain the following results (we only give the tables associated with the left action):

- The case $3_{\text {odd }} \otimes 2=3_{\text {irr }} \oplus 3_{\text {eve }}$.

\begin{tabular}{l||ccc} 
& $K^{L}$ & $X_{+}^{L}$ & $X_{-}^{L}$ \\
\hline \hline $\mathbb{1} d x$ & $q d x$ & 0 & $d y$ \\
$x^{2} y d x$ & $q^{2} x^{2} y d x$ & $q^{2} d x$ & $-q^{2} x y^{2} d x+x^{2} y d y$ \\
$x y^{2} d x$ & $x y^{2} d x$ & $-x^{2} y d x$ & $q d x+x y^{2} d y$ \\
\hline $\mathbb{1} d y$ & $q^{2} d y$ & $d x$ & 0 \\
$x^{2} y d y$ & $x^{2} y d y$ & $q^{2} d y+q x^{2} y d x$ & $-q x y^{2} d y$ \\
$x y^{2} d y$ & $q x y^{2} d y$ & $-x^{2} y d y+q^{2} x y^{2} d x$ & $d y$
\end{tabular}

This gives $3_{\text {irr }} \oplus 3_{\text {eve }}$, since, up to multiplicative factors (dashed arrows stand for the action of $X_{-}^{L}$ and continuous ones for $X_{+}^{L}$ )
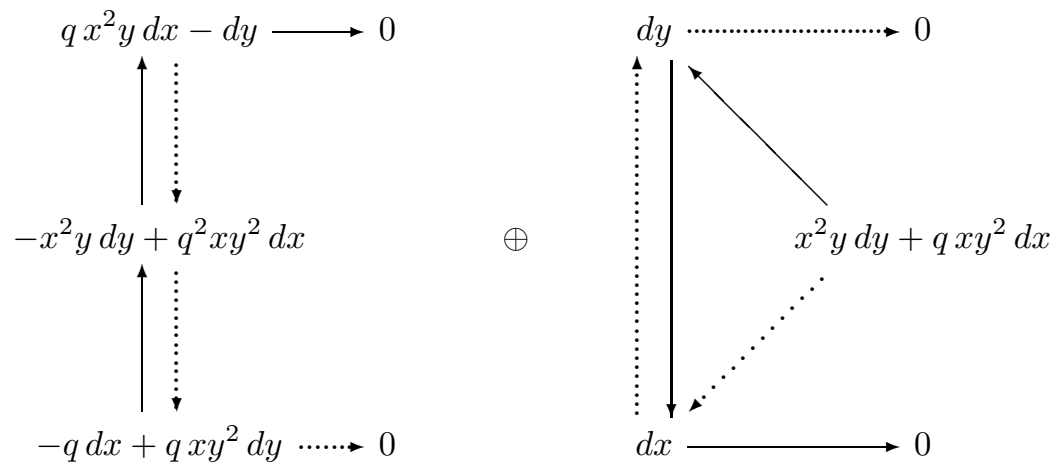

Note that $3_{\text {eve }}$ contains an irreducible 2-dimensional representation spanned by $\{d x, d y\}$. 
- The case $3_{\text {eve }} \otimes 2=3_{\text {irr }} \oplus 3_{\text {odd }}$.

\begin{tabular}{l||ccc} 
& $K^{L}$ & $X_{+}^{L}$ & $X_{-}^{L}$ \\
\hline \hline$x d x$ & $q^{2} x d x$ & 0 & $q^{2} y d x+x d y$ \\
$y d x$ & $y d x$ & $x d x$ & $y d y$ \\
$x^{2} y^{2} d x$ & $q x^{2} y^{2} d x$ & $-q y d x$ & $-x d x+x^{2} y^{2} d y$ \\
\hline$x d y$ & $x d y$ & $q x d x$ & $q y d y$ \\
$y d y$ & $q y d y$ & $x d y+q^{2} y d x$ & 0 \\
$x^{2} y^{2} d y$ & $q^{2} x^{2} y^{2} d y$ & $-q y d y+x^{2} y^{2} d x$ & $-q^{2} x d y$
\end{tabular}

This corresponds to $3_{i r r} \oplus 3_{\text {odd }}$, since, up to multiplicative factors,
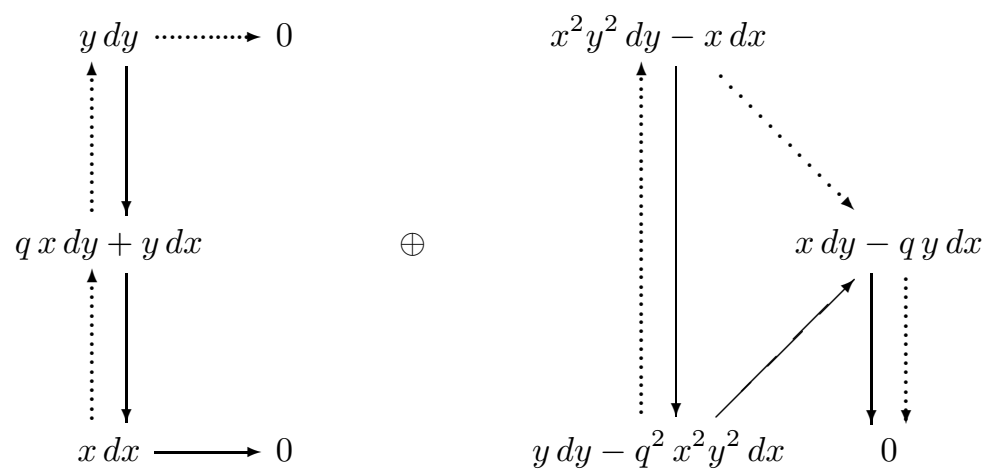

Remark that $3_{\text {odd }}$ contains an irreducible 1-dimensional representation spanned by $x d y-$ $q y d x$.

- The case $3_{\text {irr }} \otimes 2=6_{\text {eve }}$.

\begin{tabular}{c||ccc} 
& $K^{L}$ & $X_{+}^{L}$ & $X_{-}^{L}$ \\
\hline \hline$x^{2} d x$ & $x^{2} d x$ & 0 & $-q x y d x+x^{2} d y$ \\
$x y d x$ & $q x y d x$ & $q x^{2} d x$ & $y^{2} d x+x y d y$ \\
$y^{2} d x$ & $q^{2} y^{2} d x$ & $-q^{2} x y d x$ & $y^{2} d y$ \\
\hline$x^{2} d y$ & $q x^{2} d y$ & $q^{2} x^{2} d x$ & $-x y d y$ \\
$x y d y$ & $q^{2} x y d y$ & $q x^{2} d y+x y d x$ & $q^{2} y^{2} d y$ \\
$y^{2} d y$ & $y^{2} d y$ & $-q^{2} x y d y+q y^{2} d x$ & 0
\end{tabular}

This actually gives the six-dimensional indecomposable representation $6_{\text {eve }}$ (which is projective, cf. Appendix D. . It contains the four-dimensional indecomposable representation 4 eve, a family of indecomposables of the type $3_{e}^{\lambda}$, and one irreducible of dimension two, spanned by $\left\{-q^{2} x^{2} d y+x y d x,-q x y d y+y^{2} d x\right\}$. 


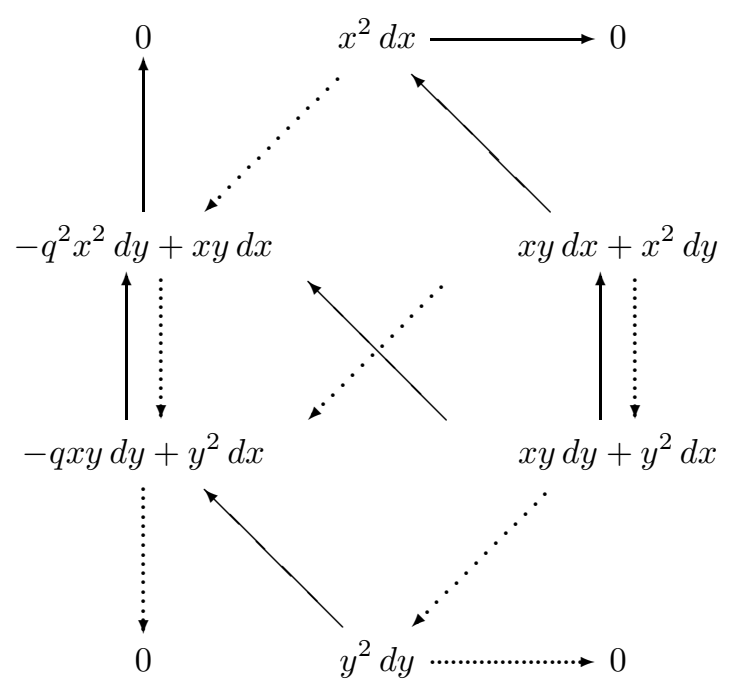

\subsubsection{The action of $\mathcal{H}$ on $\Omega_{W Z}^{2}(\mathcal{M})$}

The differential 2-form $d x d y$ that generates (over $\mathcal{M}) \Omega_{W Z}^{2}(\mathcal{M})$ is $\mathcal{H}$-invariant, since $X_{+}^{L}[d x d y]=$ $X_{-}^{L}[d x d y]=0$ and $K^{L}[d x d y]=d x d y$. Hence $\Omega_{W Z}^{2}(\mathcal{M})=\mathcal{M} d x d y$ has exactly the same decomposition in representations of $\mathcal{H}$ as $\mathcal{M}$.

\subsection{Cohomology of $d$ on $\Omega_{W Z}(\mathcal{M})$ and $\mathcal{H}$-representations}

We will now analyse the action of the differential operator $d$ on each level of the differential complex, as given by a basis adapted to the action of the quantum group $\mathcal{H}$. As usual, we shall call $\mathcal{Z}^{p} \doteq$ $\left\{\omega \in \Omega^{p}\right.$ with $\left.d \omega=0\right\}$, the space of $p$-cocycles and $\mathcal{B}^{p} \doteq\left\{\omega \in \Omega^{p}\right.$ such that $\omega=d \phi$, for $\left.\phi \in \Omega^{p-1}\right\}$, the space of $p$-coboundaries. Of course, $\mathcal{B}^{p} \subset \mathcal{Z}^{p}$, and we set $H^{p} \doteq \mathcal{Z}^{p} / \mathcal{B}^{p}$.

\subsubsection{Action of $d$ on $\Omega_{W Z}^{0}(\mathcal{M})$}

The differential operator $d$ acts on a basis of $\mathcal{M}$ as shown in the following table. The range and image vectors are classified according to the $\mathcal{H}$-representation to which they belong.

\begin{tabular}{l|ccr|c} 
& \multicolumn{3}{|c|}{$d$} & \\
\hline \hline \multirow{3}{*}{$3_{\text {odd }}$} & $\mathbb{1}$ & $\rightarrow$ & 0 & \multirow{2}{*}{2} \\
\cline { 2 - 4 } & $x^{2} y$ & $\rightarrow$ & $-q x y d x+x^{2} d y$ & 2 \\
& $x y^{2}$ & $\rightarrow$ & $q y^{2} d x-q^{2} x y d y$ & \\
\hline \hline \multirow{3}{*}{$3_{\text {eve }}$} & $x$ & $\rightarrow$ & $d x$ & \multirow{2}{*}{$3_{\text {eve }}$} \\
& $y$ & $\rightarrow$ & $d y$ & \\
\cline { 2 - 4 } & $x^{2} y^{2}$ & $\rightarrow$ & $-x y^{2} d x-q^{2} x^{2} y d y$ & \\
\hline \multirow{3}{*}{$3_{\text {irr }}$} & $x^{2}$ & $\rightarrow$ & $-q^{2} x d x$ & \multirow{2}{*}{$3_{\text {irr }}$} \\
& $x y$ & $\rightarrow$ & $q^{2} y d x+x d y$ & \\
& $y^{2}$ & $\rightarrow$ & $-q^{2} y d y$ &
\end{tabular}

As it should ( $d$ was built as a covariant differential operator), $d$ preserves the representations, mapping $3_{\text {eve }} \mapsto 3_{\text {eve }}, 3_{\text {irr }} \mapsto 3_{\text {irr }}$, and $3_{\text {odd }} \mapsto$ the quotient $3_{\text {odd }} / 1=2$. 


\subsubsection{Action of $d$ on $\Omega_{W Z}^{1}(\mathcal{M})$}

Dividing the space according to representations of $\mathcal{H}, d$ acts as follows:

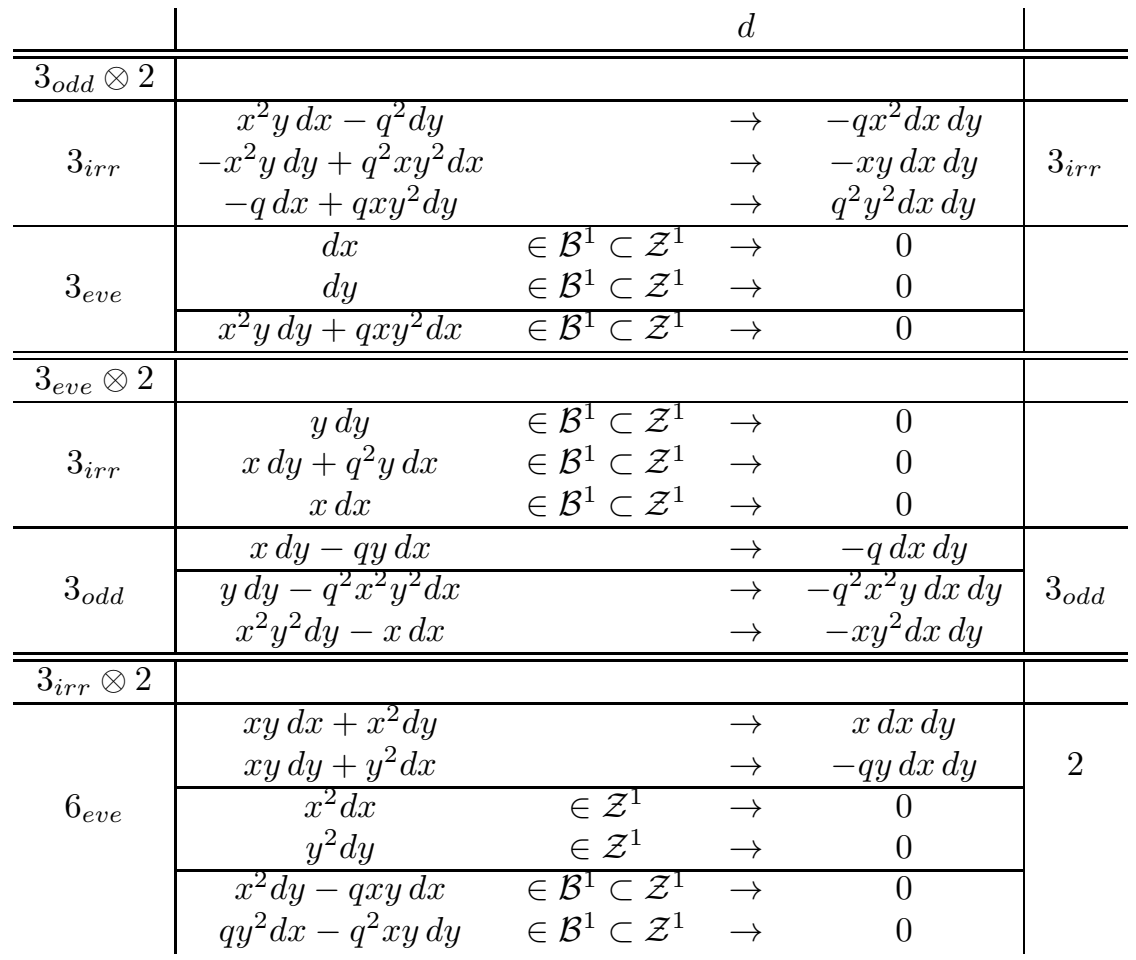

\subsubsection{Action of $d$ on $\Omega_{W Z}^{2}(\mathcal{M})$}

Here $d$ gives trivially 0 on all the elements, because there is no $\Omega^{3}$ subspace in our differential complex; however, one should distinguish between exact and closed forms:

$$
\begin{aligned}
3_{\text {odd }}: & \left\{d x d y, x^{2} y d x d y, x y^{2} d x d y\right\} \in \mathcal{B}^{2} \subset \mathcal{Z}^{2} \\
3_{\text {eve }}: & \{x d x d y, y d x d y\} \in \mathcal{B}^{2} \subset \mathcal{Z}^{2} \\
& \left\{x^{2} y^{2} d x d y\right\} \in \mathcal{Z}^{2} \\
3_{\text {irr }}: & \left\{x^{2} d x d y, x y d x d y, y^{2} d x d y\right\} \in \mathcal{B}^{2} \subset \mathcal{Z}^{2}
\end{aligned}
$$

\subsubsection{Cohomology of $d$}

From the previous tables, we see that

$$
\begin{array}{lll}
\operatorname{dim}\left(\mathcal{Z}^{0}\right)=1, & \operatorname{dim}\left(\mathcal{B}^{0}\right)=0, & \text { hence } \operatorname{dim}\left(H^{0}\right)=1 \\
\operatorname{dim}\left(\mathcal{Z}^{1}\right)=10, & \operatorname{dim}\left(\mathcal{B}^{1}\right)=8, & \text { hence } \operatorname{dim}\left(H^{1}\right)=2, \\
\operatorname{dim}\left(\mathcal{Z}^{2}\right)=9, & \operatorname{dim}\left(\mathcal{B}^{2}\right)=8, & \text { hence } \operatorname{dim}\left(H^{2}\right)=1
\end{array}
$$

Remark that $\chi=\operatorname{dim}\left(H^{0}\right)-\operatorname{dim}\left(H^{1}\right)+\operatorname{dim}\left(H^{2}\right)=1-2+1=0$.

\section{7 $\quad$ Star operations on the differential calculus $\Omega_{W Z}(\mathcal{M})$}

Given the $*$ operation on the algebra $\mathcal{M}$, we want to extend it to the differential algebra $\Omega_{W Z}(\mathcal{M})$. As usual, it has to be involutive, anti-multiplicative for the algebra structure in $\Omega_{W Z}(\mathcal{M})$, and complex sesquilinear. Moreover, it should be compatible with the coaction of $\mathcal{F}$. However, there is no reason a priori to impose that $*$ should commute with $d$. 
The quantum group covariance condition is, again, just the commutativity of the $*, \Delta_{R, L}$ diagram, or, algebraicaly,

$$
\left(\Delta_{R, L} \omega\right)^{*}=\Delta_{R, L}\left(\omega^{*}\right)
$$

In any case, it is enough to determine the action of $*$ on the generators $d x$ and $d y$, since we already determined the $*$ operation on $\mathcal{M}(* x=x, * y=y)$.

Taking $\Delta_{L} d x=a \otimes d x+b \otimes d y$, we get $\left(\Delta_{L} d x\right)^{*}=a \otimes d x^{*}+b \otimes d y^{*}$, to be compared with $\Delta_{L}\left(d x^{*}\right)$. It is trivial that the solution $d x^{*}=d x$ is a permissible one. But it is also the only one, up to complex phases. To see this one should expand $d x^{*}$ as a generic element of $\Omega_{W Z}^{1}(\mathcal{M})($ we want a grade-preserving $*)$ in the previous condition. Solving for the coefficients, and adding the requirement $x^{*} d x^{*}=q d x^{*} x^{*}$ (for instance, it is the $*$ of $d x x=q^{2} x d x$ ), we see that the result is:

$$
d x^{*}=d x, \quad d y^{*}=d y .
$$

Remark: On $\Omega_{W Z}, d$ is a graded derivation. First, $*$ is defined on $\Omega_{W Z}^{0}=\mathcal{M}$. It is also defined on the $d$ of the generators of $\mathcal{M}$ (in our case $* d x=d x$ and $* d y=d y$ ). Then $*$ is extended to the whole of the differential algebra $\Omega_{W Z}$ by imposing the anti-multiplicative property $*\left(\omega_{1} \omega_{2}\right)=\left(* \omega_{2}\right)\left(* \omega_{1}\right)$. Let $\phi=a_{i} d x^{i}$ be an arbitrary element of $\Omega_{W Z}^{1}$. Then $d\left(a_{i} d x^{i}\right)=d\left(a_{i}\right) d x^{i}$, SO

$$
* d\left(a_{i} d x^{i}\right)=\left(d a_{i} d x^{i}\right)^{*}=\left(d x^{i}\right)^{*}\left(d a_{i}\right)^{*}=d x^{i}\left(d a_{i}\right)^{*} .
$$

But, at the same time,

$$
d *\left(a_{i} d x^{i}\right)=d\left(\left(d x^{i}\right)^{*}\left(a_{i}\right)^{*}\right)=d\left(d x^{i}\left(a_{i}\right)^{*}\right)=-d x^{i} d\left(a_{i}^{*}\right) .
$$

In the case of a (real) manifold, we have an almost trivial star, with $* a_{i}=a_{i}$ and $* d a_{i}=d a_{i}$ for $a_{i} \in \Omega_{W Z}^{0}$, so $d * a_{i}=d a_{i}$ and one finds $8 * d \phi=-d * \phi$, when $\phi \in \Omega_{W Z}^{1}$. One recovers the fact that $*$ is almost trivial, "almost" since it is still antimultiplicative: $*(d x d y)=d y d x$.

In our case the conclusion is the same, indeed, it can be checked that, for all elements $a_{i}$ in $\mathcal{M}$, one has $*\left(d a_{i}\right)=d\left(* a_{i}\right)$. Therefore (with $\left.\phi=a_{i} d x^{i} \in \Omega_{W Z}^{1}\right), d * \phi=-* d \phi$. More generally,

$$
d * \omega=(-1)^{p} * d \omega \quad \text { when } \quad \omega \in \Omega_{W Z}^{p} .
$$

A general expression for the structure of the most general hermitian one-forms will be given later.

Warning: we are not saying that the above involution is the only one that one can define on the Wess-Zumino complex. For instance, one could very well try to extend the involution $\dagger$ (the one for which $x^{\dagger}=x^{2}$ ) to this differential algebra. Such an extension may be possible, but it would not be compatible with the coaction of $\mathcal{F}$ (it would only be compatible with dilations of $x$ and $y$ by numerical scaling factors). Loosing the compatibility with the coaction of $\mathcal{F}$ is however clearly inacceptable since the main interest of the differential complex of Wess-Zumino rests on the fact that it is compatible with the coaction (indeed, one can construct many other differential algebras over $\mathcal{M}$ that are not compatible with the coaction of $\mathcal{F}$ !).

\subsection{Multiplicative properties of $\Omega_{W Z}(\mathcal{M})$ and $\mathcal{H}$-representations}

We now analyze the behaviour - as representations of $\mathcal{H}$ - of the product in $\Omega_{W Z}(\mathcal{M})$. Some facts are trivially obtained:

$$
\begin{aligned}
& \Omega_{W Z}^{2}(\mathcal{M}) \cdot \Omega_{W Z}^{2}(\mathcal{M})=0 \\
& \Omega_{W Z}^{1}(\mathcal{M}) \cdot \Omega_{W Z}^{2}(\mathcal{M})=\Omega_{W Z}^{2}(\mathcal{M}) \cdot \Omega_{W Z}^{1}(\mathcal{M})=0
\end{aligned}
$$

Moreover, as $d x d y$ is $\mathcal{H}$-invariant we know that $\Omega_{W Z}^{2}(\mathcal{M})$ is isomorphic to $\mathcal{M}$. Using this, and the fact that $d x d y$ is $\mathcal{M}$-central, we see that the products $\Omega^{2} \cdot \Omega^{0}$ and $\Omega^{0} \cdot \Omega^{2}$ can be understood in terms of products in $\mathcal{M}$.

\footnotetext{
8 This shows in particular that usual differential forms on a real manifold (forms that are real in the naive sense) cannot be "real" for the star operation. Indeed, for a one-form, $* \phi=\phi$ implies $* d \phi=-d \phi$. This peculiar aspect of usual differential forms is a general feature of any differential algebra and can only be circumvented at the expense of introducing graded star operations. We do not use such graded star operations in the present paper.
} 
Anyway, the multiplication table for representations in $\mathcal{M}$ is very easy to write down (for instance using the grading of the generators):

\begin{tabular}{c|ccc}
$\mathcal{M} \cdot \mathcal{M}$ & $3_{\text {odd }}$ & $3_{\text {eve }}$ & $3_{\text {irr }}$ \\
\hline \hline $3_{\text {odd }}$ & $3_{\text {odd }}$ & $3_{\text {eve }}$ & $3_{\text {irr }}$ \\
$3_{\text {eve }}$ & $3_{\text {eve }}$ & $3_{\text {irr }}$ & $3_{\text {odd }}$ \\
$3_{\text {irr }}$ & $3_{\text {irr }}$ & $3_{\text {odd }}$ & $3_{\text {eve }}$
\end{tabular}

Next we summarize the product relations amongst elements of $\Omega_{W Z}^{1}(\mathcal{M})$ :

\begin{tabular}{l|ccc}
$\Omega^{1}(\mathcal{M}) \cdot \Omega^{1}(\mathcal{M})$ & $3_{\text {odd }} \otimes 2$ & $3_{\text {eve }} \otimes 2$ & $3_{\text {irr }} \otimes 2=6_{\text {eve }}$ \\
\hline \hline $3_{\text {odd }} \otimes 2$ & $3_{\text {odd }}$ & $3_{\text {eve }}$ & $3_{\text {irr }}$ \\
$3_{\text {eve }} \otimes 2$ & $3_{\text {eve }}$ & $3_{\text {irr }}$ & $3_{\text {odd }}$ \\
$3_{\text {irr }} \otimes 2=6_{\text {eve }}$ & $3_{\text {irr }}$ & $3_{\text {odd }}$ & $3_{\text {eve }}$
\end{tabular}

Finally, here is the corresponding table for products between elements of $\mathcal{M}$ in a given representation and elements of $\Omega_{W Z}^{1}(\mathcal{M})$, also with fixed transformation properties:

\begin{tabular}{c|ccc}
$\mathcal{M} \cdot \Omega^{1}(\mathcal{M})$ or $\Omega^{1}(\mathcal{M}) \cdot \mathcal{M}$ & $3_{\text {odd }} \otimes 2$ & $3_{\text {eve }} \otimes 2$ & $3_{\text {irr }} \otimes 2=6_{\text {eve }}$ \\
\hline \hline $3_{\text {odd }}$ & $3_{\text {odd }} \otimes 2$ & $3_{\text {eve }} \otimes 2$ & $3_{\text {irr }} \otimes 2$ \\
$3_{\text {eve }}$ & $3_{\text {eve }} \otimes 2$ & $3_{\text {irr }} \otimes 2$ & $3_{\text {odd }} \otimes 2$ \\
$3_{\text {irr }}$ & $3_{\text {irr }} \otimes 2$ & $3_{\text {odd }} \otimes 2$ & $3_{\text {eve }} \otimes 2$
\end{tabular}




\section{Noncommutative generalized connections on $\mathcal{M}$ and their curvature}

\subsection{Generalized connections in non commutative geometry}

Let $\Omega$ be a differential calculus over a unital associative algebra $\mathcal{A}$, i.e., a graded differential algebra with $\Omega^{0}=\mathcal{A}$. Let $\mathcal{A}^{\prime}$ be a right module over $\mathcal{A}$. A covariant differential $\nabla$ on $\mathcal{A}^{\prime}$ is a map $\mathcal{A}^{\prime} \otimes_{\mathcal{A}} \Omega^{p} \mapsto \mathcal{A}^{\prime} \otimes_{\mathcal{A}} \Omega^{p+1}$, such that

$$
\nabla(\psi \lambda)=(\nabla \psi) \lambda+(-1)^{s} \psi d \lambda
$$

whenever $\psi \in \mathcal{A}^{\prime} \otimes_{\mathcal{A}} \Omega^{s}$ and $\lambda \in \Omega^{t}$. $\nabla$ is clearly not linear with respect to the algebra $\mathcal{A}$ but it is easy to check that the curvature $\nabla^{2}$ is a linear operator with respect to $\mathcal{A}$.

In the particular case where the module $\mathcal{A}^{\prime}$ is taken as the algebra $\mathcal{A}$ itself, any one-form $\omega$ (any element of $\Omega^{1}$ ) defines a covariant differential. One sets simply $\nabla \mathbb{1}=\omega$, where $\mathbb{1}$ is the unit of the algebra $\mathcal{A}$. When $f \in \mathcal{A}$, one obtains

$$
\nabla f=\nabla \mathbb{1} f=(\nabla \mathbb{1}) f+\mathbb{1} d f=d f+\omega f .
$$

Moreover, $\nabla^{2} f=\nabla(d f+\omega f)=d^{2} f+\omega d f+(\nabla \omega) f-\omega d f=(\nabla \omega) f$. The curvature, in that case, is

$$
\rho \doteq \nabla \omega=\nabla \mathbb{1} \omega=(\nabla \mathbb{1}) \omega+\mathbb{1} d \omega=d \omega+\omega^{2} .
$$

Take $u$ as an invertible element of $\mathcal{A}$, and act with $d$ on the equation $u^{-1} u=\mathbb{1}$. Using the property $d \mathbb{1}=0$ one obtains $d u^{-1}=-u^{-1} d u u^{-1}$. Define $\omega^{\prime}=u^{-1} \omega u+u^{-1} d u$ and compute the new curvature $\rho^{\prime}=d \omega^{\prime}+\omega^{\prime 2}$. One obtains immediately $\rho^{\prime}=u^{-1}\left(d \omega+\omega^{2}\right) u=u^{-1} \rho u$. This shows that the usual formulae hold without having to assume commutativity of the algebra $\mathcal{A}$.

Remark that here we take the module $\mathcal{A}^{\prime}$ (a necessary ingredient in the construction of any gauge theory) as the algebra $\mathcal{A}$ itself. More generally, we could have chosen a free module $\mathcal{A}^{p}$, or even a projective module over $\mathcal{A}$. We shall not consider here this more general situation. Therefore, in some sense, our connections are an analogue of usual "abelian Yang Mills fields", but the algebra $\mathcal{A}$, contrarily to what happens in conventional gauge field theories, will be non-commutative.

\subsection{Connections on $\mathcal{M}$ and their curvature}

We now return to the specific case where $\mathcal{A}=\mathcal{M}$ is the algebra of functions over the quantum plane at a cubic root of unity.

The most general connection is defined by an element $\phi$ of $\Omega_{W Z}^{1}(\mathcal{M})$. Since we have a quantum group action of $\mathcal{H}$ on $\Omega_{W Z}$, it is convenient to decompose $\phi$ into representations of this algebra as obtained in Section 7.5. We set

$$
\phi=\phi_{3 i}+\phi_{3 i}^{\prime}+\phi_{3 e}+\phi_{3 o}+\phi_{6 e},
$$

where

$$
\begin{aligned}
\phi_{3 i}= & a_{i 1}\left(q x^{2} y d x-d y\right)+a_{i 2}\left(q^{2} x y^{2} d x-x^{2} y d y\right)+a_{i 3} q\left(d x-x y^{2} d y\right) \\
\phi_{3 i}^{\prime}= & b_{i 1} q^{2} y d y+b_{i 2}(y d x+q x d y)+b_{i 3} q^{2} x d x \\
\phi_{3 e}= & a_{e 1} d y+a_{e 2}\left(q x y^{2} d x+x^{2} y d y\right)+a_{e 3} d x \\
\phi_{3 o}= & b_{o 1} q\left(x d x-x^{2} y^{2} d y\right)+b_{o 2} q(q y d x-x d y)+b_{o 3} q\left(x^{2} y^{2} d x-q y d y\right) \\
\phi_{6 e}= & c_{1}\left(x y d x-q^{2} x^{2} d y\right)+c_{2}\left(y^{2} d x-q x y d y\right)+c_{3} q x^{2} d x+c_{4} q y^{2} d y+ \\
& c_{5} q^{2}\left(x y d x+x^{2} d y\right)+c_{6} q\left(x y d y+y^{2} d x\right)
\end{aligned}
$$

The coefficients $a_{i}$ and $b_{i}$ refer to the three-dimensional irreducible representations, $a_{e}$ and $b_{o}$ to the even and odd three-dimensional indecomposable representations, and $c$ to the six-dimensional indecomposable representation $6_{\text {eve }}$.

The exact expression of the curvature $\rho=d \phi+\phi^{2}$ is not very illuminating, but, thanks to the knowledge of the general features of the multiplication in $\Omega_{W Z}$ (Section 7.8 ) and the properties of $d$ (Section 7.6) we can make the following observations: 
- $\phi \in 3_{\text {irr }} \subset 3_{\text {odd }} \otimes 2 \Rightarrow d \phi \in 3_{\text {irr }}, \phi^{2} \in 3_{\text {odd }}$. Actually, direct calculation shows that $\phi^{2}=0$, so $\rho=d \phi \in 3_{\text {irr }}$.

- $\phi \in 3_{\text {eve }} \subset 3_{\text {odd }} \otimes 2 \Rightarrow d \phi=0$, and $\rho=\phi^{2} \in 3_{\text {odd }}$.

- $\phi \in 3_{\text {irr }} \subset 3_{\text {eve }} \otimes 2 \Rightarrow d \phi=0$, and $\rho=\phi^{2} \in 3_{\text {irr }}$.

- $\phi \in 3_{\text {odd }} \subset 3_{\text {eve }} \otimes 2 \Rightarrow d \phi \in 3_{\text {odd }}, \phi^{2} \in 3_{\text {irr }}$. In fact, it can be shown that here also $\phi^{2}=0$, so $\rho=d \phi \in 3_{\text {odd }}$.

- $\phi \in 6_{\text {eve }}=3_{\text {irr }} \otimes 2 \Rightarrow d \phi \in 2, \phi^{2} \in 3_{\text {eve }}$, and $\rho \in 3_{\text {eve }}$.

\section{Hermitian connections}

As we know, the only star operation compatible with the quantum group action of $\mathcal{H}$ on the differential algebra $\Omega_{W Z}$, when $q^{3}=1$, is the one obtained in Section $7.7\left(d x^{*}=d x, d y^{*}=\right.$ $d y$ ). Imposing the hermiticity property $\phi=\phi^{*}$ on the connection implies that all the coefficients $a_{i}, b_{i}, a_{e}, b_{o}, c$ should be real.

Consider, for instance, the most general hermitian connection with coefficients in the representation $3_{\text {eve }}$, namely

$$
\phi=\phi_{3 e}=a_{e 1} d y+a_{e 3} d x+a_{e 2}\left(x^{2} y d y+q x y^{2} d x\right),
$$

with real coefficients $a_{e 1}, a_{e 2}, a_{e 3}$.

In this case, $d \phi=0$, automatically. The curvature is then equal to $\phi^{2}$ and its expression is quite simple, it reads

$$
\rho=\left(a_{e 1} a_{e 3}-a_{e 2}^{2}\right)(1-q) d x d y .
$$

Notice that it is a singlet, the one-dimensional representation obtained as a subrepresentation of the $3_{\text {odd }}$ of $\Omega_{W Z}^{2}$. 


\section{Incorporation of Space-Time}

\subsection{Algebras of differential forms over $C^{\infty}(M) \otimes \mathcal{M}$}

Let $\Lambda$ be the algebra of usual differential forms over a space-time manifold $M$ (the De Rham complex) and $\Omega_{W Z} \doteq \Omega_{W Z}(\mathcal{M})$, the differential algebra over the reduced quantum plane introduced in Section 7. Remember that $\Omega_{W Z}^{0}=\mathcal{M}, \Omega_{W Z}^{1}=\mathcal{M} d x+\mathcal{M} d y$, and that $\Omega_{W Z}^{2}=\mathcal{M} d x d y$. Calling $\Xi$ the graded tensor product of these two differential algebras,

$$
\Xi \doteq \Lambda \otimes \Omega_{W Z}
$$

we see that:

- An element of $\Xi^{0}=\Lambda^{0} \otimes \Omega_{W Z}^{0}$ is a $3 \times 3$ matrix with elements in $C^{\infty}(M)$. It can be thought as an $M_{3}(\mathbb{C})$-valued scalar field.

- A generic element of $\Xi^{1}=\left(\Lambda^{0} \otimes \Omega_{W Z}^{1}\right) \oplus\left(\Lambda^{1} \otimes \Omega_{W Z}^{0}\right)$ is given by a triplet $\omega=\left(A_{\mu}, \phi_{x}, \phi_{y}\right)$, where $A_{\mu}$ determines a one-form (a vector field) on the manifold $M$ with values in $M_{3}(\mathbb{C})$ (which can be considered as the Lie algebra of the Lie group $G L(3, \mathbb{C})$ ), and where $\phi_{x}$ and $\phi_{y}$ are $M_{3}(\mathbb{C})$-valued scalar fields. Indeed $\phi_{x}\left(x^{\mu}\right) d x+\phi_{y}\left(x^{\mu}\right) d y \in \Lambda^{0} \otimes \Omega_{W Z}^{1}$.

- An arbitrary element of $\Xi^{2}=\left(\Lambda^{0} \otimes \Omega_{W Z}^{2}\right) \oplus\left(\Lambda^{1} \otimes \Omega_{W Z}^{1}\right) \oplus\left(\Lambda^{2} \otimes \Omega_{W Z}^{0}\right)$ consists of

- $F_{\mu \nu} d x^{\mu} d x^{\nu} \in \Lambda^{2} \otimes \Omega_{W Z}^{0}$, a matrix-valued 2-form on the manifold $M$,

- a matrix-valued scalar field on $M$, i.e., an element of $\Lambda^{0} \otimes \Omega_{W Z}^{2}$,

- two matrix-valued vector fields on $M$, given by an element of $\Lambda^{1} \otimes \Omega_{W Z}^{1}$.

The algebra $\Xi$ is endowed with a differential (of square zero, of course, and obeying the Leibniz rule) defined by $d \doteq d \otimes i d \pm i d \otimes d$. Here \pm is the (differential) parity of the first factor of the tensor product upon which $d$ is applied, and the two $d$ 's appearing on the right hand side are the usual De Rham differential on antisymmetric tensor fields and the differential of the reduced Wess-Zumino complex, respectively.

If $G$ is a Lie group acting on the manifold $M$, it acts also (by pull-back) on the functions on $M$ and, more generally, on the differential algebra $\Lambda$. For instance, we may assume that $M$ is Minkowski space and $G$ is the Lorentz group. The Lie algebra of $G$ and its enveloping algebra $\mathcal{U}$ also act on $\Lambda$, by differential operators. Intuitively, elements of $\Xi$ have an "external" part (i.e., functions on $M$ ) on which $\mathcal{U}$ act, and an "internal" part (i.e., elements belonging to $\mathcal{M}$ ) on which $\mathcal{H}$ acts. We saw that $\mathcal{H}$ is a Hopf algebra (neither commutative nor cocommutative) whereas $\mathcal{U}$, as it is well known, is a non-commutative but cocommutative Hopf algebra. To conclude, we have an action of the Hopf algebra $\mathcal{U} \otimes \mathcal{H}$ on the differential algebra $\Xi$.

\subsection{Generalized gauge fields}

Since we have a differential algebra $\Xi$ on the associative algebra $C^{\infty}(M) \otimes \mathcal{M}$ we can define, as usual, "abelian"-like connections by choosing a module which is equal to the associative algebra itself. A Yang-Mills potential $\omega$ is an arbitrary element of $\Xi^{1}$ and the corresponding curvature, $d \omega+\omega^{2}$, is an element of $\Xi^{2}$. As we have said above, $\omega=\left(A_{\mu}, \phi_{x}, \phi_{y}\right)$ consists of a usual Yang-Mills field $A_{\mu}$ and a pair $\phi_{x}, \phi_{y}$ of scalar fields, all of them valued in $M_{3}(\mathbb{C})$. We have $\omega=A+\Phi$, where $A=A_{\mu} d x^{\mu}$ and $\Phi=\phi_{x} d x+\phi_{y} d y \in \Lambda^{0} \otimes \Omega_{W Z}^{1} \subset \Xi^{1}$. We can also decompose $A=A^{\alpha} \lambda_{\alpha}$, with $\lambda_{\alpha}$ denoting the usual Gell-Mann matrices (together with the unit matrix) and $A^{\alpha}$ a set of complex valued one-forms on the manifold $M$. To make the distinction clearer, let us call $\delta$ the differential on $\Xi, \underline{d}$ the differential on $\Lambda$ and $d$ the differential on $\Omega_{W Z}$ (as before). The curvature is then $\delta \omega+\omega^{2}$. Explicitly,

$$
\delta A=\left(\underline{d} A^{\alpha}\right) \lambda_{\alpha}-A^{\alpha} d \lambda_{\alpha}
$$

and

$$
\delta \Phi=\left(\underline{d} \phi_{x}\right) d x+\left(\underline{d} \phi_{y}\right) d y+\left(d \phi_{x}\right) d x+\left(d \phi_{y}\right) d y .
$$

It is therefore clear that the corresponding curvature will have several pieces: 
- The Yang-Mills strength $F$ of $A$,

$$
F \doteq\left(\underline{d} A^{\alpha}\right) \lambda_{\alpha}+A^{2} \quad \in \Lambda^{2} \otimes \Omega_{W Z}^{0}
$$

- A kinetic term $\mathcal{D} \Phi$ for the scalars, consisting of a purely derivative term, a covariant coupling to the gauge field and a mass term for the Yang-Mills field (linear in the $A_{\mu}$ 's),

$$
\mathcal{D} \Phi \doteq\left(\underline{d} \phi_{x}\right) d x+\left(\underline{d} \phi_{y}\right) d y+A \Phi+\Phi A-A^{\alpha} d \lambda_{\alpha} \in \Lambda^{1} \otimes \Omega_{W Z}^{1}
$$

- Finally, a self interaction term for the scalars

$$
\left(d \phi_{x}\right) d x+\left(d \phi_{y}\right) d y+\Phi^{2} \quad \in \Lambda^{0} \otimes \Omega_{W Z}^{2}
$$

We recover the usual ingredients of a Yang-Mills-Higgs system (the mass term for the gauge field, linear in $A$, is usually obtained from the " $A \Phi$ interaction" after shifting $\Phi$ by a constant).

By chosing an appropriate scalar product on the space $\Xi^{2}$, one obtains therefore a quantity that is quadratic in the curvatures (quartic in $A$ and $\Phi$ ) and could be a candidate for the Lagrangian of a theory of Yang-Mills-Higgs type. However, if we do not make specific choices for the connection (for instance by imposing reality constraints or by selecting one or another representation of $\mathcal{H}$ ), the results are a bit too general and, in any case, difficult to interpret physically. Regarding this construction, there is also another caveat that will be explained in the next section. 


\section{Discussion: quantum group symmetry and noncommut- ing fields}

As mentioned in Section 9.2, the building of physical models of gauge type will involve the consideration of one-forms. If we restrict ourselves to the "internal space" part of these one-forms, we have to consider objects of the form

$$
\Phi=\sum_{i} \varphi_{i} \omega_{i} .
$$

Here $\left\{\omega_{i}\right\}$ is a basis of some non-trivial indecomposable representation of $\mathcal{H}$ (or any other noncocommutative quantum group) on the space of 1 -forms, and $\varphi_{i}$ are functions over some space-time manifold. What about the transformation properties of the fields $\varphi_{i}$ ? This is a question of central importance, since, ultimately, we will integrate out the internal space (whatever this means), and the only relic of the quantum group action on the theory will be the transformations of the $\varphi_{i}$ 's. There are several possibilities: one of them, as suggested from the results of Section 9 is to consider $\mathcal{H}$ as a discrete analogue of the Lorentz group (actually, of the enveloping algebra $\mathcal{U}$ of its Lie algebra). In such a case, "geometrical quantities", like $\Phi$ should be $\mathcal{H}$-invariant (and $\mathcal{U}$-invariant). This requirement obviously forces the $\varphi_{i}$ to transform. Another possibility would be to assume that $\Phi$ itself transforms according to some representation of this quantum group (in the same spirit one can study, classically, the invariance of particularly chosen connections under the action of a group acting also on the base manifold). In any case, the $\varphi_{i}$ are going to span some non-trivial representation space of $\mathcal{H}$.

Usually, the components $\phi_{i}$ of fields are real (or complex) numbers and are, therefore, commuting quantities. However, this observation leads to the following problem: If the components of the fields commute, so that $\varphi_{i} \varphi_{j}=\varphi_{j} \varphi_{i}$, then we get $h .\left(\varphi_{i} \varphi_{j}\right)=h .\left(\varphi_{j} \varphi_{i}\right)$, for any $h \in \mathcal{H}$. This would imply (here $\Delta h=h_{1} \otimes h_{2}$ )

$$
\begin{aligned}
\left(h_{1} \cdot \varphi_{i}\right)\left(h_{2} \cdot \varphi_{j}\right) & =\left(h_{1} \cdot \varphi_{j}\right)\left(h_{2} \cdot \varphi_{i}\right) \\
& =\left(h_{2} \cdot \varphi_{i}\right)\left(h_{1} \cdot \varphi_{j}\right) .
\end{aligned}
$$

This equality cannot be true in general unless we have a cocommutative coproduct. Hence we should generally have a noncommutative product for the fields. In our specific case, there is only one abelian $\mathcal{H}$-module algebra, the $3_{\text {odd }}$ one (which is nothing else than the group algebra of the group $\mathbb{Z}_{3}$ ). Only fields transforming according to this representation could have an abelian product. However, covariance strongly restricts the allowable scalar products on each of the representation spaces (for instance, the results for the quantum group $\mathcal{H}$ are shown in Appendix E, where we get both indefinite and degenerate metrics). This fact is particularly important as one should have a positive definite metric on the physical degrees of freedom. To this end one should disregard the non-physical (gauge) ones, and look for representations such that only positive definite states survive. Thus we see that the selection of the representation space upon which to build the physical model is not easy.

The fact of having noncommuting fields has a certain resemblance with the case of supersymmetry. As the superspace algebra is noncommutative, the scalar superfield must have noncommutative component fields in order to match its transformation properties. In this last situation, everything can be casted in terms of Grassmann variables and fields. As a consequence, instead of having - on each space-time point - just the Grassmann algebra over the complex numbers, we see the appearance of an enlarged algebra generated by both the variables and fields. Introducing a $q$-deformed superspace leads to a more complicated algebraic structure [19]. Therefore, it is reasonable to expect that the addition of a non-trivial quantum group as a symmetry of the space forces an even more constrained algebra.

We should point out that the reasoning followed above is very general, and is independent of the details of the fields. That is, the arguing leading to such a conclusion relies only in the existence of a non-cocommutative Hopf algebra acting in a nontrivial way on the fields.

For this reason, we did not plan, in this paper, to make specific choices and discuss Lagrangian models. Actually, trying to write down such a definite physical model would involve the making 
of very particular choices; many of them are possible and we do not know, at the present level of our analysis, which kind of constraint could give rise to interesting physics.

Our purpose was rather to investigate the structures involved and work out (or present) the mathematical tools that would allow such physical applications. We hope that the present exposition of our recent work will trigger some interesting ideas in that direction. 


\section{Acknowledgements}

We are greatly indebted to Oleg Ogievetsky for his comments and discussions. 


\section{Appendices}

\section{Appendix A. Expression of Gell-Mann matrices in terms of $x$ and $y$}

Usual Gell-Mann matrices $\left\{\lambda_{i}\right\}_{i=1 \ldots 8}$ (a base for the Lie algebra of hermitian traceless $3 \times 3$ matrices) can be written in terms of elementary matrices, and therefore also in terms of the generators $x$ and $y$. Remember that

$$
\begin{array}{lll}
\lambda_{1}=E_{12}+E_{21} & \lambda_{2}=i\left(-E_{12}+E_{21}\right) & \lambda_{3}=E_{11}-E_{22} \\
\lambda_{4}=E_{13}+E_{31} & \lambda_{5}=i\left(-E_{13}+E_{31}\right) & \\
\lambda_{6}=E_{23}+E_{32} & \lambda_{7}=i\left(-E_{23}+E_{32}\right) & \lambda_{8}=\frac{1}{\sqrt{3}}\left(E_{11}+E_{22}-2 E_{33}\right)
\end{array}
$$

hence we obtain

$$
\begin{aligned}
& \lambda_{1}=\left(y+x y+y^{2}+x^{2} y+q x y^{2}+q^{2} x^{2} y^{2}\right) / 3 \\
& \lambda_{2}=-i\left(y+x y-y^{2}+x^{2} y-q x y^{2}-q^{2} x^{2} y^{2}\right) / 3 \\
& \lambda_{3}=\left((1-q) x+\left(1-q^{2}\right) x^{2}\right) / 3 \\
& \lambda_{4}=\left(y+q^{2} x y+y^{2}+q x^{2} y+x y^{2}+x^{2} y^{2}\right) / 3 \\
& \lambda_{5}=i\left(y+q^{2} x y-y^{2}+q x^{2} y-x y^{2}-x^{2} y^{2}\right) / 3 \\
& \lambda_{6}=\left(y+q x y+y^{2}+q^{2} x^{2} y+q^{2} x y^{2}+q x^{2} y^{2}\right) / 3 \\
& \lambda_{7}=-i\left(y+q x y-y^{2}+q^{2} x^{2} y-q^{2} x y^{2}-q x^{2} y^{2}\right) / 3 \\
& \lambda_{8}=-\left(q^{2} x+q x^{2}\right) / \sqrt{3}
\end{aligned}
$$

\section{Appendix B. A set of Gell-Mann matrices with coefficients in $\mathcal{F}$}

Here we replace $x$ and $y$ by $\Delta_{L} x$ and $\Delta_{L} y$ in the expression of the usual Gell-Mann matrices given in Appendix A. We obtain in this way a new set of matrices with entries in the quantum group $\mathcal{F}$, denoted by $\lambda_{i}^{\prime}$. We only show the results for $\lambda_{3}$ and $\lambda_{8}$ since we do not need these matrices explicitly in our work.

$$
\begin{aligned}
\lambda_{3}^{\prime} & \doteq \frac{1}{3}\left(\begin{array}{ccc}
(1-q) a+\left(1-q^{2}\right) a^{2} & (1-q) b+\left(q-q^{2}\right) a b & \left(1-q^{2}\right) b^{2} \\
\left(1-q^{2}\right) b^{2} & \left(q^{2}-1\right) a+(q-1) a^{2} & (1-q) b+(1-q) a b \\
(1-q) b+\left(q^{2}-1\right) a b & \left(1-q^{2}\right) b^{2} & \left(q-q^{2}\right) a+\left(q^{2}-q\right) a^{2}
\end{array}\right) \\
\lambda_{8}^{\prime} & \doteq \frac{1}{\sqrt{3}}\left(\begin{array}{ccc}
-q a-q a^{2} & -q^{2} b+a b & -q b^{2} \\
-q b^{2} & -a-q^{2} a^{2} & -q^{2} b+q^{2} a b \\
-q^{2} b+q a b & -q b^{2} & -q^{2} a-a^{2}
\end{array}\right)
\end{aligned}
$$

Using the commutation relations for the reduced quantum group $\mathcal{F}$, one can check that the usual commutation relations of $S U(3)$ are satisfied. One can also check that $\operatorname{Tr}\left(\lambda_{i}\right)=0$ and that $\operatorname{Tr}\left(\lambda_{i} \lambda_{j}\right)=2 \delta_{i j}\left(\right.$ when $i \neq j$ the trace turns out to be proportional to $\left.1+q+q^{2}=0\right)$.

\section{Appendix C. A faithful representation of $\mathcal{F}$}

Let $\xi_{1}$ and $\xi_{2}$ be two commuting symbols $\left(\xi_{1} \xi_{2}=\xi_{2} \xi_{1}\right)$ whose cube power vanishes $\left(\xi_{1}^{3}=\xi_{2}^{3}=0\right)$. Let us write

$$
b=\xi_{1}\left(\begin{array}{ccc}
0 & 1 & 0 \\
0 & 0 & 1 \\
1 & 0 & 0
\end{array}\right) \quad c=\xi_{2}\left(\begin{array}{ccc}
0 & 1 & 0 \\
0 & 0 & 1 \\
1 & 0 & 0
\end{array}\right) \quad d=\left(\begin{array}{ccc}
1 & 0 & 0 \\
0 & q^{-1} & 0 \\
0 & 0 & q^{-2}
\end{array}\right)
$$

and, given that $a \doteq(1+q b c) d^{2}$,

$$
a=\left(\begin{array}{ccc}
1 & 0 & \xi_{1} \xi_{2} \\
\xi_{1} \xi_{2} q & q & 0 \\
0 & \xi_{1} \xi_{2} q^{2} & q^{2}
\end{array}\right)
$$


It can be checked that this is a faithful representation of the algebra $\mathcal{F}$. It is a representation in terms of $3 \times 3$ matrices with entries in the ring generated over $\mathbb{C}$ by $1, \xi_{1}, \xi_{2}$. This representation is due to [9]. 


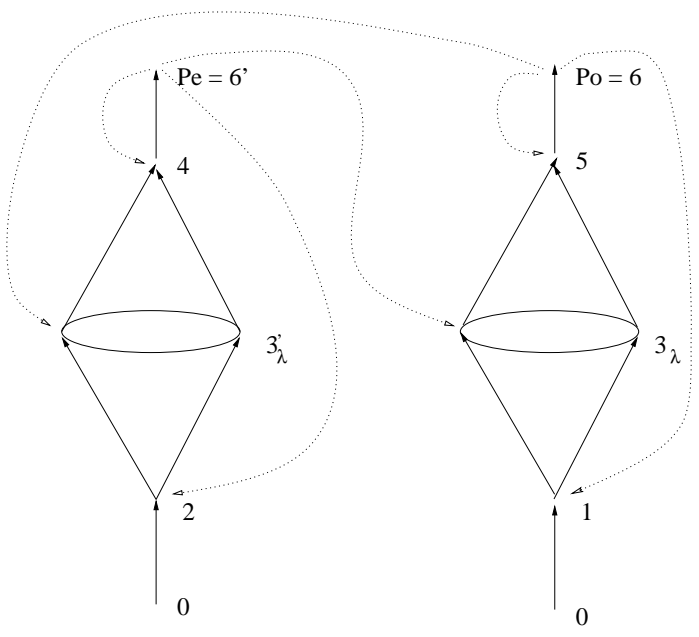

Figure 1: The lattices of submodules for the principal modules of $\mathcal{H}$.

\section{Appendix D. The lattice of submodules of $\mathcal{H}$}

The theory of complex representations of quantum groups of type $U_{q}(s l(2, \mathbb{C}))$ at roots of unity has been investigated by a number of people, but it is not yet in a satisfactory state. Here we are interested in representation theory of the finite-dimensional quotient $\mathcal{H}$. Notice that these representations can be considered as particular representations $\rho$ of $U_{q}(\operatorname{sl}(2, \mathbb{C}))$, namely those for which $\rho\left(X_{+}^{3}\right)=\rho\left(X_{-}^{3}\right)=0$ and $\rho\left(K^{3}\right)=1$. The structure of the regular representation was studied in [3] and the representation theory - in particular the lattice of submodules - was given in [4]; the general study, for arbitrary $N$, is to be found in [9]. For the reduced algebra $\mathcal{H}$ there are three principal modules (i.e., projective indecomposable modules). One is three-dimensional and irreducible. The two others, $6_{\text {eve }}$ and $6_{\text {odd }}$ are six-dimensional. When $\mathcal{H}$ is written in terms of matrices with entries in the Grassmann algebra with two generators, these two representations can be written as $6_{\text {odd }} \doteq\left(\gamma \theta_{1}+\delta \theta_{2}, \gamma^{\prime} \theta_{1}+\delta^{\prime} \theta_{2}, \alpha+\beta \theta_{1} \theta_{2}\right)$ and $6_{\text {eve }} \doteq\left(\alpha+\beta \theta_{1} \theta_{2}, \alpha^{\prime}+\beta^{\prime} \theta_{1} \theta_{2}, \gamma \theta_{1}+\delta \theta_{2}\right)$. Their lattices of submodules are obtained by requiring stability under the $\mathcal{H}$ action. They are given in Figure 1. The notation $P_{o} \equiv 6_{\text {odd }}$ and $P_{e} \equiv 6_{\text {eve }}$ refers to the fact that, when quotiented out by their respective radicals, those two indecomposable modules give irreducible representations of odd and even dimensions.

Remember that there exists a one-to-one correspondence between irreducible representations of the algebra $\mathcal{H}$ and the principal modules $3_{\text {irr }}, 6_{\text {eve }}$ and $6_{\text {odd }}$. Irreducible representations are obtained from these principal modules by factorizing their radical, which amounts to kill the Grassmann " $\theta$ " variables. As seen on the figure, the radical of $6_{\text {eve }}$ is of dimension 4 and the radical of $6_{\text {odd }}$, of dimension 5 . This gives us three irreducible representations, of dimensions 3 , $2=6-4$ and $1=6-5$. These are the three irreducible representations corresponding to the quotient $\overline{\mathcal{H}}$ of $\mathcal{H}$ by its Jacobson radical: namely $\overline{\mathcal{H}}=\mathbb{C} \oplus M_{2}(\mathbb{C}) \oplus M_{3}(\mathbb{C})$. The dashed lines in Figure 1 refer to the projective covers of the various representations.

The explicit definition given for $\mathcal{H}$ allows one to compute any tensor product of representations and reduce them. Here we consider only the tensor products of projective indecomposable representations $\left(\underline{6}_{o d d}, \underline{6}_{\text {eve }}\right.$ and $\left.\underline{3}_{i r r}\right)$ and the nontrivial irreducible ones $\left(\underline{2}\right.$ and $\left.\underline{3}_{i r r}\right)$.

$$
\begin{aligned}
& \underline{2} \times \underline{2} \quad \equiv \underline{1}^{+} \underline{3}_{i r r} \quad \underline{6}_{\text {eve }} \times \underline{3}_{i r r} \equiv 2\left(\underline{6}_{\text {eve }}\right)+2\left(\underline{3}_{i r r}\right) \\
& \underline{2} \times \underline{3}_{i r r} \equiv \underline{6}_{\text {eve }} \underline{3}_{\text {irr }} \quad \underline{6}_{\text {odd }} \times \underline{3}_{i r r} \equiv 2\left(\underline{6}_{\text {eve }}\right)+2\left(\underline{3}_{i r r}\right) \\
& \underline{3}_{i r r} \times \underline{3}_{i r r} \equiv \underline{6}_{\text {odd }}+\underline{3}_{i r r} \quad \underline{6}_{\text {eve }} \times \underline{6}_{\text {eve }} \equiv 2\left(\underline{6}_{\text {eve }}\right)+2\left(\underline{6}_{\text {odd }}\right)+4\left(\underline{3}_{\text {irr }}\right) \\
& \underline{6}_{\text {eve }} \times \underline{2}^{\prime} \equiv \underline{6}_{\text {odd }}+2\left(\underline{3}_{\text {irr }}\right) \quad \underline{6}_{\text {eve }} \times \underline{6}_{\text {odd }} \equiv 2\left(\underline{6}_{\text {eve }}\right)+2\left(\underline{6}_{\text {odd }}\right)+4\left(\underline{3}_{\text {irr }}\right) \\
& \left.\underline{6}_{o d d} \times \underline{2} \equiv \underline{6}_{\text {eve }}+2 \underline{3}_{i r r}\right) \quad \underline{6}_{o d d} \times \underline{6}_{o d d} \equiv 2\left(\underline{6}_{\text {eve }}\right)+2\left(\underline{6}_{\text {odd }}\right)+4\left(\underline{3}_{i r r}\right)
\end{aligned}
$$




\section{Appendix E. Metrics on indecomposable representations of $\mathcal{H}$}

Using the unique Hopf compatible star operation $*$ on $\mathcal{H}$, we can calculate the most general metric on the vector spaces of each of the indecomposable representations of $\mathcal{H}$. Obviously, as we did in Section 5.5, we restrict the inner product to be a quantum group invariant one.

On each representation space we use the basis obtained from appropriate restrictions of the natural basis of the column representations of $\mathcal{H}$ given in Section 4.5. For each indecomposable representation, we first write down the matrices of $X_{+}, X_{-}$and $K$ in the selected base, then we give an explicit expression of the most general covariant metric, and finally we calculate its signature.

$\bullet \mathbf{3}_{i r r}$

$$
X_{+}=\left(\begin{array}{ccc}
0 & 1 & 0 \\
0 & 0 & 1 \\
0 & 0 & 0
\end{array}\right) \quad X_{-}=\left(\begin{array}{ccc}
0 & 0 & 0 \\
-1 & 0 & 0 \\
0 & -1 & 0
\end{array}\right) \quad K=\left(\begin{array}{ccc}
q^{2} & 0 & 0 \\
0 & 1 & 0 \\
0 & 0 & q
\end{array}\right)
$$

We get for the metric, up to a real global normalization,

$$
G=\left(\begin{array}{ccc}
0 & 0 & -q^{2} \\
0 & 1 & 0 \\
-q & 0 & 0
\end{array}\right)
$$

Diagonalizing this matrix we get $G \sim \operatorname{Diag}(1,1,-1)$, so the signature is

$$
\sigma=(++-) \text {. }
$$

- $\mathbf{6}_{\text {odd }}$

$$
\begin{gathered}
X_{+}=\left(\begin{array}{cccccc}
0 & 0 & 1 & 0 & 0 & 0 \\
0 & 0 & 0 & 1 & 0 & 0 \\
0 & 0 & 0 & 0 & 1 & 0 \\
0 & 0 & 0 & 0 & 0 & 0 \\
0 & 0 & 0 & 0 & 0 & 0 \\
0 & 1 & 0 & 0 & 0 & 0
\end{array}\right) \quad X_{-}=\left(\begin{array}{cccccc}
0 & 0 & 0 & 0 & 0 & 0 \\
0 & 0 & 0 & 0 & 1 & 0 \\
1 & 0 & 0 & 0 & 0 & 0 \\
0 & 1 & 0 & 0 & 0 & 0 \\
0 & 0 & 0 & 0 & 0 & 0 \\
0 & 0 & 1 & 0 & 0 & 0
\end{array}\right) \\
K=\left(\begin{array}{cccccc}
q & 0 & 0 & 0 & 0 & 0 \\
0 & q & 0 & 0 & 0 & 0 \\
0 & 0 & q^{2} & 0 & 0 & 0 \\
0 & 0 & 0 & q^{2} & 0 & 0 \\
0 & 0 & 0 & 0 & 1 & 0 \\
0 & 0 & 0 & 0 & 0 & 1
\end{array}\right)
\end{gathered}
$$

Up to a normalization, the metric should be $(\beta \in \mathbb{R})$

$$
G=\left(\begin{array}{cccccc}
0 & 0 & 0 & q & 0 & 0 \\
0 & 0 & -q & 0 & 0 & 0 \\
0 & -q^{2} & 0 & 0 & 0 & 0 \\
q^{2} & 0 & 0 & 0 & 0 & 0 \\
0 & 0 & 0 & 0 & \beta & 1 \\
0 & 0 & 0 & 0 & 1 & 0
\end{array}\right) .
$$

A change of basis tells us that $G \sim \operatorname{Diag}\left(1,1,-1,-1, \lambda_{+}, \lambda_{-}\right)$, with $\lambda_{+}>0, \lambda_{-}<0$. Thus, in this case, the signature is

$$
\sigma=(+++---) \text {. }
$$


- $\boldsymbol{5}_{\text {odd }}$

$$
X_{+}=\left(\begin{array}{ccccc}
0 & 0 & 1 & 0 & 0 \\
0 & 0 & 0 & 1 & 0 \\
0 & 0 & 0 & 0 & 0 \\
0 & 0 & 0 & 0 & 0 \\
0 & 1 & 0 & 0 & 0
\end{array}\right) \quad X_{-}=\left(\begin{array}{ccccc}
0 & 0 & 0 & 0 & 0 \\
0 & 0 & 0 & 0 & 0 \\
1 & 0 & 0 & 0 & 0 \\
0 & 1 & 0 & 0 & 0 \\
0 & 0 & 1 & 0 & 0
\end{array}\right) \quad K=\left(\begin{array}{ccccc}
q & 0 & 0 & 0 & 0 \\
0 & q & 0 & 0 & 0 \\
0 & 0 & q^{2} & 0 & 0 \\
0 & 0 & 0 & q^{2} & 0 \\
0 & 0 & 0 & 0 & 1
\end{array}\right)
$$

Up to a real factor, the metric we obtain is $(\beta, \gamma \in \mathbb{R}, g \in \mathbb{C})$

$$
G=\left(\begin{array}{ccccc}
0 & 0 & i q \gamma & g & 0 \\
0 & 0 & -q^{2} \bar{g} & i q \beta & 0 \\
-i q^{2} \gamma & -q g & 0 & 0 & 0 \\
\bar{g} & -i q^{2} \beta & 0 & 0 & 0 \\
0 & 0 & 0 & 0 & 0
\end{array}\right)
$$

Its signature is

$$
\sigma=(++--0),
$$

because $G \sim \operatorname{Diag}\left(\lambda_{+},-\lambda_{+}, \lambda_{-},-\lambda_{-}, 0\right)$.

- $\mathbf{3}_{\text {odd }}$

$$
X_{+}=\left(\begin{array}{ccc}
0 & 1 & 0 \\
0 & 0 & 0 \\
\lambda_{2} & 0 & 0
\end{array}\right) \quad X_{-}=\left(\begin{array}{ccc}
0 & 0 & 0 \\
1 & 0 & 0 \\
0 & \lambda_{1} & 0
\end{array}\right) \quad K=\left(\begin{array}{ccc}
q & 0 & 0 \\
0 & q^{2} & 0 \\
0 & 0 & 1
\end{array}\right)
$$

Up to a real factor,

$$
G=\left(\begin{array}{ccc}
0 & i q & 0 \\
-i q^{2} & 0 & 0 \\
0 & 0 & 0
\end{array}\right)
$$

thus

$$
\sigma=(+-0)
$$

- $\mathbf{6}_{\text {eve }}$

$$
\begin{gathered}
X_{+}=\left(\begin{array}{cccccc}
0 & 0 & 1 & 0 & 0 & 0 \\
0 & 0 & -1 / 2 & 1 & 0 & 0 \\
0 & 0 & 0 & 0 & 0 & 0 \\
0 & 0 & 0 & 0 & 0 & 1 \\
1 & 0 & 0 & 0 & 0 & 0 \\
0 & 0 & 0 & 0 & 0 & 0
\end{array}\right) \quad X_{-}=\left(\begin{array}{ccccccc}
0 & 0 & 0 & 0 & 0 & 0 \\
0 & 0 & 0 & 0 & -1 & 0 \\
1 & 0 & 0 & 0 & 0 & 0 \\
-1 / 2 & 1 & 0 & 0 & 0 & 0 \\
0 & 0 & 0 & 0 & 0 & 0 \\
0 & 0 & -1 & 0 & 0 & 0
\end{array}\right) \\
K=\left(\begin{array}{cccccc}
q & 0 & 0 & 0 & 0 & 0 \\
0 & q & 0 & 0 & 0 & 0 \\
0 & 0 & q^{2} & 0 & 0 & 0 \\
0 & 0 & 0 & q^{2} & 0 & 0 \\
0 & 0 & 0 & 0 & 1 & 0 \\
0 & 0 & 0 & 0 & 0 & 1
\end{array}\right)
\end{gathered}
$$

Up to a normalization, the metric should be $(\beta \in \mathbb{R})$

$$
G=\left(\begin{array}{cccccc}
0 & 0 & i q \beta & -i q & 0 & 0 \\
0 & 0 & -i q & 0 & 0 & 0 \\
-i q^{2} \beta & i q^{2} & 0 & 0 & 0 & 0 \\
i q^{2} & 0 & 0 & 0 & 0 & 0 \\
0 & 0 & 0 & 0 & 0 & i \\
0 & 0 & 0 & 0 & -i & 0
\end{array}\right)
$$

The signature is

$$
\sigma=(+++---)
$$

because diagonalizing $G$ we find $G \sim \operatorname{Diag}\left(1,-1, \lambda_{+},-\lambda_{+}, \lambda_{-},-\lambda_{-}\right)$with $\lambda_{+}>0, \lambda_{-}<0$. 
- $4_{\text {eve }}$

$$
X_{+}=\left(\begin{array}{cccc}
0 & 1 & 0 & 0 \\
0 & 0 & 0 & 1 \\
0 & 0 & 0 & 0 \\
0 & 0 & 0 & 0
\end{array}\right) \quad X_{-}=\left(\begin{array}{cccc}
0 & 0 & -1 & 0 \\
1 & 0 & 0 & 0 \\
0 & 0 & 0 & 0 \\
0 & 0 & 0 & 0
\end{array}\right) \quad K=\left(\begin{array}{cccc}
q & 0 & 0 & 0 \\
0 & q^{2} & 0 & 0 \\
0 & 0 & 1 & 0 \\
0 & 0 & 0 & 1
\end{array}\right)
$$

We obtain the metric $(\alpha, \beta \in \mathbb{R}, g \in \mathbb{C})$

$$
G=\left(\begin{array}{cccc}
0 & 0 & 0 & 0 \\
0 & 0 & 0 & 0 \\
0 & 0 & \alpha & g \\
0 & 0 & \bar{g} & \beta
\end{array}\right)
$$

The signature is now dependant upon the parameters, because the non-null block is an arbitrary hermitian matrix.

$\bullet \mathbf{3}_{\text {eve }}$

$$
X_{+}=\left(\begin{array}{ccc}
0 & 1 & 0 \\
0 & 0 & \lambda_{2} \\
0 & 0 & 0
\end{array}\right) \quad X_{-}=\left(\begin{array}{ccc}
0 & 0 & -\lambda_{1} \\
1 & 0 & 0 \\
0 & 0 & 0
\end{array}\right) \quad K=\left(\begin{array}{ccc}
q & 0 & 0 \\
0 & q^{2} & 0 \\
0 & 0 & 1
\end{array}\right)
$$

In this case, we find simply

$$
G=\left(\begin{array}{lll}
0 & 0 & 0 \\
0 & 0 & 0 \\
0 & 0 & 1
\end{array}\right)
$$

- $2_{\text {eve }}$

$$
X_{+}=\left(\begin{array}{cc}
0 & 1 \\
0 & 0
\end{array}\right) \quad X_{-}=\left(\begin{array}{cc}
0 & 0 \\
1 & 0
\end{array}\right) \quad K=\left(\begin{array}{cc}
q & 0 \\
0 & q^{2}
\end{array}\right)
$$

Therefore

$$
G=\left(\begin{array}{cc}
0 & i q \\
-i q^{2} & 0
\end{array}\right) \sim \operatorname{Diag}(1,-1)
$$




\section{Appendix F. The space of differential operators on $\mathcal{M}$}

We now look at the structure of differential operators on the reduced quantum plane $\mathcal{M}$, i.e., the algebra of $3 \times 3$ matrices.

The operators $\partial_{x}$ and $\partial_{y}$

We already know what the operator $d: \Omega_{W Z}^{0}=\mathcal{M} \longrightarrow \Omega_{W Z}^{1}$ is.

A priori we can set $d f=d x \partial_{x}(f)+d y \partial_{y}(f)$, where $\partial_{x}(f), \partial_{y}(f) \in \mathcal{M}$. This equation defines $\partial_{x}$ and $\partial_{y}$ as (linear) operators on $\mathcal{M}$. We shall see later that they are twisted derivations on the algebra $\mathcal{M}$. This definition implies in particular (take $f=x$ or $f=y$ ):

$$
\begin{array}{ll}
\partial_{x}(x)=1 & \partial_{y}(x)=0 \\
\partial_{x}(y)=0 & \partial_{y}(y)=1
\end{array}
$$

\section{The space $\mathcal{D}$ of differential operators on $\mathcal{M}$}

Generally speaking, operators of the type $f(x, y) \partial_{x}$ or $f(x, y) \partial_{y}$ are called differential operators of order 1. Composition of such operators gives rise to differential operators of order higher than 1. Multiplication by an element of $\mathcal{M}$ is considered as a differential operator of degree 0 . The space of all these operators is a vector space $\mathcal{D}=\oplus_{i=0}^{4} \mathcal{D}_{i}$, graded by operators of

Order 0: $x^{r} y^{s} \cdot \operatorname{dim}\left(\mathcal{D}_{0}\right)=9$.

Order 1: $x^{r} y^{s} \partial_{x}, x^{r} y^{s} \partial_{y} \cdot \operatorname{dim}\left(\mathcal{D}_{1}\right)=9+9=18$.

Order 2: $x^{r} y^{s} \partial_{x} \partial_{x}, x^{r} y^{s} \partial_{x} \partial_{y}, x^{r} y^{s} \partial_{y} \partial_{y} \cdot \operatorname{dim}\left(\mathcal{D}_{2}\right)=9+9+9=27$.

Order 3: $x^{r} y^{s} \partial_{x} \partial_{x} \partial_{y}, x^{r} y^{s} \partial_{x} \partial_{y} \partial_{y} \cdot \operatorname{dim}\left(\mathcal{D}_{3}\right)=9+9=18$.

Order 4: $x^{r} y^{s} \partial_{x} \partial_{x} \partial_{y} \partial_{y} \cdot \operatorname{dim}\left(\mathcal{D}_{4}\right)=9$

All these operators are linearly independent. Moreover, since $\operatorname{dim}(\mathcal{D})=9+18+27+18+9=$ $81=9^{2}=\operatorname{dim}(\operatorname{End}(\mathcal{M}))$, we can identify $\mathcal{D}$ with $\operatorname{End}(\mathcal{M})$.

Warning: We have here a problem of notations: the reader should distinguish, for instance, $\partial_{x} f$ which is a differential operator from $\mathcal{M}$ to $\mathcal{M}$ (namely, when acting on something, it multiplies what follows by $f$, and then acts with $\partial_{x}$ on the result) from $\partial_{x}(f)$ which is the evaluation of $\partial_{x}$ on $f$, hence an element of $\mathcal{M}$ (which can, in turn, be considered as a differential operator of order $0)$. The presence, or absence, of parenthesis should be enough to make this clear.

\section{The twisting automorphisms $\sigma$ and $\tau$ ?}

Since we know how to commute $x, y$ with $d x, d y$, we can write, for any element $f \in \mathcal{M}$

$$
\begin{aligned}
& f d x=d x \sigma_{x}^{x}(f)+d y \sigma_{y}^{x}(f) \\
& f d y=d x \sigma_{x}^{y}(f)+d y \sigma_{y}^{y}(f)
\end{aligned}
$$

where each matrix element of

$$
\left(\begin{array}{cc}
\sigma_{x}^{x} & \sigma_{x}^{y} \\
\sigma_{y}^{x} & \sigma_{y}^{y}
\end{array}\right)
$$

is an element of $\operatorname{End}(\mathcal{M})$ to be determined. In particular, taking $f=x$ and $f=y$ in the above equations leads to

$$
\begin{aligned}
\sigma_{x}^{x}(x)=q^{2} x & \sigma_{y}^{x}(x)=0 \\
\sigma_{x}^{x}(y)=q y & \sigma_{y}^{x}(y)=0 \\
\sigma_{x}^{y}(x)=\left(q^{2}-1\right) y & \sigma_{y}^{y}(x)=q x \\
\sigma_{x}^{y}(y)=0 & \sigma_{y}^{y}(y)=q^{2} y
\end{aligned}
$$

${ }^{9}$ Some properties of these automorphisms are discussed in 20 
Moreover, let $f$ and $g$ be elements of $\mathcal{M}$. Using the associativity property $(f g) d x=f(g d x)$, and the same with $d y$, we find

$$
\sigma_{i}^{j}(f g)=\sigma_{i}^{k}(f) \sigma_{k}^{j}(g),
$$

with a summation over the repeated index $k \in\{x, y\}$. Thus the map $\sigma: f \in \mathcal{M} \rightarrow \sigma(f) \in M_{2}(\mathcal{M})$ is an algebra homomorphism $(\sigma(f g)=\sigma(f) \sigma(g))$ from $\mathcal{M}$ to the algebra $M_{2}(\mathcal{M})$ of $2 \times 2$ matrices with elements in $\mathcal{M}$.

In the same way, we could have written for any element $f \in \mathcal{M}$ (note the transposed index convention)

$$
\begin{aligned}
& d x f=\tau_{x}^{x}(f) d x+\tau_{x}^{y}(f) d y \\
& d y f=\tau_{y}^{x}(f) d x+\tau_{y}^{y}(f) d y
\end{aligned}
$$

hence defining another $\mathcal{M} \rightarrow M_{2}(\mathcal{M})$ homomorphism $\tau$, since $\tau_{i}^{j}(f g)=\tau_{i}^{k}(f) \tau_{k}^{j}(g)$.

\section{$\partial_{x}$ and $\partial_{y}$ are twisted derivations}

The usual Leibniz rule for $d$, namely $d(f g)=d(f) g+f d(g)$, implies

$$
\left(\begin{array}{c}
\partial_{x}(f g) \\
\partial_{y}(f g)
\end{array}\right)=\left(\begin{array}{c}
\partial_{x}(f) g \\
\partial_{y}(f) g
\end{array}\right)+\left(\begin{array}{cc}
\sigma_{x}^{x}(f) & \sigma_{x}^{y}(f) \\
\sigma_{y}^{x}(f) & \sigma_{y}^{y}(f)
\end{array}\right)\left(\begin{array}{c}
\partial_{x}(g) \\
\partial_{y}(g)
\end{array}\right)
$$

or even

$$
\partial_{i}(f g)=\partial_{i}(f) g+\sigma_{i}^{j}(f) \partial_{j}(g) .
$$

This shows that $\partial_{x}$ and $\partial_{y}$ are twisted derivations (derivations twisted by an homomorphism). Actually, it is conceptually interesting to notice that one can get rid of the (explicit) twisting by introducing two distinct module structures on $\Omega_{W Z}^{1}$. First of all, elements of $\Omega_{W Z}^{1}$ are of the kind $d x g_{1}+d y g_{2}$ and can be written as a column vector $\left(\begin{array}{l}g_{1} \\ g_{2}\end{array}\right)$. We can then consider $\Omega_{W Z}^{1} \simeq \mathcal{M}^{2}$ as an $\mathcal{M}$-bimodule, taking as right module structure the standard one:

$$
\left(\begin{array}{c}
g_{1} \\
g_{2}
\end{array}\right) \cdot f \doteq\left(\begin{array}{c}
g_{1} f \\
g_{2} f
\end{array}\right)
$$

and making use of the automorphism $\sigma$ for the left module structure:

$$
f \cdot\left(\begin{array}{l}
g_{1} \\
g_{2}
\end{array}\right) \doteq\left(\begin{array}{cc}
\sigma_{x}^{x}(f) & \sigma_{x}^{y}(f) \\
\sigma_{y}^{x}(f) & \sigma_{y}^{y}(f)
\end{array}\right)\left(\begin{array}{l}
g_{1} \\
g_{2}
\end{array}\right) .
$$

The operator $\underline{d} \doteq\left(\begin{array}{c}\partial_{x} \\ \partial_{y}\end{array}\right)$ is a derivation on the algebra $\mathcal{M}$ with values in the bimodule $\Omega_{W Z}^{1} \simeq \mathcal{M}^{2}$. Indeed, by using the two (distinct) left and right module structures just given, the twisted derivation properties of the operators $\partial_{x}$ and $\partial_{y}$, when expressed in terms of $\underline{d}$, read simply

$$
\underline{d}(f g)=\underline{d}(f) \cdot g+f \cdot \underline{d}(g) .
$$

\section{Relations in $\mathcal{D}$}

For calculational purposes, it is useful to know the commutation relations between $x, y$ and $\partial_{x}, \partial_{y}$, those between $\partial_{x}$ and $\partial_{y}$, and the relations between the $\sigma_{i}^{j}$. Calculations are straightforward but slightly cumbersome... here are the results (see also [6, 8]).

Calculating $\partial_{x}(x f), \partial_{x}(y f), \partial_{y}(x f), \partial_{y}(y f)$ gives the following relations (here we do not suppose that $q^{N}=1$ ):

$$
\begin{aligned}
& \partial_{x} x=1+q^{2} x \partial_{x}+\left(q^{2}-1\right) y \partial_{y} \\
& \partial_{x} y=q y \partial_{x} \\
& \partial_{y} x=q x \partial_{y}
\end{aligned}
$$




$$
\partial_{y} y=1+q^{2} y \partial_{y}
$$

One can check that all these relations are compatible with the defining relations of $\mathcal{M}$.

We then calculate $\partial_{x} \partial_{y}(x), \partial_{x} \partial_{y}(y), \partial_{y} \partial_{x}(x), \partial_{y} \partial_{x}(y)$. Compatibility of the results implies the commutation relation

$$
\partial_{y} \partial_{x}=q \partial_{x} \partial_{y}
$$

Moreover, the fact that $\partial_{x, y}^{3}$ are central elements of $\mathcal{D}$ if $q^{3}=1$ leads to

$$
\partial_{x}^{3}=\partial_{y}^{3}=0 \text {. }
$$

The commutation relations between the $\sigma$ 's can be obtained from the values of the $\sigma_{j}^{i}(x)$. Taking into account that $\sigma_{y}^{x} \equiv 0$, the remaining non-trivial relations are

$$
\begin{aligned}
\sigma_{x}^{x} \sigma_{x}^{y} & =q^{2} \sigma_{x}^{y} \sigma_{x}^{x} \\
\sigma_{x}^{x} \sigma_{y}^{y} & =\sigma_{y}^{y} \sigma_{x}^{x} \\
\sigma_{x}^{y} \sigma_{y}^{y} & =q^{2} \sigma_{y}^{y} \sigma_{x}^{y}
\end{aligned}
$$

\section{Scaling operators}

It is useful to introduce the operators [21]

$$
\begin{aligned}
& \mu_{x}=1+\left(q^{2}-1\right)\left(x \partial_{x}+y \partial_{y}\right) \\
& \mu_{y}=1+\left(q^{2}-1\right) y \partial_{y}
\end{aligned}
$$

Indeed,

$$
\begin{array}{ll}
\mu_{x} x=q^{2} x \mu_{x} & \mu_{y} x=x \mu_{y} \\
\mu_{x} y=q^{2} y \mu_{x} & \mu_{y} y=q^{2} y \mu_{y}
\end{array}
$$

Observe that $\mu_{x}$ rescales $x$ and $y$ in the same way. This is not so for $\mu_{y}$. Their product satisfies

$$
\mu_{x} \mu_{y}=\mu_{y} \mu_{x}
$$

and also

$$
\mu_{x}^{3}=\mu_{y}^{3}=\mathbb{1} .
$$

It is sometimes handy to rewrite the commutation relations between $x, y$ and the first order operators $\partial_{x}, \partial_{y}$ in terms of the $\mu_{x}, \mu_{y}$. For instance,

$$
\begin{aligned}
& \partial_{x} x=\mu_{x}+x \partial_{x} \\
& \partial_{y} y=\mu_{y}+y \partial_{y}
\end{aligned}
$$

\section{The action of $\mathcal{H}$ in terms of differential operators}

The twisted derivations $\partial_{x}, \partial_{y}$ considered previously constitute a $q$-analogue of the notion of vector fields. Their powers (including zero) build up arbitrary differential operators. Elements of $\mathcal{H}$ act also like powers of generalized vector fields (consider for instance the left action generated by $\left.X_{ \pm}^{L}, K^{L}\right)$, but, of course, they are differential operators of a special kind: remember that $\operatorname{dim}(\mathcal{H})=27$ whereas $\operatorname{dim}(\mathcal{D})=81$. One can say that elements of $\mathcal{H}$ act on $\mathcal{M}$ as fundamental differential operators since they are associated with the action of a (quantum) group on a (quantum) space.

A priori, the generators $X_{ \pm}^{L}, K^{L}$ can be written in terms of $x, y, \partial_{x}, \partial_{y}$. In order to find these expressions, it helps to notice that $\partial_{x}$ and $\partial_{y}$ are respectively of weight $-1 / 2,1 / 2$ in $\mathbb{Z} / 3 \mathbb{Z}$ (see also the discussion at the end of Section 4.4). Writing the generators as arbitrary differential operators 
of a fixed weight, one can determine the coefficients by imposing that equations (14), (22) are satisfied. A rather cumbersome calculation leads to the following unique solution:

$$
\begin{aligned}
X_{+}^{L} & =x \partial_{y}+(q-1) x y \partial_{y}^{2} \\
X_{-}^{L} & =y \partial_{x}+\left(q-q^{2}\right) x y \partial_{x}^{2}+(1-q) y^{2} \partial_{x} \partial_{y} \\
K^{L} & =\mathbb{1}+(q-1) x \partial_{x}+\left(q^{2}-1\right) y \partial_{y}-3 q x^{2} \partial_{x}^{2}+3(1-q) x^{2} y \partial_{x}^{2} \partial_{y}+9 x^{2} y^{2} \partial_{x}^{2} \partial_{y}^{2} \\
K_{-}^{L} & \equiv\left(K^{L}\right)^{2}=\mathbb{1}+\left(q^{2}-1\right) x \partial_{x}+(q-1) y \partial_{y}-3 x y \partial_{x} \partial_{y}-3 q y^{2} \partial_{y}^{2}
\end{aligned}
$$

An alternative way of writing these, is to make use of the scaling operators,

$$
\begin{aligned}
K_{-}^{L} & =\mu_{x} \mu_{y} \\
K^{L} & =\mu_{x}^{2} \mu_{y}^{2} \\
X_{+}^{L} & =\mu_{y}^{2} x \partial_{y} \\
X_{-}^{L} & =q \mu_{x} y \partial_{x}
\end{aligned}
$$




\section{Appendix G. The universal $R$ matrix of $\mathcal{H}$}

We did not discuss the $R$-matrix aspects of $\mathcal{H}$ in the main body of this paper, however it can be seen that $\mathcal{H}$ is actually a braided and quasi-triangular finite-dimensional Hopf algebra - as it is well known, the quantum enveloping algebra of $S L(2)$ does not posess these properties when $q$ is a root of unity. The universal $R$ matrix can be obtained directly from a general formula given in 22. but one also obtain in a pedestrian way. In any case, the answer is simply the following:

$$
\begin{aligned}
R= & \frac{1}{3 q}\left[\mathbb{1} \otimes \mathbb{1}+(\mathbb{1} \otimes K+K \otimes \mathbb{1})+\left(\mathbb{1} \otimes K^{2}+K^{2} \otimes \mathbb{1}\right)\right. \\
& \left.+q^{2}\left(K \otimes K^{2}+K^{2} \otimes K\right)+q K \otimes K+q K^{2} \otimes K^{2}\right] \\
& \times\left[\mathbb{1} \otimes \mathbb{1}+\left(q-q^{-1}\right) X_{-} \otimes X_{+}+3 q X_{-}^{2} \otimes X_{+}^{2}\right]
\end{aligned}
$$

By using the explicit formulae for the generators $X_{+}, X_{-}$and $K$ given in Appendix E, one can obtain the expression of $R$ in any representation (including reducible indecomposable ones). One can then check that the defining quadratic equations for the quantum plane and its Manin dual are respectively recovered by writing $A x x=0$ (respectively $S x x=0$ ). Here $S$ and $A$ are the two projectors entering the spectral decomposition of the numerical $\hat{R}=($ flip $) \cdot R$ matrix

$$
\begin{aligned}
\hat{R} & =q S-q^{-1} A \\
S+A & =\mathbb{1} \quad(\operatorname{Tr} S=3, \operatorname{Tr} A=1),
\end{aligned}
$$

$R$ being this time the numerical $R$-matrix in the fundamental representation. 


\section{References}

[1] D. V. Gluschenkov and A. V. Lyakhovskaya, Regular representation of the quantum Heisenberg double (q is a root of unity), Zapiski LOMI 215 (1994).

[2] L. Da̧browski, F. Nesti and P. Siniscalco, A finite quantum symmetry of $M(3, \mathbb{C})$, Int. J. Mod. Phys. A 13 (1998), 4147.

[3] A. Alekseev, D. Gluschenkov and A. Lyakhovskaya, Regular representation of the quantum group $S L_{q}(2)$ (q is a root of unity), St. Petersburg Math. J. 6 (1994), 88.

[4] R. Coquereaux, On the finite dimensional quantum group $M_{3} \oplus\left(M_{2 \mid 1}\left(\Lambda^{2}\right)\right)_{0}$, Lett. Math. Phys. 42 (1997), 309, hep-th/9610114.

[5] W. Pusz and S. L. Woronowicz, Twisted second quantization, Rep. Math. Phys. 27 (1989), 231.

[6] J. Wess and B. Zumino, Covariant differential calculus on the quantum hyperplane, Nucl. Phys. B (Proc. Suppl.) 18B (1990), 302.

[7] H. Weyl, The theory of groups and quantum mechanics (Dover Publications, 1931).

[8] Yu. I. Manin, Quantum groups and non commutative geometry, preprint Montreal Univ. CRM1561 (1988).

[9] O. Ogievetsky, Matrix structure of $S L_{q}(2)$ when $q$ is a root of unity, preprint CPT-96/P.3390, to appear.

[10] S. L. Woronowicz, Compact matrix pseudogroups, Comm. Math. Phys. 111 (1987), 613.

[11] R. Coquereaux and G. Schieber, Action of a finite quantum group on the algebra of $N \times N$ matrices, Proceedings of the Lodz Conference (1998).

[12] A. Connes, Gravity coupled with matter and the foundation of noncommutative geometry, Comm. Math. Phys. 182 (1996), 155.

[13] P. Humbert, Sur les fonctions de Bessel de troisième ordre, C. R. Acad. Sci. Paris (1930).

[14] R. Coquereaux, A. O. García and R. Trinchero, Finite dimensional quantum group covariant differential calculus on a complex matrix algebra, Phys. Lett. B 443 (1998), 221.

[15] R. Coquereaux, Noncommutative geometry and theoretical physics, J. Geom. Phys. 6 (1989), 425.

[16] A. Connes, Noncommutative geometry and reality, preprint IHES M/95/52.

[17] M. Dubois-Violette, K. Kerner and J. Madore, Classical bosons in a non-commutative geometry, Class. Quant. Grav. 6 (1989), 1709.

[18] R. Coquereaux, R. Haussling and F. Scheck, Int. J. Mod. Phys. A 7 (1992), 6555.

[19] H. Montani and R. Trinchero, Quantum mechanics over a q-deformed $(0+1)$-dimensional superspace, Int. J. Mod. Phys. A 13 (1998), 4173.

[20] Yu. I. Manin, Notes on quantum groups and quantum De Rahm complexes, Teor. i Matem. Fiz. Tom 92 N3 (1992), 425.

[21] O. Ogievetsky, Differential operators on quantum spaces for $G L_{q}(N)$ and $S O_{q}(N)$, Lett. Math. Phys. 24 (1992), 245.

[22] M. Rosso, Quantum groups at root of unity and tangle invariants, in Topological and Geometrical methods in Field Theory, J. Mickelsson and O. Pekonen (Eds.), World Scientific, 347 (1992). 